\title{
On Soret Convection in Binary and Pseudo-Binary Liquid Mixtures
}

\author{
Dissertation \\ zur Erlangung des mathematisch-naturwissenschaftlichen Doktorgrades \\ "Doctor rerum naturalium" \\ der Georg-August-Universität Göttingen
}

vorgelegt von

Kolja Kindler

aus Bad Gandersheim

Göttingen 2007 
D7

Referent: Prof. Dr. A. Tilgner

Korreferent: Prof. Dr. Dr. A. Dillmann

Tag der mündlichen Prüfung: 13. November 2007 



\section{Contents}

\begin{tabular}{lll}
\hline & Introduction & 1
\end{tabular}

2 Theoretical Background 3

2.1 Ravleigh-Bénard Convection in Binarv Fluids . . . . . . . . . . 3

2.1.1 The Oberbeck-Boussinesa Approximation . . . . . . . 3

2.1.2 Stability Analvsis and Pattern Formation . . . . . . . 5

2.1.3 Model Equations . . . . . . . . . . . . . . . . . 10

2.2 Phvsical Characterisation of Ternarv Microemulsions . . . . . . 11

2.2.1 Micro-Structure . . . . . . . . . . . . . . . . 12

2.2.2 Thermodvnamics of Microemulsions . . . . . . . . 13

2.2.3 Thermodvnamic Model Svstems . . . . . . . . . . . 16

2.3 Ravleigh-Bénard convection in Microemulsions . . . . . . . . . 17

2.3.1 Ravleigh-Bénard convection in Microemulsions . . . . . 17

2.3.2 The Colloidal Suspension Analogv . . . . . . . . . . . 19

2.3.3 Convection in the Vicinity of the Consolute Point . . . . 20

2.3.4 The Validity of the Boussinesq Approximation . . . . 22

3 Materials and Methods 25

3.1 Experimental Arrangement . . . . . . . . . . . . . . 25

3.1.1 The Visualisation Set-Up . . . . . . . . . . . . . 25

3.1.2 Set-Up for Velocity Measurements . . . . . . . . . . . . 27

3.2 Fluid Specimen . . . . . . . . . . . . . . . . . . . . . . . . 29

3.2.1 The Ethanol-Water Binarv Mixtures . . . . . . . . . . 29

3.2.2 The WDA Microemulsion . . . . . . . . . . . . 29

3.3 Methods of Investigation . . . . . . . . . . . . . . . . . . 30

3.3.1 Particle Image Velocimetrv . . . . . . . . . . . . . 30

3.3.2 Shadowgraph Visualisation . . . . . . . . . . . . . 34

3.3.3 Dielectric Relaxation Spectroscopy . . . . . . . . . . . 35

4 Results and Discussion 37

4.1 The Adaption of the $\mu \mathrm{PIV}$ Technique . . . . . . . . . . . 37

4.1.1 uPIV Data Evaluation . . . . . . . . . . . . . . 37

4.1 .2 The Impact of Tracer Particles . . . . . . . . . . . . . . 40

4.1.3 Convection in Pure $n$-Decand . . . . . . . . . . . . . 44

4.2 Binarv Fluid Convection . . . . . . . . . . . . . . . 46

4.2 .1 Stationarv Convection . . . . . . . . . . . . . . 46

4.2.2 Travelling Wave Convection . . . . . . . . . . . . . 55

4.2 .3 Confined Travelling Wave Convection . . . . . . . . . 59 
$4.3 \quad$ Velocity Measurements of Convection in a Microemulsion . . . 64

4.3.1 Convection in Microemulsions . . . . . . . . . . . . . 64

4.3.2 Effects of Impurities . . . . . . . . . . . . . . . . 68

4.4 Convective Pattern Formation in a Microemulsion . . . . . . 70

4.4.1 Off-Critical Convection in WDA . . . . . . . . . . . 70

4.4.2 Convection in the Vicinity of Phase Transition . . . . 75

4.4.3 The Effect of Incipient Decomposition . . . . . . . . . 79

5 Conclusions and Future Prospects 83

5.1 Conclusions . . . . . . . . . . . . . . . . . . . . 83

5.2 Future prospects . . . . . . . . . . . . . . 84

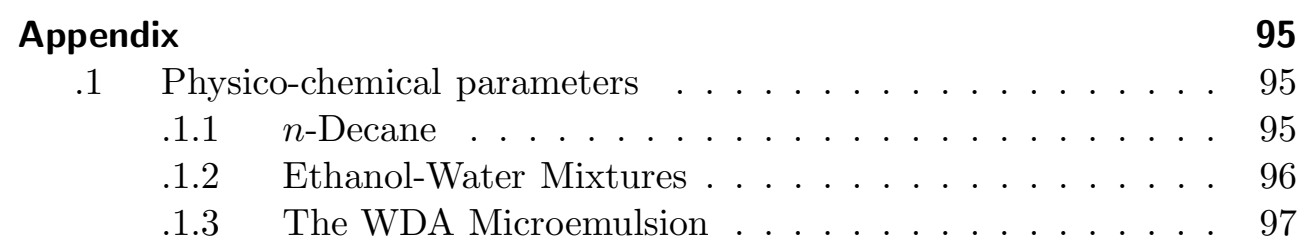




\section{List of Tables}

4.1 Composition and meso-structure parameters of the different WDA specimens; $D$ is estimated based on $R_{h}$ according to Eq. 2.26 . . . . . . . . . . . . . . . . . . . . 64

4.2 Thermodynamic parameter estimations based on the convective ampl tude in the Soret regime for the three different WDA specimen. . . . . . . . . . . . . . . 65

$.1 \quad$ Substance parameters of pure n-decane at $T=298 \mathrm{~K} . \ldots 95$

.2 Thermodvnamic properties of a $40 \% w t$ ethanol-water binary mixtures at $T=298 \mathrm{~K} \ldots \ldots \ldots \ldots$. . . . . . . 96

3 Thermodvnamic properties of a $25 \% w t$ ethanol-water binary mixtures at $T=298 \mathrm{~K} \ldots \ldots \ldots \ldots \ldots$. . . . . . 96

.4 Critical compositions and temperatures for the WDA svstem. 97

$.5 \quad$ Parameters and critical indices measured for the WDA svstem. 97

.6 Temperature dependent properties of the critical WDA svstem. 97 


\section{List of Figures}

2.1 Sketch of the Rayleigh-Bénard problem in a binary fluid. The fluid layer is confined between rigid top and bottom plates at $z / d= \pm 1 / 2$. The subscripts $t$ and $b$ refer to top and bottom, the reference values $\bar{T}$ and $\bar{c}$ are taken in the mid-plane of the geometry $(=0)$, and the acceleration due to gravity $\vec{g}$, points downwards. . . . . . . . . . . . . . . .

2.2 Sketch of the stability diagram for Soret convection in the plane of the reduced Rayleigh number $r$ and separation ratio $\psi$ according to 99. The full line indicates marginal stability of stationary onvectidn, the dashed line denotes oscillatory instability, and CT denotes the co-dimension two point at the intersection of both.

2.3 Sketch of the bifurcation diagram for convection with $\psi<0$ in the plane of the amplitude $A$ and reduced Rayleigh number $r$ after 72. The dashed line indicates the slope for a pure fluid, the b@ld solid line references travelling wave convection and the bold dashed line denotes stationary overturning convection. . .

2.4 Sketch of the $w / o$ micellar micro-structure of the WDA microemulsion. . . . . . . . . . . . . . . . 13

2.5 Sketch of the coexistence curve (solid line) of the WDA system and the spinodal (dashed). . . . . . . . . . . . . 14

3.1 Sketch of the Ravleigh-Bénard set-up and the optical arrangement of the shadowgraph system. . . . . . . . . . . . 26

3.2 Sketch of the Ravleigh-Bénard set-up utilised for direct velocity measurements. The outer enclosure of the heat bath ( $\mathrm{n}$ ot shown here) also consists of transparent polycarbonate (PC) . . . . 27

3.3 Calculated non-dimensional deviation from the linear temperature gradient at the lateral boundaries of the convection cell (in percent). The top and bottom boundaries are at $t / d= \pm 0.5$ and the lateral boundary is at $x / d=10$ (dotted line) . . . . . .

3.4 Sketch of the experimental arrangement for the handling of highly sensitive fluid mixtures. Pr\&ssurised nitrogen $\left(N_{2}\right)$ was used for initial filling of the convertion cell in order to avoid contact with atmospheric moisture. . . . . . . . . . . 29

3.5 Sketch of measurement arrangement for $\mu \mathrm{PIV}$ in the convection cell. . . . . . . . . . . . . . . . . . . 
3.6 Sketch of the coaxial reflexion cell (left) and principal representation of the reflexion factor measurement set-up (right), according to 108 . . . . . . . . . . . . . . . . . .

4.1 An example of the lateral velocity profile at $z / d=0$ : $\square$ denote ensemble dorrelation evaluation and $\bigcirc$ represent POD filtered evaluation. . . . . . . . . . . . . . . .

4.2 Two examples of the image density of a $48 \times 48$ pixel $\mu \mathrm{PIV}$ interrogation window pair $I_{a}$ and $t_{b}$ and the corresponding correlation plane $C$. The upper row is an ethanol-water mixture containing polystyrene spheres; the lower row depicts $n$-decane with cross-linked carboxyl tracers. . . . . . . . . . . . .

4.3 An example of the extraction of the maximum velocities in $x$ and $z$-direction. $\sup \{|w|\}$ denotes the supremum of $w$ along the vertical direction at fixed $x ; u$ is treated accordingly. The lower graph presents both $\sup \{|w|\}(0)$ and $w(x / d, 0)(\square)$ which nearly perfectly collapse onto the same curve. . . . . . . . . . .

4.4 The real $\varepsilon^{\prime}$ and imaginary part $\varepsilon^{\prime \prime}$ of the complex permittivity of three different specimens of WDA $(\omega=40.9, \eta=0.098$, $T=293 \mathrm{~K})$. The filled symbols indicate additional tracers; corresponding symbols refer to WDA specimens based on the same batch; the full lines represent the best fit to Eq. 4.4 . . . . . .

4.5 The maximum vertical velocity $w$ versus reduced Rayleigh number $r$ for decane and the theoretical curve according to Eq. 4.5 (full line) at $T_{t o p}=298 \mathrm{~K}$. The inset shows a magnification of the data close to $w=0$ where the abscissa depicts the temperature difference. The critical value is $\Delta T_{c}=0.596(5) \ldots \ldots$. .

4.6 The maximum vertical and horizontal velocities $w(0)$. $u(\square)$ versus reduced Rayleigh number $r$ for ethanol - water m ixture with $\psi=0.23, L \simeq 0.0045$ at $T_{t}=298 \mathrm{~K}$. The inset is a magnification of the $u$-component close to $r=0$. The dashed lines indicate structural transitions from rolls to cross-rolls at $r=0.91(1)$ and cross-rolls to the large-scale structure at $r=0.56(2) \ldots$. .

4.7 The lateral Fourier mode amplitudes in the transition region between the Rayleigh and Soret regime; the error bars indicate the uncertainty of the approximation. . . . . . . . . .

4.8 The lateral $u$ (dash-dotted) $w$-profiles (full line) approximated by the first two Fourier modes $\}=1.11(1)$ (top), $r=1.00(1)$ (centre), and $r=0.92(1)$ (bottom). . . . . . . . . . . 50

4.9 The eigenvalues $\lambda$ of the first three POD mode versus $r$ in the

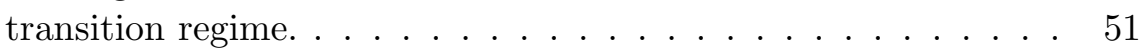

4.10 The velocitv field representation of the eigenmodes one to three of the decomposition at $r=1.16(1) \ldots \ldots \ldots \ldots$ 
4.11 Comparison of the velocity amplitude and the model of Eq.4.7. The inset clarifies the mismatch of the model and the experimental data for the large-scale structure and depicts a representative error bar. . . . . . . . . . . . 54

4.12 The eigenvalues $\lambda_{i}$ of the first three POD mode versus $r$ of TW convection in a $25 \% w t$ ethanol-water mixture with $\psi=-0.087$.

4.13 The velocitv field representation of the first and the second POD eigenmode of the flow at $r=1.08(2) \ldots \ldots \ldots \ldots$

4.14 The first and second Fourier mode amplitude of $u$ and $w$ in the TW state of the 25\% owt ethanol-water mixture with $\psi=-0.087$, $\sigma \simeq 18$, and $L \simeq 0.0061$; the error bars denote the uncertainty of the approximation. . . . . . . . . . . . . 58

4.15 The frequency of the TW $\omega$ versus $r$. . . . . . . . . . 59

4.16 The flow field representation of first and second POD mode of the confined TW state at $r=1.03(4)$ (top and centre) an the time evolution coefficients $\zeta_{i}$ for the two modes (bottom). . . .

Time series of the two-mode approximation of the velocity field comprising the boundary of a confined TW state at $r=1.03(4)$ (from left to right and top to bottom); the time separation of the fields is $0.24 \tau_{v}$. The velocity fields cover the layer depth $d$ and the lateral extent is $2 d$. . . . . . . . . . . . . .

4.18 The maximum vertical velocity, $w$, versus lateral position, $x$, extracted at $z / d=0$ from the two-mode approximation depicted in Fig. 4.16. The solid line represents exponential decay of the form $w(0, x) \sim e^{-1.6 x} \ldots \ldots \ldots \ldots \ldots$

$4.19 u$ and $w$ versus $r$ measured in the water-less micellar solution (specimen 1); the solid line denotes the pure fluid amplitude. . 66

4.20 The maximum of the $u$-component representing the convection amplitude versus $r$ of the three different WDA specimens in comparison to the theoretical pure fluid solution. . . . . . . . .

4.21 The first observable, spoke-like structures in the Soret regime of WDA $r=0.52(1)$ (left) and the evolving square-like pattern at $r=0.89(1)$ (right) in the off-critical region. . . . . . . . . .

4.22 Square-like structures in the late stage of the Soret regime $r=0.92(1)$ (left) and $r=0.95(1)$ (right). . . . . . . . . .

4.23 An example of competing square and roll structures in the transition to the Rayleigh regime $r=1.02(1) \ldots \ldots \ldots$. . . . .

4.24 Small-scale structures as the initial pattern of the Soret mode and its struptural development via knot-bifurcations, disordered small-scale structures and large-scale spoke pattern with increasing $r=0.32$ (1) to $r=0.71$ (1) (from left to right and top to bottom). . . . . . . . . . . . . 76

4.25 Disordered convective pattern in the Soret regime approaching the phase transition $r=0.32$ (1) (left) and $r=0.74$ (1) (right). . . 
4.26 Superposition of the roll pattern at $r=1.03(1)$ by additional branches close to $T_{c} \ldots \ldots \ldots \ldots \ldots \ldots$

4.27 Plumes of separated concentration droplets locally rising from the lower boundary layer superimposing the polygonal pattern at $\bar{T} \simeq T_{c}$ and $r=0.83(1)$ (left) and TW structures of aligned droplet streaks cldse to the onset of spinodal decomposition at $r=0.89(1)$ (right). . . . . . . . . . . . . .

4.28 An earlv (left. $r=0.54(1)$ ) and a very late stage pattern of incipient spinodal decomposition (right, $r=0.79(1)$ ) at $\bar{T}>T_{c}$. The ripply substructure on the left image is due to a sub-layer of separated fluid. . . . . . . . . . . . . . . 


\section{Nomenclature}

\section{Latin Symbols}

$\begin{array}{ll}a & \text { core radius } \\ A & \text { amplitude } \\ B & \text { Biot number } \\ \tilde{c} & \text { concentration deviation from the reference } \\ c & \text { concentration of the minor component } \\ C & \text { correlation matrix } \\ C_{p} & \text { isobaric specific heat } \\ C_{v} & \text { isochoric specific heat } \\ d & \text { vertical length scale } \\ D & \text { mass diffusion coefficient } \\ D_{T} & \text { thermo-diffusion coefficient } \\ E & \text { energy } \\ f & \text { frequency } \\ \vec{g} & \text { gravitational acceleration } \\ h & \text { heat transfer } \\ i & \text { imaginary unit } \\ I & \text { intensity } \\ j_{c} & \text { concentration current } \\ k & \text { wave number } \\ k_{B} & \text { Boltzmann constant } \\ k_{T} & \text { thermo-diffusion ratio } \\ K & \text { combined deviation field } \\ l & \text { characteristic length } \\ L & \text { Lewis number } \\ n & \text { refractive index } \\ N & \text { Nusselt number } \\ p & \text { pressure } \\ p_{i} & \text { POD eigenmodes } \\ P & \text { polarisation } \\ q & \text { heat flux } \\ Q & \text { deviation parameter } \\ r & \text { reduced Rayleigh number } \\ R & \text { Rayleigh number } \\ R_{m} & \text { micellar radius } \\ R_{h} & \text { hydrodynamic radius } \\ t & \text { time } \\ T & \text { temperature } \\ & \end{array}$




$\begin{array}{ll}\bar{T} & \text { reference temperature } \\ \vec{u}=(u, v, w) & \text { local velocity } \\ v=\left(u^{2}+w^{2}\right) & \text { mean velocity in } x, z \text {-plane } \\ V & \text { volume } \\ \vec{x}=(x, y, z) & \text { spatial coordinates } \\ X & \text { unspecified variable } \\ z & \text { critical index of the viscosity }\end{array}$

\section{Greek Symbols}

$\alpha$

$\alpha, \gamma, \nu, \delta$

$\beta$

$\Gamma$

$\delta_{c}$

$\Delta$

$\epsilon$

$\varepsilon$

$\zeta_{i}$

$\eta$

$\Theta$

$\kappa$

$\lambda$

$\lambda_{c o}$

$\lambda_{i}$

$\mu$

$\nu$

$\xi$

$\rho$

$\sigma$

$\tau$

$\phi$

$\varphi$

$\chi$

$\psi$

$\omega$

$\omega_{H}$

thermal expansion

critical indices

concentration expansion

wave length

concentration boundary layer thickness

vertical difference

reduced distance to $T_{c}$

permittivity

time evolution coefficient of the POD

volume fraction

temperature deviation

thermal diffusivity, electric conductivity

thermal conductivity

growth rate

eigenvalues of the POD

dynamic viscosity, chemical potential

kinematic viscosity

correlation length

density

Prandtl number

relaxation time

interaction potential

phase angle

susceptibility

separation ratio

molar ratio

Hopf frequency

\section{Indices}

linear reference profile

bottom 


$\begin{array}{ll}b c & \text { boundary condition } \\ c & \text { critical } \\ f & \text { fluid } \\ h & \text { horizontal } \\ p & \text { pure fluid reference } \\ t & \text { top } \\ v & \text { vertical }\end{array}$

\section{Miscellaneous Symbols}

$\begin{array}{ll}\rightleftarrows & \text { aspect ratio } \\ \mathscr{L} & \text { Laplace transformation } \\ \partial_{X} & \text { partial derivative with respect } X \\ \nabla & \text { differential operator }\end{array}$

\section{Abbreviations}

AHS

DRS

$\mu \mathrm{PIV}$

POD

SOC

TW

$w t$ adhesive hard-sphere model dielectric relaxation spectroscopy micro Particle Image Velocimetry Proper Orthogonal Decomposition stationary overturning convection travelling wave weight 


\section{Introduction}

Thermal convection, i.e. buoyancy induced fluid flow in response to thermal excitation, is a frequently occurring phenomenon in nature 94. The investigation of convection in thin fluid layers is a classical problem of hydrodynamics and Rayleigh-Bénard convection has a long-ranging history in the investigation of instabilities and pattern formation in systems far from equilibrium [26]. A particularly rich bifurcation and pattern formation behaviour is found in convection in binary fluids, i.e. fluid mixtures of two different species since the concentration and temperature fields are coupled by the Soret effect [117. In response to a temperature gradient concentration fluxes are generated which either amplify or compensate the density variations within the fluid layer, giving rise to peculiar spatio-temporal behaviour [99].

The concept of binary fluid convection has been demonstrated to apply for mixtures of species with large differences in size, as for example nano-particle suspensions [53]. These fluids are commonly referred to as pseudo-binary mixtures which also includes complex mixtures forming meso-scale structures, i.e. structures intermediate to molecular and macroscopic, hydrodynamic length scales 37. A representative of such complex fluids are microemulsions, mixtures of water, oil, and a surface-active-agent (commonly abbreviated as surfactant). The surfactant molecules occupy the interface between the oil and the water forming nano-size, micellar-like droplets of the minor phase [102. These mixtures have numerous applications as for example for nano-particle syntheses [82 and, more recently, as drug-delivery media in pharmacy [75].

The present investigation mainly focusing on convection in a microemulsion is essentially motivated by the fact that these systems exhibit a thermodynamic critical point in terms of a liquid-liquid phase transition. This consolute point can be found close to room temperature at ambient pressure which is experimentally easily accessible. In the vicinity of the consolute point (the critical region) the fluid is distinguished by increasingly strong density or concentration fluctuations causing many thermodynamic and transport parameters to diverge towards the critical point. As the Soret effect can be anticipated to be greatly amplified in the critical region, very strong concentration driven convection can be expected in response to particularly small thermal stresses potentially leading to turbulence.

Rayleigh-Bénard convection in the vicinity of a liquid-vapour critical point of pure fluids has been theoretically and experimentally investigated and it is understood that the critical region mainly constitutes a transfer to compressible convection [39]. However, the behaviour at a consolute point of a mixture is largely different since the impact of the corresponding effect, i.e. the osmotic 
compressibility and its concurrence with the Soret effect is a priori unclear.

It will be shown that microemulsion exhibits a positive Soret effect, i.e. thermal and solutal effects contribute to the destabilisation of the fluid layer leading to almost purely concentration driven convection close to onset which will be referred to as the Soret regime. In this regime very small convective amplitudes inhibit classical experimental methods such as heat transfer measurements or shadowgraph visualisation. Therefore, the micro-Particle Image Velocimetry $(\mu \mathrm{PIV})$ technique has been adapted to resolve flow velocities of the order of $\mathrm{O}(\mu \mathrm{m} / \mathrm{s})$.

In a first instance the Soret regime of a molecular binary mixtures will be discussed in detail ranging from the onset of convection to the transition to purely thermally driven convection. In connection with an excursion on negative Soret coupling the competition of diffusion transport and advection in presence of convective motion will be elucidated. In comparison to that, it will be seen that convection in pseudo-binary microemulsions is determined by a decoupling of the temperature and concentration fields. Due to the extremely small diffusion transport of the large micellar structures, as compared to molecules, the concentration variations within the fluid are "frozen" on experimentally realisable time scales.

Therefore, the concentration stratification of the fluid layer in response to a thermal gradient is restricted to an extremely slowly growing concentration boundary layer at the heated boundary. At the same time the Soret effect is considerably stronger than in molecular mixtures leading to a spontaneous onset of convective motion. Furthermore, approaching the critical region, the particular steep gradient of the concentration boundary layer leads to localised phase separation which transfers the system into a two-phase state well before the critical point is reached. In the vicinity of the critical point in presence of large enough convective motion to prevent localised phase separation, the critical enhancement of the separation ratio will be shown to increasingly amplify convection.

The thesis is structured as follows. In the second chapter the theoretical background of the experiments is presented covering the Boussinesq approximation for binary fluid convection, a brief introduction to microemulsions, and a literature review on convection in microemulsions. Subsequently, the experimental arrangement and methods of investigation will be introduced and the adaption of the $\mu \mathrm{PIV}$ technique will be described. The discussion of the experimental results will be subdivided into two main parts. Initially, a detailed investigations of Soret convection in molecular binary mixtures will be presented and discussed. In a second stage Rayleigh-Bénard convection in a microemulsion will be addresses based on both direct velocity amplitude measurements and pattern visualisation in the critical region. Finally, the discussion is closed by the conclusions and prospections of future work. 


\section{Theoretical Background}

In this chapter Rayleigh-Bénard convection in a (pseudo-) binary fluid will be formalised based on the classical Boussinesq formulation, and a summary of the microemulsion theory will be presented sketching the microscopic structure and the thermodynamics of the system. Subsequently, the literature on convection in microemulsions will be reviewed, the effects of approaching the phase transition region will be addressed, and the limitations of the Boussinesq approximation will briefly be outlined.

\subsection{Rayleigh-Bénard Convection in Binary Fluids}

\subsubsection{The Oberbeck-Boussinesq Approximation}

Consider a horizontal binary fluid layer of infinite lateral extend, confined between two perfectly thermally conducting, rigid, impermeable boundaries at $z= \pm d / 2$ subjected to a vertical temperature difference $\Delta T=T_{b}-T_{t}$ by heating from below (the subscripts $t$ and $b$ denote top and bottom, cp. Fig. 2.1). The vertical temperature gradient $\Delta T / d$ causes buoyancy resulting from the variation of the density with temperature which can be written by a first order Taylor expansion of the form

$$
\rho=\bar{\rho}(1-\alpha(T-\bar{T})-\beta(c-\bar{c})),
$$

where $c$ is the concentration of the minor component. The reference states $\bar{\rho}=\left(\rho_{b}+\rho_{t}\right) / 2, \bar{T}=\left(T_{b}+T_{t}\right) / 2$, and $\bar{c}=\left(c_{b}+c_{t}\right) / 2$ are taken at mid-height of the fluid layer and

$$
\alpha=\frac{1}{\bar{\rho}} \frac{\partial \rho}{\partial T} \quad \text { and } \quad \beta=\frac{1}{\bar{\rho}} \frac{\partial \rho}{\partial c}
$$

are the thermal and concentration expansion, respectively.

In general, a temperature gradient induces a mass flux in a multi-component mixture which is called thermal diffusion or the Ludwig-Soret effect. Thus the concentration current in response to $\Delta T / d$ takes the form 74 ]

$$
\overrightarrow{j_{c}}=-D(\nabla c+\psi \nabla T)+\vec{u} c,
$$

with the diffusion coefficient $D$, the local velocity $\vec{u}$, and the separation ratio

$$
\psi=-\frac{\beta}{\alpha} \frac{k_{T}}{T} \bar{c}(1-\bar{c})
$$

The parameter $k_{T}=D_{T} / D$ is the thermo-diffusion ratio with the thermal diffusion coefficient $D_{T}$. The separation ratio $\psi$ defines the coupling of the 


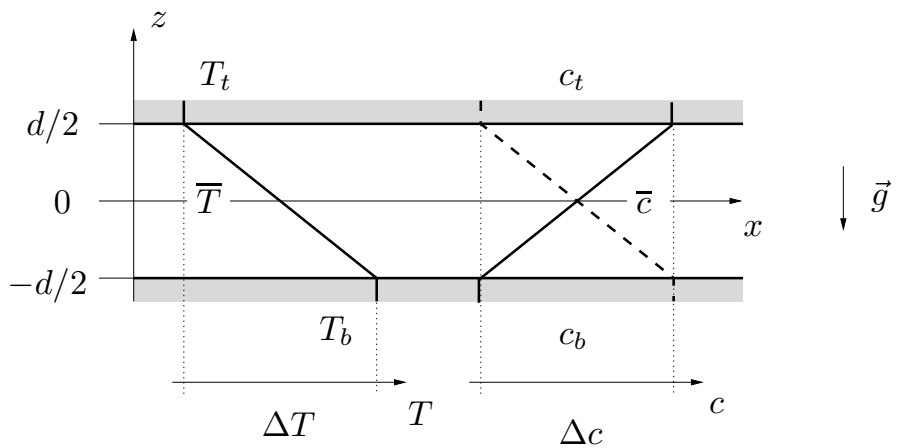

Figure 2.1: Sketch of the Rayleigh-Bénard problem in a binary fluid. The fluid layer is confined between rigid top and bottom plates at $z / d=$ $\pm 1 / 2$. The subscripts $t$ and $b$ refer to top and bottom, the reference values $\bar{T}$ and $\bar{c}$ are taken in the mid-plane of the geometry $(z=0)$, and the acceleration due to gravity $\vec{g}$, points downwards.

temperature and concentration gradient. Therefore, the linear temperature profile $T_{0}(z)$ also implies a linear concentration profile $c_{0}$.

$$
T_{0}(z)=\bar{T}+\Delta T(z-d / 2) \quad c_{0}(z)=\bar{c}+\psi \Delta T(z-d / 2)
$$

The sign of $\psi$ depends on the particular mixture under consideration, i.e. the coupling of concentration and temperature gradients can be parallel or antiparallel (see Fig. 2.11). The contribution of the concentration gradient to the heat current, the Dufour effect, is negligible for fluids and shall be disregarded here.

The Boussinesq approximation is based on the momentum, mass, concentration, and energy conservation in a binary mixture subjected to a temperature gradient fixing the fluid parameters such as the isobaric specific heat $C_{p}$, the thermal diffusivity $\kappa$, the dynamic viscosity $\nu$, and the thermo-diffusion ratio $k_{T}$ to the reference state at $\bar{T}$. The gravity $\vec{g}$ is constant throughout the fluid layer, pointing downwards, and internal heat generation is assumed to be negligible. Furthermore, the density variations are only taken into account in the buoyancy term of the Navier-Stokes equation.

The variables are rescaled using the vertical spacing $d$, the (vertical) thermal diffusion time $\tau_{v}=d^{2} / \kappa$, the non-dimensional temperature $(\kappa \nu) /\left(g \alpha d^{3}\right)$, and $\left(\nu \kappa\left(k_{T} / \bar{T}\right) \bar{c}(1-\bar{c})\right) /\left(\alpha g d^{3}\right)$. The linear, conductive profiles $T_{0}$ and $c_{0}$ are subtracted from the concentration $\tilde{c}=c(z)-c_{0}(z)$ and the temperature, $\Theta=T(z)-T_{0}(z)$ profile and the auxiliary combined field $K=\tilde{c}+\psi \Theta$ is in- 


\subsection{Rayleigh-Bénard Convection in Binary Fluids}

troduced. The field equations for binary fluids thus read [26]:

$$
\begin{array}{r}
{[l] \sigma^{-1}\left(\partial_{t}+\vec{u} \cdot \nabla\right) \vec{u}=-\nabla p+(\Theta(1+\psi)-K)+\nabla^{2} \vec{u}} \\
\left(\partial_{t}+\vec{u} \cdot \nabla\right) \Theta=R \cdot w+\nabla^{2} \Theta \\
\left(\partial_{t}+\vec{u} \cdot \nabla\right) K=L \nabla^{2} K+\psi \nabla^{2} \Theta \\
\nabla \vec{u}=0 .
\end{array}
$$

The above given equations Eq. 2.6 to 2.9 have to be supplemented with realistic "rigid-rigid" boundary conditions

$$
\vec{u}=\Theta=\frac{\partial \zeta}{\partial z}=0, \quad \text { at } z= \pm \frac{1}{2}
$$

Thereby, Rayleigh-Bénard convection in a binary fluid is characterised by four dimensionless quantities. The fluid properties are specified by the ratio of the diffusivity of momentum and heat given as the Prandtl number $\sigma=\nu / \kappa$. Additionally, the Lewis number $L=D / \kappa$ defines the ratio of mass diffusion and thermal diffusivity. For the Lewis number it is convenient to think of it as the ratio of the thermal and diffusive relaxation times, i.e. the times required to reach a static concentration and temperature profile across the fluid layer after changing the temperature gradient. The vertical relaxation times read $\tau_{D}=d^{2} / D$ and $\tau_{\kappa}=d^{2} / \kappa$, respectively. In this manner $L$ provides a measure for the delay of e.g. the concentration response with respect to the thermal gradient as in molecular binary mixtures the Lewis number is commonly of the order of $L=\mathrm{O}\left(10^{-2}\right)$. The impact of $L$ will be of particular importance in this work because the largeness of the meso-structure in microemulsions leads to a very small mass diffusion yielding Lewis numbers of $L=\mathrm{O}\left(10^{-5}\right)$ which effectively rules out relaxation of the concentration (cp. Sec. 2.3.2). Analogously, the Prandtl number can be regarded as the ratio of the thermal and viscous relaxation times.

Furthermore, the Rayleigh number representing the ratio of buoyancy to viscous forces

$$
R=\frac{\alpha g \Delta T d^{3}}{\nu \kappa}
$$

defines the external stress applied to the system, which is the experimental control parameter. The fourth parameter is the separation ratio $\psi$ providing the coupling of temperature and concentration gradients. The sign of $\psi$ indicates if the Soret effect amplifies or suppresses the density stratification due to thermal expansion.

\subsubsection{Stability Analysis and Pattern Formation}

The coupling of the thermal and concentration expansion within the fluid layer gives rise to two qualitatively different situations depending on the sign of the separation ratio $\psi$. In the limit of a negligibly small thermal stratification 
with respect to concentration in terms of large $\psi>0$, convection is purely driven by the Soret effect, i.e. by a destabilising concentration gradient. In the limit of negligible solutal effects $(\psi=0)$ Eq. 2.6 to 2.9] reduce to the wellknown Boussinesq approximation for a pure or one-component fluid. In the pure fluid case, the system exhibits a forward bifurcation from the conductive state to stationary, parallel horizontal rolls as the initial instability [16]. The critical Rayleigh number for the onset of convection in a one-component fluid is $R_{c}^{p}=1707.762$ and the critical wave number of the roll structures is $k_{c}^{p}=3.117$, where the superscript $p$ indicates the pure fluid case and the subscript $c$ denotes the critical value [20]. The wave number is $k=(2 \pi d) / \Gamma$, where $\Gamma$ is the wave length of the roll pattern. In the remainder $R_{c}^{p}$ will be used to scale the Rayleigh number, $r=R / R_{c}^{p}$.

\section{Positive Separation Ratio}

In the case of the "normal" or positive Soret effect $(\psi>0)$, both the concentration and the temperature gradient contribute to the destabilisation of the fluid layer. Thus, the onset of convection occurs at smaller Rayleigh numbers as compared to pure fluids $r<1$ (see Fig. 2.2). In agreement with the linear stability analysis of the Boussinesq approximation the initial bifurcation is observed to lead to stationary convection 61. As an effect of the impermeability of the boundaries to concentration fluxes, the convective pattern consists of two perpendicular horizontal roll modes with equal amplitudes forming square structures. Furthermore, the critical wave number at the onset of convection is reduced with respect to the pure fluid case $k_{c}<k_{c}^{p}$ [87]. Moreover $k_{c}$ is found to decrease with increasing separation ratio and to become vanishingly small for finite $\psi$ when thermal effects can be neglected with respect to the concentration. For the special case of a fluid layer of infinite lateral extend and $k_{c}=0$ the critical Rayleigh number can be derived explicitly as [43, 44]

$$
R_{c}=720 \frac{L}{\psi} .
$$

The lateral confinement in an experiment limits the critical wave number to finite values $k_{c}=\pi / Æ$ with the aspect ratio $Æ=l / d$, where $l$ is the longest horizontal extend of the fluid layer.

In order to elucidate the physics of convection at a sufficiently large separation ratio $\psi$ for solutal and thermal contributions to decouple, the thermal insulator analogy may be used [89]. To this end, the impermeability of the boundaries for concentration fluxes is identified with (perfectly) insulating boundaries for the temperature field. In a more rigorous formulation, the thermal conductivity at the boundaries is formalised by the Biot number which is the ratio of the boundaries' thermal conductivity (subscript $b c$ ) to that of the fluid (subscript $f$ ), $B=\lambda_{b c} / \lambda_{f}$ [54]. If the boundaries were thermally insulating $\left(\lambda_{b c}=0\right.$ and thus $B=0$ ) no heat could be transferred through the 


\subsection{Rayleigh-Bénard Convection in Binary Fluids}

boundary and initially warmer and ascended fluid would not sink again. If, on the other hand, the boundaries are poorly conducting $(0<B \leq 1)$, the heat transfer into and out off the fluid requires an extended residence time of the fluid in contact to the boundaries leading to larger scale convective structures corresponding to smaller wave numbers $k$.

The stability analysis of the Rayleigh-Bénard problem in association with poorly conducting boundaries predicts the horizontal roll solution to be unstable to a perpendicular roll mode, forming a square pattern [54]. Furthermore, in this formalism, a transition from regular roll structures to two perpendicular roll modes is found at $B=\mathrm{O}(1)$ for the Prandtl numbers relevant to this work. Additionally, the critical Rayleigh number reduces from $R_{c}=1708$ and $k_{c}=3.117$ at $B \geq 10^{3}$ to $R_{c}=720$ and $k_{c}=0.6$ for $B=10^{-3}$.

Thus, the pattern formation and wave number selection of a binary mixture with positive Soret effect can be thought of as an effect of the boundary condition virtually changing. At a constant separation ratio $\psi$ the Soret regime, the regime of concentration dominated convection, can be identify with $B$ close to zero. Correspondingly, there is no exchange of concentration at the boundary and the fluid is convected to infinity which is identified with the large-scale structure (region a in Fig. 2.2). In this situation the wave number is not selected by the linear instability of the conductive state, but it is determined by the the convection cell dimension instead.

Increasing the thermal stress $(r)$ the heat exchange at the boundaries gains importance with respect to concentration and the wave number increases. The large-scale structure evolves into disordered and later on ordered square structures with increasing $k$ as $B$ virtually rises (b and c in Fig. 2.2). In agreement with that, square pattern are found to be stable for not to small $\psi$ depending on the bulk fluid properties, represented by the Prandtl number $\sigma$ [23]. In this regime of square structures, a linear stability analysis shows the fastest growing wave number to remarkably increase with increasing $r$ which is an explanation for the large-scale structure close to onset to evolve into smaller scales [23]. However, the wave number selection in this regime remains not fully understood yet.

The square-shaped structures persist approximately until $r=1$ where the conductive state in a pure fluid with equal thermodynamic properties undergoes a bifurcation to rolls. At this point, the thermal expansion dominates leading to larger amplitude convection and advecting concentration differences $(B \gg 1)$. Past the transition from the Soret regime to thermally driven convection, concentration effects are repressed into the thin boundary layers and convection becomes indistinguishable from the pure fluid case. It will be referred to the ordered roll structure at $r \geq 1$ as the Rayleigh regime drawing a distinction to the Soret regime at $r<1$.

In addition to the stationary square and roll modes, the nonlinear stability analysis predicts oscillatory cross-rolls in the transition regime for small Lewis numbers $L[53$. The cross-roll structure is a set of two perpendicular horizon- 


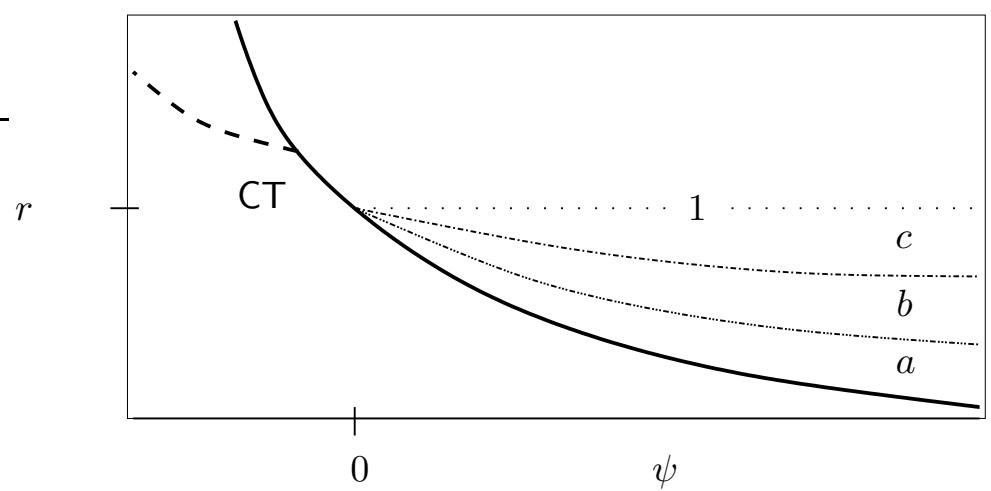

Figure 2.2: Sketch of the stability diagram for Soret convection in the plane of the reduced Rayleigh number $r$ and separation ratio $\psi$ according to 99. The full line indicates marginal stability of stationary convection, the dashed line denotes oscillatory instability, and CT denotes the co-dimension two point at the intersection of both.

tal roll modes which differ in amplitude while for the oscillatory cross-rolls the amplitudes oscillate in counter-phase. The latter was experimentally observed in an ethanol-water mixture by Moses \& STEINBerg [87] who have reported alternating waves penetrating the cell from the boundaries. Since the oscillations were observed in large aspect ratio cells of rectangular and cylindrical geometry, the oscillatory state is understood to be intrinsic to the competition of roll and square modes.

\section{Negative Separation Ratio}

If $\psi<0$, the Soret effect hinders the instability of the fluid layer. The thermal gradient induces an stabilising concentration gradient and the onset of convection is shifted to larger RAyleigh numbers $r>1$. When fluid initially rises, the vertical concentration stratification is reduced, resulting in an amplification of convective motion. Thus the concurrence of the concentration gradient with destabilising thermal expansion causes an inverted Hopf bifurcation to oscillatory convection with an exponentially growing amplitude which saturates in a state of nonlinear travelling waves (TW) as the initial instability [99] (Fig. 2.3). The onset itself is discontinuous and hysteretic which reflects the nonlinear feedback mechanism. With increasing $r$, the conductive state loses stability at $r_{c o}$ (cp. Fig. 2.3) giving raise to two counter-propagating travelling wave modes with exponentially growing amplitude. The linear stability analysis of the Boussinesq approximation predicts the onset of TW for free-slip 


\subsection{Rayleigh-Bénard Convection in Binary Fluids}

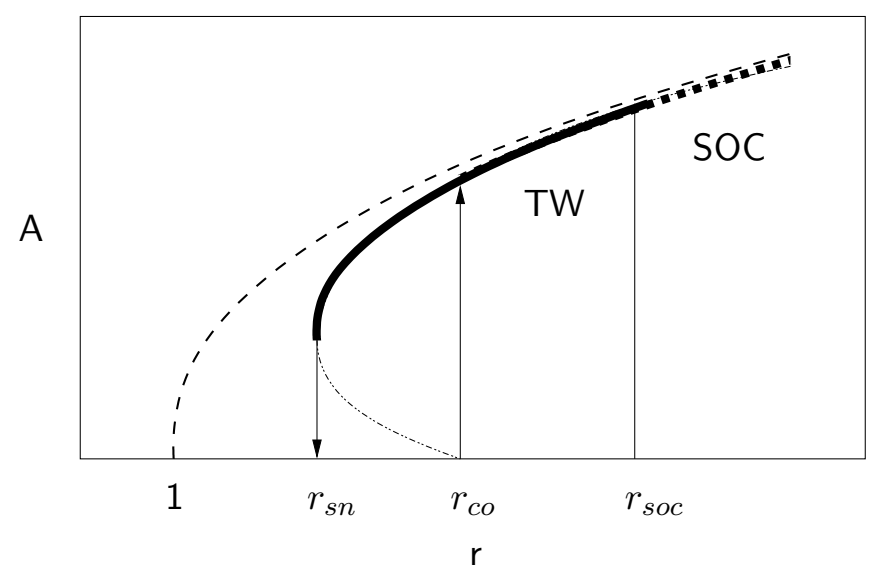

Figure 2.3: Sketch of the bifurcation diagram for convection with $\psi<0$ in the plane of the amplitude $A$ and reduced Rayleigh number $r$ after [72]. The dashed line indicates the slope for a pure fluid, the bold solid line references travelling wave convection and the bold dashed line denotes stationary overturning convection.

boundary conditions at

$$
r_{c o}=1-\frac{\psi}{(1+\psi+1 / \sigma)},
$$

which has to be corrected for the realistic no-slip conditions by the addition of $5 \%$ in the last term 62. Generally, the oscillatory instability threshold depends on $\psi$ and the intersection with the Hopf neutral curve and the steady state neutral curve is called the co-dimension two point (indicated as CT in Fig. 2.2) which will not be considered here (see e.g. [27] for further information).

The frequency of the oscillation $\omega$ is related to the strength of the Soret coupling $(\psi)$ and the instability occurs at finite wave numbers $k_{c}$ close to the pure fluid value $k_{c}^{p}$ [61. The initial frequency of the TW at onset (the Hopf frequency) varies as

$$
\omega_{H} \simeq-449 \frac{\psi}{(1+\psi+1 / \sigma)}
$$

according to linear stability analysis 9 . The competition of the two growing TW modes, including reflection from the lateral boundaries in finite geometries, saturates in a single TW mode with a frequency of approximately $1 / 10 \omega_{H}[$.

Lowering $r$ from $r \geq r_{c} O$ the TW state reduces in amplitude and the frequency increases reaching to approximately $1 / 3 \omega_{H}$ at $r_{s n}$. At a further reduction of $r$ the systems undergoes transition to conduction. Increasing $r$ 
above $r_{c o}$ on the other hand leads to a continuous transition from TW to a stationary roll mode, which for historical reason is referred to as stationary overturning convection (SOC) at $r_{S O C}$ (Fig. [2.3) 9, 72].

In addition to the homogeneous TW states, in the region above the onset of convection and the transition to stationary rolls, $r_{c o}<r<r_{S O C}$, spatially confined TW states can occur in finite geometries. These patterns remain after a TW state initially present over the whole fluid layer decays in certain parts of the convection cell. The nature of these confined TW's can be understood as a result of reflected counter-propagating TW in finite geometries and back-flow due to mass conservation [86].

\subsubsection{Model Equations}

In order to develop an idea of the velocity field in two-dimensional convection it is common practice to refer to model equations for pure fluid convection, derive the velocity field, and to regard the pure fluid case as a reference. Close to the bifurcation from the conduction to convection an amplitude equation can be derived from the Oberbeck-Boussinesq approximation which takes the form of a time-dependent Ginzburg-Landau form 24 .

$$
\tau_{0} \partial_{t} A=(r-1) A+\xi_{0}^{2}\left(\partial_{x}-\left(i / 2 k_{c}\right) \partial_{y}^{2}\right)^{2} A-g_{0}|A|^{2} A .
$$

In genera, the $A$ is a complex amplitude and the parameters, $\tau_{0}, \xi_{0}$, and $g_{0}$ are real for pure fluids. Furthermore, $i$ is the imaginary unit, $k_{c}$ is the critical wave number and $\partial_{t}, \partial_{x}$, and $\partial_{y}$ denote partial derivatives with respect to the two horizontal directions $x, y$, and time $t$. The amplitude equation primarily provides a basis for the investigation of the stability of the Boussinesq approximation and the pattern formation close to the bifurcation. Furthermore, the saturation amplitude, the long-time limiting value of $A$ at a particular Rayleigh number $r$ yields quantitative predictions for the maximum velocity amplitude, critical wave number, and heat transfer 93 .

A further approach to the description of the spatially periodic fields, of the velocity $\vec{u}$, temperature $\Theta$, and concentration $c$ is the representation by a truncated set of Galerkin modes. In the two-dimensional, vertical cross-section of the fluid layer ( $x, z$-plane) the expansion is of the form

$$
X(x, z)=\sum_{m} \sum_{n} X_{m n} \cos (m k x) f_{n}(z),
$$

where $X$ is a substitute for the above given field variables. The sum is generally truncated and the $f_{n}$ are convenient functions to satisfy the boundary conditions. The balance equations of the Boussinesq approximation (Eq. 2.6 - 2.9) are than projected onto the basis of the expansion and the parameters $X_{m n}(R, \sigma)$ are determined by solving the resulting equations [94, 16]. This approximation is widely used in the numerical treatment of Rayleigh-Bénard 


\subsection{Physical Characterisation of Ternary Microemulsions}

convection, where the $c, \Theta$, and $\vec{u}$ fields are approximated to a varying degree [50].

Accordingly, the velocity in the vertical cross-section of the stationary roll mode close to $R_{c}^{p}$ can be expanded into a Fourier series according to

$$
u(x, z, t)=u_{0}(z)+\sum_{n} u_{n}(z, t) e^{i n k x} .
$$

An analogous expression applies for $w$. For pure fluids the field is found to be accurately approximated by two modes [20], which, more generally, applies for stationary roll modes and travelling waves $[9$. The time dependence of the Fourier amplitudes

$$
u_{n}(z, t)=\left|u_{n}(z)\right| e^{-i \varphi_{n} t}
$$

includes travelling waves with a linear varying phase $\varphi_{n}$. Normand \& PomMEAU 94 have derived $u_{1,2}$ and $w_{1,2}$ explicitly for pure fluids based on a perturbative approach using a Landau-Hopf scheme in the limit of an infinite Prandtl number $\sigma=\infty$ which will be used as the pure fluid reference to the measured values. These authors arrive at an expansion of velocity components with respect to the distance from $R_{c}$ of the form

$$
u(r, z)=\sum_{n} u_{n}(z)(r-1)^{\frac{1}{2} n}
$$

where the $u_{n}$ are the $z$-dependent Fourier amplitudes. The maximum velocity amplitudes of the individual modes have been shown to be in excellent agreement with direct velocity amplitude measurements in silicone oil $(\sigma=\mathrm{O}(100))[12,33$ and stationary convection in water-isopropanol mixtures $(\sigma=\mathrm{O}(10))$. Therefore, neglecting the nonlinearity in $(\vec{u} \cdot \nabla) \vec{u}$ in Eq. 2.6 is an acceptable assumption in the case of fluids.

\subsection{Physical Characterisation of Ternary Microemulsions}

The term microemulsion has been introduced by Hoar \& Schulman 47] who firstly examined the comprehensive phase complexity of mixtures comprising water, oil, and surface-active-agents as a function of the constituents' concentration and thermodynamic parameters. Surface-active-agents, commonly abbreviated as surfactants, are amphiphiles, i.e. molecules which possess a hydrophilic head group and a lipophilic tail. These molecules occupy the interface between the oil and the water phase leading to meso-scale structures.

A representative of such meso-structured fluids are microemulsions which are thermodynamically stable and optically clear solutions of surfactant stabilised water (oil) droplets in an oil (water) continuum. This state is referred to as a microemulsion since the size of the minor phase droplets $(\mathrm{O}(10 \AA))$ is orders of magnitude smaller than in regular emulsions $(\mathrm{O}(1 \mu \mathrm{m}))$. 
If not stated otherwise, it will be referred to the ternary water-in-oil $(w / o)$ systems consisting of deuterium oxide, decane, and the surfactant sodium bis(2-ethylhexyl)sulphosuccinate known as AOT (water, decane, AOT abbreviated as WDA). This system is particularly appealing to experimental investigations due to its structural simplicity and a comparatively extensive documentation in literature.

\subsubsection{Micro-Structure}

Amphiphilic molecules dissolved in an oil-continuum spontaneously self-associate, forming spherical structures. Adding water to such dispersions results in "swollen" inverted micelle 1 , where a water droplet is enclosed by a monolayer of surfactant (see Fig. 2.4). As a result of the amphiphile reducing the interfacial tension and a balancing effect by the increased entropy of dispersion, these systems are thermodynamically stable contrary to regular emulsions 85 , 18.

The water core radius of a micell $a$ (see Fig. 2.4) incorporating the surfactant head group region (which is inter-penetrated by water molecules 66] ) is essentially defined by the ratio of the number densities of $\mathrm{D}_{2} \mathrm{O}$ and AOT molecules which is given by the molar ratio of water to surfactant

$$
\omega=\left[m_{A O T} / m_{D_{2} O}\right]
$$

Simple geometric arguments based on the specific volume of a $\mathrm{D}_{2} \mathrm{O}$ molecule $v_{D_{2} O}$, the volume of the water penetrated portion of a single AOT head group $V_{H}$, and the surface area per AOT head group $a_{0}$ lead to a linear relation of the mean radius of the micelles and the molar ratio 68 .

$$
\bar{a}=\frac{3 v_{D_{2} O}}{a_{0}} \omega+\frac{3 V_{H}}{a_{0}}
$$

The surfactant shell thickness meets the average surfactant tail length yielding micellar radii of $\mathrm{O}(10 \AA)$ depending on the composition of the microemulsion.

The second composition related parameter defining the global state of the microemulsion is the volume fraction

$$
\eta=\frac{V_{D_{2} O}+m_{A O T} \cdot \rho_{A O T}^{-1}}{V_{D_{2} O}+m_{A O T} \cdot \rho_{A O T}^{-1}+V_{C_{10} H_{22}}}
$$

which denotes the micellar concentration. As a peculiarity of WDA compared to other microemulsion systems, the individual micellar character is preserved for volume fractions up to $\eta=0.5$ below $T=25^{\circ} \mathrm{C}[22$. However, depending on $\eta$ and $T$ the intrinsic length scale of the system is larger than a single micellar

\footnotetext{
${ }^{1}$ For historical reason spherical collocations of surfactant molecules with the lipophilic tails pointing at the centre are referred to as micelles and the reversed configuration as inverted micelles.
} 


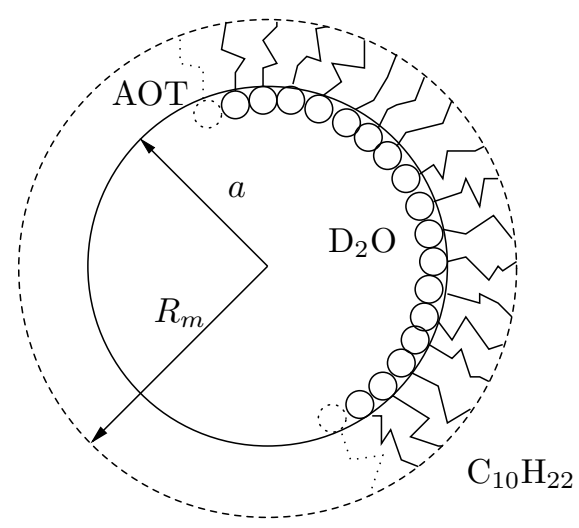

Figure 2.4: Sketch of the $w / o$ micellar micro-structure of the WDA microemulsion.

diameter as the mean aggregation of several micelles leads to the formation of clusters associated with a hydrodynamic radius $R_{h}>R_{m}$.

Individual micelles adhere to adjacent micelles due to molecular bounds of the lipophilic tails of the surfactant shell. Small-angle neutron scattering measurements suggest a strong short-range interaction caused by surfactant tail inter-penetration unlike the rather long-ranging interactions in classical (van der Waals) fluids [51, 66. However, the solvent exhibits an additional cooperative effect by means of a temperature dependent shielding of the micellar surfaces. This shielding allows for the linear $C_{10} H_{22}$ molecules to arrange themselves with respect to surfactant tails $[52$ which generally results in temperature dependent micellar interaction. Furthermore, the interaction is found to depend on the hydrodynamic radius which in turn defines the inter-micellar contact area 17.

In summary, the WDA microemulsion might be intuitively thought of as a suspension of nano-size particles or macro-molecules associated with a thermodynamic interaction potential [15, 52]. This picture is directly analogous to suspensions of nano-size colloids and both systems can be regarded as binary mixtures of particles or micelles and the solvent. This simplified representation of WDA has been found to hold for volume fractions below $\eta \geq 0.2$ while beyond that threshold the micellar aggregates form higher order structures or lose their individual character to bicontinuous phases [21.

\subsubsection{Thermodynamics of Microemulsions}

The WDA system is well-known to exhibit a thermodynamic critical point in terms of a consolute point close to room temperature (cp. [51]). Figure 2.5] sketches the phase diagram of the WDA microemulsion in the plane of temperature versus volume fraction. Below the coexistence curve the microemulsion 


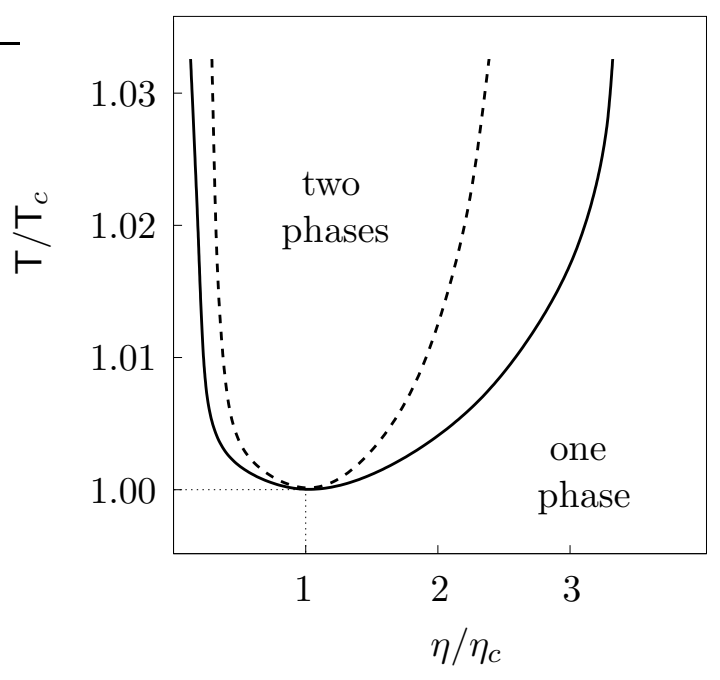

Figure 2.5: Sketch of the coexistence curve (solid line) of the WDA system and the spinodal (dashed).

consists of a single homogeneous phase whereas at higher temperatures two microemulsion phases with different volume fractions coexist. The coexistence curve itself (the binodal) borders the stable mono-phase state against a meta-stable region where sufficiently large concentration fluctuations, i.e. fluctuations exceeding the nucleation threshold, can grow. The spinodal (the bold dashed line in Fig. 2.5) limiting the meta-stable state, denotes the border of absolute instability. Beyond the spinodal, infinitesimal concentration fluctuations infinitely grow and the phase separation occurs by means of spinodal decomposition. At the consolute point, defined by the critical volume fraction $\eta_{c}$ and critical temperature $T_{c}$ both phases become identical.

Generally, the consolute point of a binary mixture is a second-order phase transition determined by the vanishing of the first two derivatives of the chemical potential difference, $\mu=\mu^{(+)}-\mu^{(-)}$with respect to the concentration $c$

$$
\partial_{c} \mu=\partial_{c}^{2} \mu=0
$$

where the superscripts $(+)$ and $(-)$ denote the different phases. Equation 2.23 implies an infinite increase of the susceptibility, $\chi=\partial_{\mu} c$ (the osmotic compressibility) which leads to a very anomalous behaviour of the fluid in approaching $T_{c}$ 39, 3 2.

The critical region, the immediate vicinity of $T_{c}$, is characterised by increasingly strong local concentration fluctuations. More rigorously, the osmotic compressibility is related to the integral of the particle-pair correlation func-

\footnotetext{
${ }^{2}$ The osmotic compressibility remains finite in real systems due to gravitation limiting the growth of concentration fluctuations
} 


\subsection{Physical Characterisation of Ternary Microemulsions}

tion by the fluctuation theorem 35]

$$
\int G_{p p}(\vec{r}) d \vec{r} \sim \chi
$$

which implies the size of the concentration fluctuations to diverge according to the divergence of $\chi$ in approaching $T_{c}$. The size of the fluctuations is given by the half-width of $G_{p p}(\vec{r})$ which is the correlation length $\xi$. Defining the distance to the critical temperature as $\epsilon=\left(T_{c}-T\right) / T_{c}$, the correlation length diverges according to a simple power law $\xi=\xi_{0} \epsilon^{-\nu}$, with $\nu=0.63$ for WDA [51].

It should be noted that the growth of the correlation length $\xi$ can be directly observed in terms of a phenomenon known as critical opalescence. An initially clear solution becomes strongly reflective in the vicinity of $T_{c}$. The clouding of the fluid indicates that the correlation length $\xi$ grows beyond length-scales comparable to the wave length of visible light.

The description of the consolute point in a molecular binary mixture can be directly translated to microemulsions. Neutron scattering measurements of the WDA microemulsion have demonstrated the micellar structures to be conserved throughout the critical region [118, [52. Thus, the phase transition is caused by micellar aggregation only while the individual micellar character is preserved and the volume fraction is equivalent to concentration. The volume fraction is the order parameter of the system. Furthermore, the shape of the binodal can be associated with the micellar interactions. The asymmetry of the binodal is for example a common feature of temperature dependent interaction potentials [2]. Phase separation at higher temperatures and a mono-phase state at low temperatures is associated with strongly oriented intermolecular bonds of the solvent and the micelles. The bonds break off for increasing temperature and entropy, causing phase separation at a lower consolute point [59.

However, along with the divergence of $\xi$ and $\chi$ the thermodynamic and kinetic parameters of the system such as the isobaric specific heat $C_{p}$, the diffusivity $D$, etc. exhibit power law behaviour in the critical region superimposing background terms, terms linear in $\epsilon$, and dominating the thermodynamic quantity $X$ according $X \sim X_{0} \cdot|\epsilon|^{c}$, where $X_{0}$ is the critical amplitude and the exponent $c$ is referred to as a critical index.

Renormalisation group theory states that the critical indices are universal for systems of equivalent space dimensionality and number of parameters, implying liquid-vapour phase transitions in simple fluids and consolute critical points in binary mixtures to belong to the same universality class as the ISING model of ferro-magnetism [59]. Accordingly, the lower consolute point of the WDA system has been demonstrated to be "Ising-like" by the experimental determination of critical exponents. Particularly, the correlation length divergence has been studied by means of neutron scattering experiments [51, 60, 66, 67, 68] along with measurements of the susceptibility and the coexistence curve [118]. 
The static Ising model of ferro-magnetism is based on the simplified conception of a cubic lattice of cells, each carrying a spin, in an external magnetic field, considering nearest neighbour interactions only. Several further extensions like next-nearest neighbour interaction and spreading over into neighbour cells are conceivable and might be accounted for as well. The order parameter in this particular case is the magnetisation 3 . The three-dimensional Ising model cannot be solved analytically but the system thermodynamics close to the critical point can be calculated by means of the renormalisation group theory. As a consequence of the equivalence to the Ising model, the critical indices for the thermodynamic and kinetic parameters of the WDA system are known and the critical behaviour of the transport properties relevant to Rayleigh-Bénard convection can be estimated in analogy to molecular binary mixtures.

Finally, it should be noted that the measured critical indices are depending on the thermodynamic path approaching the critical point on. In the remainder the critical isochore is considered which is most convenient for experiments. According to FisHER [36], varying the temperature at fixed concentration, requires renormalisation of the critical indices. However, the renormalisation has no qualitative impact on the parameter divergence in the critical region and therefore, it will not affect the argumentation concerning the transport properties.

\subsubsection{Thermodynamic Model Systems}

Qualitatively, the coexistence curve similar to Fig. 2.5 can be deduced from a semi-heuristic formulation of the free energy, incorporating contributions by the bending energy of the surfactant mono-layer, the entropy of mixing and the interaction potential [90. Beside from the phenomenological approach, the system's thermodynamics can be deduced directly from the microscopic structure. This approach shall be emphasised because, along with the static micro-structure, it is the constitutive reason to draw an analogy to colloid solutions, which will be useful in estimating the hydrodynamics of microemulsions. Two different models have been employed successfully for the description of WDA which share the idea to regard the micelles as identical spherical hardcore particles with a temperature dependent interaction potential.

The asymmetric coexistence curve can be reproduced by the adhesive hardsphere model (AHS, sometimes also referred to as Baxter's sticky spheres) [10]. The AHS model is based on an attractive square-well pair-potential in the limit of vanishing extent of the well. The model is characterised by a sin-

\footnotetext{
${ }^{3}$ In close analogy to this, LEE \& YANG [76] introduced the term "lattice gas" considering atoms and "holes" (empty spaces) on a regular spatial lattice in order to study gas-liquid systems. To this end, the spin configuration, the magnetisation and the external field are identified with atom-hole pairs, particle density and pressure retaining the spatial lattice structure.
} 


\subsection{Rayleigh-Bénard convection in Microemulsions}

gle parameter which is referred to as the "stickiness". The structure factor (the Fourier transformation of the correlation function) and the coexistence curve can be calculated in the Percus-Yevick approximation, which relates the direct correlation function and the interaction potential [84]. The model predicts a phase transition at a low but slightly overestimated volume fraction of $\eta_{c} \simeq 0.12$ as compared to the literature value for $\mathrm{WDA}, \eta_{c}=0.073$. However, the phenomenology of the phase transition in WDA can be correctly reproduced. It is understood that in the critical region micelles form clusters of growing extend [69] which is in agreement with numerical molecular dynamics simulations [111, 52.

The assumption of an infinitesimal thin attractive well is rather unphysical. Therefore, more recently, BOUASKARNE et al. [15, have proposed a hard-core Yukawa fluid model with an interaction potential of the form

$$
\phi(r)= \begin{cases}\infty & r \leq a \\ -b \frac{e^{-z(r-a) / a}}{r / a} & r>a\end{cases}
$$

wherein $a$ is the hard core diameter and $b$ and $z$ are parameters of the strength and range of the interaction potential. Thereof, the thermodynamics of the microemulsion can be derived based on the inverse temperature expansion of the free energy in the mean spherical approximation. The model correctly reproduces the experimental coexistence curve of WDA and gives an improved prediction of the critical volume fraction $\eta_{c} \simeq 0.8$ which is very close to the experimental values [22].

The micellar clustering in the critical region is consistent with the conception of transient networks being formed which give rise to a large enhancement of conductivity leading to the phenomena of percolation [34]. Furthermore, it should be noted that the clustering is accompanied by a growing polydispersity of the micellar aggregates.

\subsection{Rayleigh-Bénard convection in Microemulsions}

\subsubsection{Rayleigh-Bénard convection in Microemulsions}

In a series of articles LóPEz-QUINTELA, KoRnETA, and others have reported on the investigation of Rayleigh-Bénard convection in different ternary and quaternary $w / o$-microemulsion systems [80, 81, 64, 65].

These authors have studied the convective pattern formation in a cylindrical cell of large aspect ratio using shadowgraph visualisation. The experimental procedure for their investigations was to increase the bottom plate temperature $T_{b}$ over a comparatively large interval of $20 \mathrm{~K}$ in approaching the phase transition temperature $T_{b} \rightarrow T_{c}$ while keeping the top plate temperature constant. Therefore, the pattern morphology has not been studied primarily with regard to the distance of the mean temperature to the critical temperature $T_{c}$. The observed pattern belong to the Rayleigh regime at rather large Rayleigh 
numbers, i.e. large thermal forcing, which imposes continuous mixing and suppresses concentration effects. Furthermore, the thermodynamic scaling $\Delta T / T_{c} \ll \epsilon$ is violated for the experiments due to the large temperature difference $\Delta T$. Therefore, non-Boussinesq effects and the effects of the critical behaviour cannot not be separated.

For the case of a water-cyclohexane-diethylenglycolmonobotylether microemulsion with a comparatively large volume fraction of $\eta \simeq 0.36$ and a molar ratio of $\omega \simeq 10$, five distinct patterns have been differentiated.

Initially, a state of localised travelling waves at the lateral boundaries has been found followed by homogeneous travelling wave (TW) convection in the whole cell. Further increasing of the bottom temperature has lead to the formation of a stationary roll state at the lateral boundaries coexisting with the TW state in the cell centre before superseding the TW state. The fifth pattern, forming for $T_{b}$ being very close to the critical temperature $T_{c}$ has been reported to exhibit polygon shaped structures which are interpreted as a superposition of differently oriented horizontal roll modes.

The first to the fourth pattern correspond to the convective structure evolution of a binary mixture with negative Soret coupling $(\psi<0)$. In agreement with molecular binary mixtures with $\psi<0$, the onset of the TW state is found to be hysteretic. However, the observation of $\mathrm{TW}$ is quite counter-intuitive because, empirically, macro-molecules such as micelles are known to migrate towards colder regions, suggesting a positive Soret effect analogous to the case of classical hard-sphere mixtures [70].

Although a microscopic model for the Soret effect is not available at the time, thermal diffusion has to be associated with both the micell-micell interactions and cross-interactions between the solvent and the micelles. One possible microscopic source of the Soret effect is an interfacial tension driven mechanism, proposed for colloidal dispersions [106] which has been successfully employed for the description of charged micellar solutions [98. In the case of hard-sphere mixtures without electrostatic forces it is generally understood that the heavier component, or for comparable species masses the larger component, diffuses towards the colder regions as long as weak interactions are negligible [117]. With a view to the WDA system, the Soret effect can be expected to be dominated by the short-ranging attractive interaction potential pointing at $\psi>0$ in agreement with surfacted colloid dispersions which also exhibit normal Soret coupling [77]. However, the microemulsion utilised for the convection experiments of KORNETA and others cannot be regarded as a dilute solution and the Soret coupling is known to strongly depend on the type of surfactant and especially the volume fraction. Employing the sticky hard-sphere analogy, the separation ratio is positive for dilute suspensions but a change of the sign of $\psi$ has been predicted for volume fractions exceeding $\eta \simeq 0.1$ [32].

The fifth polygon-shaped pattern is attributed to critical effects. This explanation is sustained by the very strong shadowgraph signal reported, allowing 


\subsection{Rayleigh-Bénard convection in Microemulsions}

for the direct observation of the structure. The strength of the refractive index signal in this regime, i.e. the visibility, has to be attributed to large concentration difference entering the interface between adjacent rolls. The transition from rolls to polygon structures has been reported to be hysteretic, also indicating the latter state to result from incipient phase separation which is suppressed by the shear forces induced by roll structures. Further on, KoRNETA et al. [64, 65] have observed higher concentration droplets modulating the polygon structures. These have been interpreted as "water-rich" dense plumes chaotically rising from the concentration boundary layer at the bottom. Theses dense structures fail to follow the mean flow, thereby causing irregular disruptions of the basic state. The droplets indicate phase separation and thereof it follows that the Boussinesq approximation is not valid for this state of two-phase flow.

It is important to note that the morphology of the polygon-shaped structures can be reproduced numerically on the basis of a Ginzburg-Landau type equation of the form of Eq. 2.15 13. In order to incorporate the effects of criticality into the amplitude equation an additional forcing term is added to reflect the increasing concentration fluctuations and the resulting fluid parameter variations. However, this particular approach is rather heuristic and a microscopic picture based on the impact of concentration effects for example cannot be drawn from it.

\subsubsection{The Colloidal Suspension Analogy}

A more general feature of macro-molecular or micellar suspensions is the very small particle mobility (expressed as the Lewis number $L$ ) as compared to molecular binary mixtures. Accordingly, the vertical solutal relaxation time $\tau_{v, D}=d^{2} / D$ increases and the concentration gradient can be expected not to develop into a linear form according to the equation of state for the Boussinesq approximation on realisable experimental observation times. The vertical concentration profile is characterised by a slowly developing concentration boundary layer at the lower boundary [107]. This problem has been addressed with a view to colloid dispersions exhibiting relatively large separation ratios $(\psi=\mathrm{O}(1))$. A particularly interesting observation is that the onset of Soret driven convection is observed in considerably shorter time than the diffusion relaxation suggests, i.e. in absence of a fully developed concentration gradient [19].

RYSKIN et al. 107 have considered the case of a Lewis number of $L=\mathrm{O}\left(10^{-4}\right)$ and $\psi=\mathrm{O}(1)$ in the framework of a linear stability analysis of the Boussinesq approximation. These authors have derived an expression for the linear growth rate of convective fluctuations $\lambda_{c o}$ as a function of $\psi$ and $L$ based on a low-mode Galerkin approximation. The growth rate is found to be positive below the critical Rayleigh number for the onset of pure fluid convection $(r=1)$ in absence of a relaxed concentration profile. It is argued 
that for large enough Rayleigh numbers $r_{c}<r \leq 1$ the growth rate $\lambda_{c o}$ will depend on $L \psi$ instead of $L$ alone. Graphically, the large buoyancy forces of concentration fluctuations at large separation ratios can amplify convective fluctuations. Therefore, the formation of the vertical concentration profile in response to the temperature gradient can be bypassed leading to stationary Soret driven convection sustained by the convective concentration profile. Once initiated the growth of the concentration profile is proportional to $e^{\lambda_{c o} t}$, as for the temperature and velocity amplitude. The convection amplitude will grow to saturation in a nonlinear stationary state.

The condition for the separation of time scales into purely conductive and fluctuation induced convective ones is, that convective perturbations grow much faster than diffusion, i.e. $\left|\lambda_{c o}(r)\right| \gg L$. In other words, the critical Rayleigh number $r_{c}$ is experimentally not necessarily accessible due to excessive relaxation times. Instead, the onset of measurable convective motion indicates the system to switch its time scales at $r_{c} \leq r<1$. The same argumentation also holds for microemulsions provided the separation ratio is large enough.

The bifurcation behaviour of colloid solutions with a positive Soret coupling has been investigated by HukE et al. [53] based on the Boussinesq approximation. As a main result the bifurcation scenario is qualitatively equal to the case of molecular binary mixtures. Except for the fact that the stability of stationary rolls is shifted to larger Rayleigh numbers $(r>1)$ when the Lewis numbers is small or the separation ratio is large. The region of stable cross-rolls is enlarged accordingly. The convection amplitude in the transition regime from the Soret to the Rayleigh mode approaches the pure fluid solution for decreasing $L$, while raising $\psi$ has the opposite effect. Finally, Soret convection sets in at $0 \simeq r \ll 1$ for values of $L$ and $\psi$ relevant to colloidal solutions provided the vertical concentration profile is relaxed. In the case of $\psi<0$ neither TW nor stationary convection is possible due to the large stabilising effect of $\psi=\mathrm{O}(1)$ [53].

\subsubsection{Convection in the Vicinity of the Consolute Point}

The critical divergence of the correlation length $\xi$ and the simultaneous slowing down of the systems in the vicinity of the critical point causes the transport properties of the fluid to diverge when $T_{c}$ is approached. In order to estimate the parameter behaviour in the critical phase transition region only the diverging terms will be considered and contributions from background terms, linear in $\epsilon=\left(T_{c}-T\right) / T$, will be neglected.

The divergence of the mass diffusion can be related to the correlation length 


\subsection{Rayleigh-Bénard convection in Microemulsions}

divergence by the Stokes-Einstein relationt

$$
D=\frac{k_{B} T}{6 \pi \mu \xi} \sim \epsilon^{\nu}
$$

where $\nu=0.63$ is the critical index of the correlation length and $\mu$ is the dynamic viscosity which, according to dynamic renormalisation-group theory, is related to $\xi$ as 109 .

$$
\mu \sim \xi^{z} \sim \epsilon^{-\nu z}
$$

The universal critical index $z$ is approximately 0.054 , leading to a very weak divergence of $\mu$ with the exponent $(\nu \cdot z)=0.03$ which has been verified experimentally for WDA [11].

A similar argument as for $D$ would hold for the thermal diffusivity $\kappa$ in pure fluids close to liquid-vapour critical point. There, according to dynamic scaling theory, the thermal conductivity takes the form

$$
\lambda=\frac{k_{B} T \rho}{6 \pi \mu \xi} C_{p}+\lambda_{b}
$$

where $\lambda_{b}$ denotes contributions of the background. Both quantities $\kappa$ and $\lambda$, are strongly diverging in simple fluids as $\xi \sim \epsilon^{-\nu}$ and $C_{p} \sim \epsilon^{-\nu}$. However, in binary mixtures close to a consolute point the situation is more complicated due to the coupling of concentration and heat fluxes. The development of $C_{p}$ at a liquid-liquid critical point of a binary mixture is isomorph to $C_{v} \sim$ $\epsilon^{-\alpha}$ for liquid-vapour phase transitions in pure fluids. Due to $\alpha=0.11, C_{p}$ diverges more weakly than the correlation length $\xi$, causing the first term in Eq. 2.28 to vanish for $T \rightarrow T_{c}$. Additionally, mode-coupling theory shows the background, the terms linear in $\epsilon$, to contribute to the critical behaviour of the thermal conductivity. Thus $\lambda$ is enhanced close to $T_{c}$ but remains finite [3, 4] in agreement with experimental results on binary mixtures [38. There is no experimental data available for the heat transport parameters of the critical WDA microemulsion. However, PEPIN et al. did not detect a singular contribution to $C_{p}$ in their experiment on WDA suggesting a very weak amplitude of the divergence [97.

The thermal diffusion $D_{T}$ is predicted not to diverge [117]. Hence, the thermo-diffusion ratio obeys the scaling form

$$
k_{T}=\frac{D_{T}}{D} \sim \epsilon^{-\nu}
$$

neglecting weak contributions. At the same time $k_{T}$ defines the critical behaviour of the separation ratio $\psi \sim \epsilon^{-\nu}$ because the thermal and solutal expansions are identically divergent [42. Finally, the density $\rho$, which is expected

\footnotetext{
${ }^{4}$ The applicability of the Stokes-Einstein relation can be derived more strictly from modecoupling theory, but it is intuitively convincing and it might be referred to 48 for a more rigorous treatment.
} 
to obey the scaling form $\rho \sim \epsilon^{1-\alpha}$, has been found to be effectively linear in $\epsilon$ for ternary microemulsions [4].

In summary, the scaling of the onset of the Rayleigh mode at $R_{c}^{p}$ can be estimated to take the form $R_{c}^{p} \sim \epsilon^{2 \alpha}$, whereas the critical Rayleigh number $R_{c} \sim \epsilon^{-2 \nu}$ diverges strongly. As a result, the onset of the Rayleigh mode will be altered in a very narrow range close to $T_{c}$ only or might even not be resolvable. On the other hand, the onset of the Soret mode is predicted to be considerable shifted to lower Rayleigh numbers. Taking into account the considerations of the colloid suspension analogy, this cannot be expected to be observable in a microemulsion. Due to the small Lewis number, the critical Rayleigh number $R_{c}$ is estimated to be practically zero in the off-critical region already. More importantly, the Soret coupling is largely amplified due to the divergence of $\psi$ in the critical region. The diverging diffusion coefficient causes the Lewis number to decrease according to $L \sim \epsilon^{\nu+\alpha}$. Thus, an increasingly emphasised Soret regime can be expected together with a stretched transition from the Soret to Rayleigh mode due to the large contribution to the convective amplitude by the separation ratio $\psi$.

Assenheimer [8] has reported on a very large hysteresis for the primary bifurcation in an isobutyric acid-water binary mixture $(\psi>0)$ close to the consolute point. Square or cross-roll structures were not observed which can be attributed to the diverging concentration relaxation time proportional to $L^{-1} \sim \epsilon^{-(\nu+\alpha)}$. As a result of the apparent chemical instability of the mixture, the variation of $R_{c}^{p}$ was not found to exhibit power-law behaviour either.

Finally, the expected parameter divergences in the critical region is altered by hydrodynamic effects and the presence of a non-negligible temperature gradient over the fluid layer. It is known that static temperature gradients (which in the case of convection are fundamental to the experiment) induce a smearing of the critical region 3. Furthermore, the thermal relaxation of the fluctuations diverges as

$$
\tau_{\xi}=\frac{\mu \xi^{3}}{k_{B} T} \sim \epsilon^{-3 \nu}
$$

leading to the critical slowing down of the overall system from which it is clear that relaxation will not be achieved on experimental time-scales close to the critical point. Finally, impurities in the measurement volume can be expected to either shift the critical temperature as in the case of equilibrium impurities or to smear out the phase transition region if a composition disturbance is localised (i.e. impurities, which do not equilibrate by diffusion due to critical slowing down).

\subsubsection{The Validity of the Boussinesq Approximation}

Assessing the validity of the Oberbeck-Boussinesq approximation for pure fluids Busse [16] linearised the compressible equations for an one-component 


\subsection{Rayleigh-Bénard convection in Microemulsions}

fluid and introduced the deviation parameter

$$
Q=\sum_{i=0}^{4} \gamma_{i} P_{i}
$$

wherein $\gamma_{i}$ expresses the deviation of each of the following parameters from the reference state: density, thermal expansion, viscosity, thermal conductivity and specific heat. The parameters $P_{i}$ are linear functions of the inverse Prandtl number $\sigma^{-1}$. The predictions of this formulation have been confirmed experimentally [1. It is commonly accepted that non-Boussinesq effects should have minor influence for $Q \leq R a_{c}^{R}$, although the deviation from the OberbeckBoussinesq approximation at $Q=\mathrm{O}(1)$ is alreday significant [14].

With a view to the region close to the consolute point of a microemulsion several difficulties arise. Most of the transport parameters are not known a priori. Only their (relative) divergence can be used to estimate the leading terms in $Q$. It should be noted that all the parameters not related to the concentration field have been shown to diverge weakly. The strong divergence of the separation ratio ensures the onset of stationary convection practically at $r=0$ and the violation of Boussinesq approximation is primarily expected in presence of convection where concentration effects are restricted to the boundary layers.

Finally, the osmotic compressibility diverges strongly $\left(\chi \sim \epsilon^{\gamma}\right.$ with the Ising value of $\gamma=1.24[3$ ). If osmotic compressibility becomes important or, more generally, the density and transport properties become $z$-dependent, the inversion symmetry of the system will be broken $(-z \rightarrow z,-w \rightarrow w,-\Theta \rightarrow \Theta)$, most prominently detectable in an inverse bifurcation to hexagonal structures. However, osmotic compressibility effects in a binary mixtures have been argued to be negligible based on the linear stability analysis of convection close to the plait point, i.e. the liquid-vapour critical point of a mixture $(\psi>0)[30$. The argumet is that the zero-mass current boundary conditions at the top and bottom is sufficient to mask critical effects and, thereby, to preserve the validity of the Boussinesq approximation at the onset of convection in the closest vicinity of the plait point. 
2 Theoretical Background 


\section{Materials and Methods}

\subsection{Experimental Arrangement}

Within the scope of this work, two different experimental set-ups were used. The convection pattern visualisation experiments were conducted in a circular, large aspect ratio cell. The second set-up has been designed to meet the particular demands of microscopic velocity measurements.

\subsubsection{The Visualisation Set-Up}

The experimental set-up basically consisted of a heat bath with the convection cell immersed in (Fig. 3.1). The water of the bath was circulated and temperature regulated by a commercial thermostat (Haake, D8). Great care was taken to homogenise the bath temperature by means of fluid contact to large metal surfaces, turbulent mixing in the bulk, as well as efficient isolation of the outside surface. The test fluid was confined between a copper bottom and a sapphire top plate, both $10 \mathrm{~mm}$ in thickness, separated by a high-density polyethylene (HDPE) frame $(d=1.8 \mathrm{~mm}$ in thickness, with an aspect ratio of $Æ=l / d \simeq 33$ where $l$ is the radius). The cavity was sealed by an adhesive stable against $n$-decane (UV16X-2, Masterbond) and the convection cell featured an additional aluminium housing which provided nearly adiabatic shielding at the lateral boundaries. The top plate was in thermal contact to the heat bath while the bottom plate was electronically heated from below, thereby, defining the heat flux through the cell.

The temperatures of the top and bottom plate $\left(T_{t}\right.$ and $\left.T_{b}\right)$ were measured utilising high precision thermistors which were calibrated in situ. One sensor was situated within the bottom plate while the second one and the calibration probe (a PT1000 sensor) were immersed in the heat bath close to the convection cell. The thermistors consisted of a thermo-sensitive semiconductor material with a very high nominal electrical resistance (typically $R=3 \cdot 10^{5} \Omega$ at $T=25^{\circ} \mathrm{C}$ ) and a resistance-temperature relation of the form:

$$
\frac{1}{T}=\frac{1}{T_{0}}+\sum_{i=1}^{4} b_{i} \cdot\left(\ln \frac{R}{R_{0}}\right)^{i}
$$

where $R_{0}$ and $T_{0}$ denote the reference state and $b_{i}$ are the coefficients determined by calibration. $R$ was resolved within $R \pm 0.5 \Omega$ using a digital multimeter (Keithley, DMM 2001), yielding a relative temperature measurement uncertainty of $\Delta T \pm 0.5 \mathrm{mK}$ after calibration, while the absolute deviations 


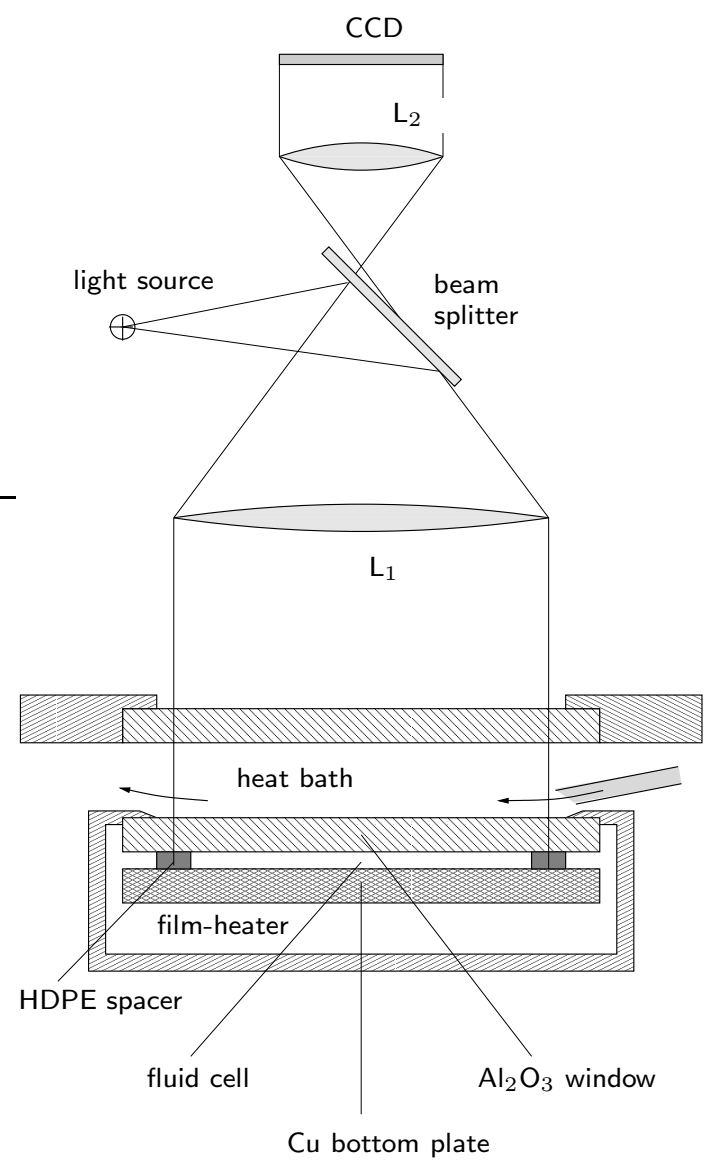

Figure 3.1: Sketch of the Rayleigh-Bénard set-up and the optical arrangement of the shadowgraph system.

emerging from the calibration probe led to $T \pm 20 \mathrm{mK}$. Both the top and bottom plate temperatures were controlled within $2 \mathrm{mK}$ and geometric deviations of the cell spacing were restricted to less than $1 \mu \mathrm{m}$.

The temperature reading and the power supply (Amrel, PPS 1326) for the bottom plate heater were computer controlled via a general purpose interface bus (GPIB) using a MatLab script, in order to gain continuous, automated data acquisition. Therefore, the bottom temperature was subsequently raised in small steps at constant top plate temperature and the estimated thermal relaxation time of the fluid layer was awaited prior to initiating the measurement. Optical access was provided from above in combination with the highly reflective, micro-machined surface of the bottom plate. The optical data acquisition, i.e. shadowgraph image recording, was synchronised with the temperature reading. 


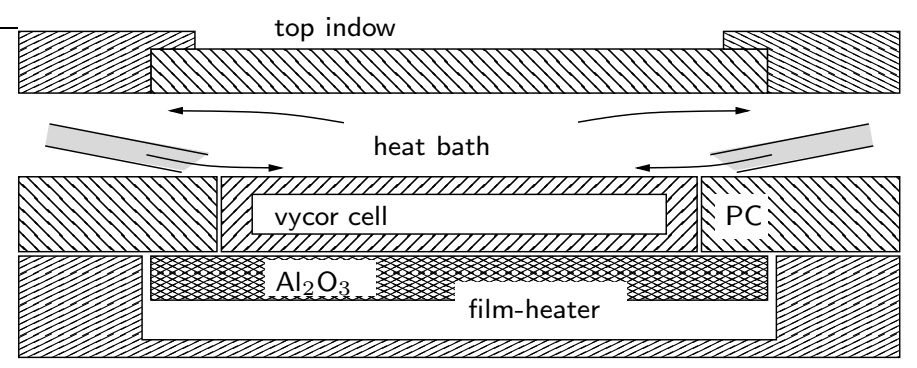

Figure 3.2: Sketch of the Rayleigh-Bénard set-up utilised for direct velocity measurements. The outer enclosure of the heat bath (not shown here) also consists of transparent polycarbonate (PC)

\subsubsection{Set-Up for Velocity Measurements}

The initial idea for the realisation of a Rayleigh-Bénard type experiment suitable for $\mu \mathrm{PIV}$ measurements has been to minimise geometrical deviations, issues of chemical instability, and providing optical access from the sides of the cell by utilising of a fully transparent flow cell immersed into a heat bath.

The core of the Rayleigh-Bénard set-up (Fig. 3.2) was a synthetic quartz cell (Corning Vycor, Hellma) of rectangular base area, with a thickness of $d=3 \mathrm{~mm}$ and an aspect ratio of $1 \times 4 \times 30$. The bottom side of the cell was mirror coated and attached to an electrically heated sapphire bottom plate (Fig. 3.2). From the outside, the convection cell was additionally framed by a $1.5 \mathrm{~cm}$ polycarbonate spacer in order to reduce side wall forcing. The thermal stabilisation of the circulating water was provided by means of a commercial thermostat (Lauda, RE 206) and additional homogenisation in an aluminium cavity filled with steel wool prior to ejection onto the top plate of the cell. The heat bath and the mixing chamber were based on design of DE BRUYN et al. 31 .

The relative top and bottom temperature was resolved within $\Delta T \pm 0.5 \mathrm{mK}$ and the long-term stability was found to be within $5 \mathrm{mK}$ over $12 \mathrm{~h}$. As in the case of the shadowgraph set-up, the temperature measurements were conducted by means of high-precision thermistors in connection with a digital multimeter (Keithley, DMM 2000). The sensors were calibrated in situ, using a PT100 standard probe which caused a slightly larger absolute uncertainty of the temperature reading of $T \pm 0.1 \mathrm{~K}$.

In order to quantify the thermal quality of the flow cell at the vertical boundaries, the heat transfer can be formulated by means of the Biot number which is proportional to ratio of the thermal conductivities of vycor and pure $C_{10} H_{22}$, approximating WDA, $B=\lambda_{\text {vycor }} / \lambda_{C_{10} H_{22}}=\mathrm{O}(10)$. Thus, $B$ was at least one order of magnitude lower than in comparable Rayleigh-Bénard studies. However the value is acceptable to allow for the application of the 


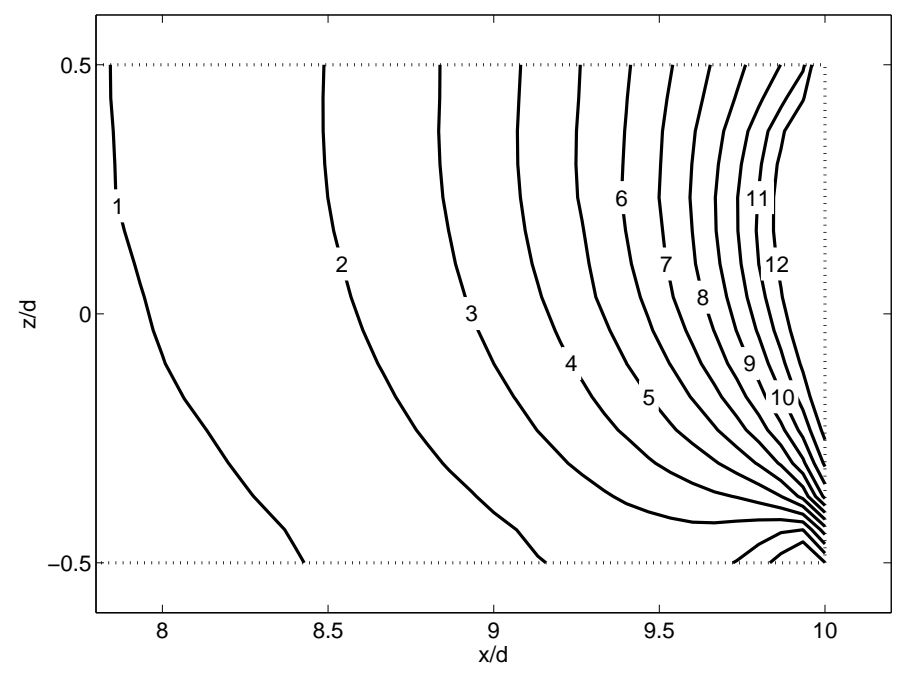

Figure 3.3: Calculated non-dimensional deviation from the linear temperature gradient at the lateral boundaries of the convection cell (in percent). The top and bottom boundaries are at $z / d= \pm 0.5$ and the lateral boundary is at $x / d=10$ (dotted line).

Boussinesq approximation as it will be shown in Sec. 4.1.3.

The side wall forcing might be quantified by the static heat transfer and its deviations from the quiescent conducting state in the cell centre as depicted in Fig. 3.3 In this manner, it becomes apparent that at lateral boundaries, the combination of the flow cell with the outer frame was inferior to adiabatic shielding. The static temperature fields in the absence of convection as well as corrections for the top and bottom side of the cell were evaluated by numerically solving Poisson's law in two dimensions. As the temperatures was probed at the bottom plate and in the heat bath, the measured vertical temperature difference $\Delta T_{m}$ was corrected for losses in the sapphire bottom plate $\left(d_{s}, \lambda_{s}\right)$ and the glass of the convection cell $\left(d_{c}, \lambda_{c}\right)$ according to

$$
\Delta T=\Delta T_{m}\left(1+\frac{2 d_{c}}{d} \frac{\lambda_{C_{10} H_{22}}}{\lambda_{c}}+\frac{d_{s}}{d} \frac{\lambda_{v}}{\lambda_{s}}\right)^{-1}
$$

where the thermal conductivity of decane again serves as an approximation for that of WDA.

Particular measures were taken to avoid contamination of the specimen prior to or during the measurements. Figure 3.4 sketches the arrangement for filling and circulation of the test fluid in the convection cell. Two outer reservoirs $\left(R_{1}, R_{2}\right)$ were used, connected to the fluid cell by teflon (PTFE) tubes, which allowed for fluid exchange without contact to ambient air. 


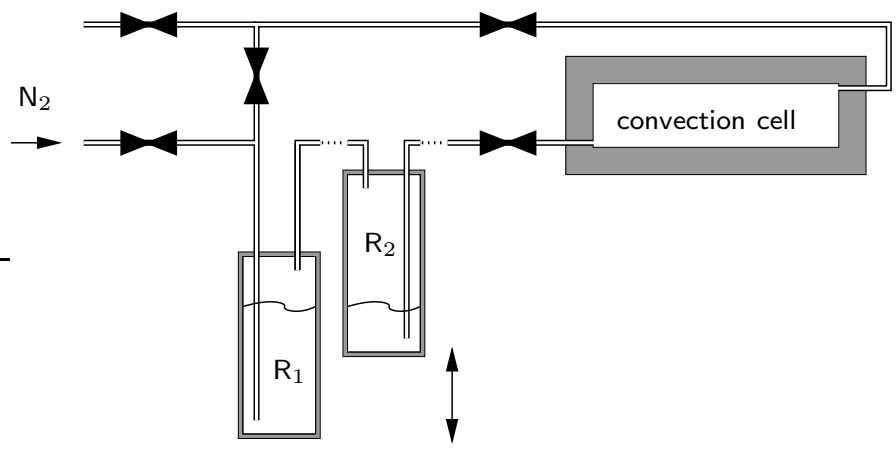

Figure 3.4: Sketch of the experimental arrangement for the handling of highly sensitive fluid mixtures. Pressurised nitrogen $\left(N_{2}\right)$ was used for initial filling of the convection cell in order to avoid contact with atmospheric moisture.

\subsection{Fluid Specimen}

\subsubsection{The Ethanol-Water Binary Mixtures}

The specimen of the binary mixtures were prepared by weight, using ethanol (Riedel-de-Haën, Spectranal, 99.8\%) and water (Fluka, water for organic trace analysis). Alcohol-water mixtures, once prepared, are stable. However, the measurements were conducted immediately after preparation using the transfer systems described above. The thermodynamic parameters of the two different binary mixtures are given in App. 1.2

\subsubsection{The WDA Microemulsion}

The critical microemulsion, consisting of deuterium oxide $\left(D_{2} O\right)$, n-decane $\left(C_{10} H_{22}\right)$, and sodium bis(2-ethylhexyl)sulphosuccinate (AOT), is composed by fixing the molar ratio of water to surfactant to $\omega=40.8$ at a volume fraction of $\eta=0.073$ [105, 151, 118, 83, where the AOT volume is calculated from the density at ambient conditions and volume changes due to dilution are neglected. The preparation of the microemulsion is straight forward due to the spontaneous formation of continuous micellar dispersions of AOT in hydrocarbons. The specimens are prepared by weight, simply adding $\mathrm{D}_{2} \mathrm{O}$ to an appropriate mixture of AOT and $C_{10} H_{22}$. An optically clear solution forms after shaking for approximately one minute 102. All ingredients, AOT (Fluka, Bio-Chemical, Ultra, $\geq 99.0 \%$ ), $n$-decane (Sigma, anhydrous, 99+\%), and $\mathrm{D}_{2} \mathrm{O}$ (Aldrich, "100", 99.96\%) are used without further purification.

The (near-) criticality of the specimen is checked by means of the equal volume criterion. If the specimen is homogeneous and critical the separated phases occupy equal volumes closely above the phase transition tempera- 
ture which can be determined in terms of the meniscus position marking the interface. The critical temperatures are found to scatter in the interval $T_{c} \simeq 30$ to $35^{\circ} \mathrm{C}$. The nature of this variation remains unknown, however, several authors mention specimen of nominally equal composition to exhibit differences in $T_{c}$ as large as a few $\mathrm{K}$ [118, 41, 104]. These deviations are most likely to be associated with impurities or contamination of the reactant, exposition to air and aging. Therefore, the microemulsion specimen were transfered to the convection cell directly after preparation.

The knowledge of the thermodynamic properties of the WDA system is fragmentary, however the known properties of WDA are summarised in App. 1.3.

\subsection{Methods of Investigation}

\subsubsection{Particle Image Velocimetry}

Particle Image Velocimetry (PIV) relies on sequentially imaging particle tracers homogeneously distributed in the flow under consideration. Subsequently, the local velocities are determined by cross-correlation of corresponding subregions of successive images, the so-called interrogation windows, yielding the local tracer ensemble shift. A comprehensive recent review of PIV techniques can be found in RAFFEL et al. 103.

For the PIV measurements the central plane of the convection cell was illuminated from the side using a continuous wave Argon-ion laser (see Fig. 3.2). The flow field at a vertical cross-section of the cell was recorded (at sampling frequencies between $f_{s}=0.5$ and $4 \mathrm{~Hz}$ ) using a long distance microscope (K2, Infinity, Photo-optical Imaging) providing fields of view of approximately $1 \times 1.8 d$ at a magnification of $M=1.2$. The microscope provided sufficiently large working distances to image the flow through the heat bath.

In contrast to conventional $\mu \mathrm{PIV}$, non-fluorescent tracers were used, in order to avoid complications due to tracer-fluid interference. The field of view was optimised with respect to spatial and velocity resolution. Therefore, the lateral extend of the fields typically comprised one roll structure for the wave numbers $k$ relevant to this work. 960 images were recorded for each data point. Issues of measurement accuracy and systematic deviations will be discussed in greater detail in Sec. 4.1.

The tracer particles were either polystyrene micro-spheres (Kisker, PPs-1.0, mean diameter $\overline{d_{p}}=0.992 \mu \mathrm{m}$ ) for the binary mixtures or cross-linked carboxyllatex spheres (Invitrogen, DVB/Carboxyl Latex, mean diameter $\overline{d_{p}}=0.970 \mu \mathrm{m}$ ) in case of WDA. The optimum particle volume fraction can be estimated based on the particle visibility, the illumination and $d_{p}$ with respect to the desired interrogation window size 92 . Hitherto, there is no detailed investigation on the influence of tracers on amphiphile mixtures available. However, the influence of certain tracers on complex mixtures raises questions about fluid-tracer interactions (i.e. surface effects) and the stability of the mixture composition 


\subsection{Methods of Investigation}

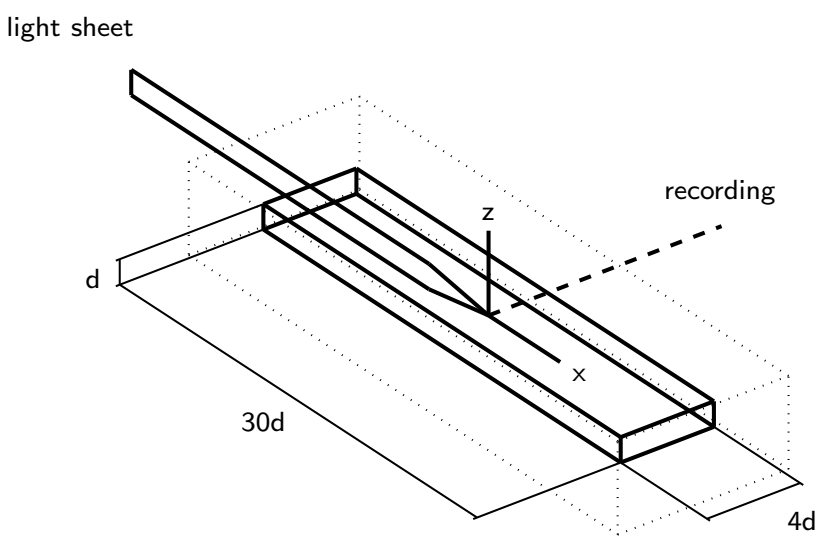

Figure 3.5: Sketch of measurement arrangement for $\mu \mathrm{PIV}$ in the convection cell.

(i.e. criticality). Therefore, the fluid characteristics with and without tracer particles were evaluated by means of dielectric relaxation spectroscopy, reported in Sec. 4.1.2

\section{Velocity Field Evaluation by Proper Orthogonal Decomposition}

The dynamics of a velocity field can be assessed by the application of Proper Orthogonal Decomposition (POD). POD is a common procedure in the identification of coherent structures for example in turbulent flows. The attempt here is different as this work is exclusively concerned with laminar flows on the micro-domain. However, the POD provides the opportunity to inspect stationary velocity fields for time-dependent modulations and, as it will be discussed in greater detail below, it is particularly useful for the evaluation of measurements of instationary flows and flow fields containing non-random localised noise.

The fundamental idea of POD is to expand a time dependent vector field $\{\vec{v}\}$ into a set of orthonormal spatial modes $\left\{p_{i}(\vec{x})\right\} 1$, and time dependent coefficients $\left\{\zeta_{i}(t)\right\}$ which allow for a best possible approximation of the original field.

The optimisation problem of an optimum approximation of $\{\vec{v}\}$, on average in time, corresponds to the eigenvalue problem according to 112

$$
\int d x^{\prime} C\left(\vec{x}, \vec{x}^{\prime}\right) \vec{p}\left(\vec{x}^{\prime}\right)=\lambda \vec{p}(\vec{x}),
$$

\footnotetext{
${ }^{1}$ Strictly speaking, the eigenmodes are the result of a coordinate transformation and have no physical meaning a priori. Their physical relevance has to be argued with respect to the flow field complexity in both space and time.
} 
with eigenvectors $\vec{p}$ wherein $C$

$$
C\left(\vec{x}, \vec{x}^{\prime}\right)=\left\langle\vec{v}(\vec{x}, t) \otimes \vec{v}\left(\vec{x}^{\prime}, t\right)\right\rangle
$$

is the autocorrelation matrix of the vector field $\{\vec{v}\}$. The brackets on the right hand side of Eq. 3.4 denote the temporal average. The expansion is of the form:

$$
\vec{v}(\vec{x}, t)=\sum_{i} \zeta_{i}(t) \cdot \psi_{i}(\vec{x})
$$

The time evolution coefficients $\zeta_{i}$ are calculated by projection of the $\left\{\vec{v}_{i}\right\}$ onto the eigenmodes $\left\{\vec{p}_{i}\right\}$ and the basis modes are uncorrelated.

$$
\left\langle\zeta_{i} \zeta_{j}\right\rangle=\lambda_{i} \delta_{i, j}
$$

Accordingly, a mode represents an aspect of the flow field which is not contained in any other mode. Combining Eq. 3.4, 3.5] and 3.6 yields

$$
C\left(\vec{x}, \vec{x}^{\prime}\right)=\sum_{i} \lambda_{i} p_{i}(\vec{x}) p_{i}\left(\vec{x}^{\prime}\right)
$$

The elements of the basis $\left\{\vec{p}_{i}\right\}$ are denoted as coherent structures. Furthermore, it can be shown that the set of $\left\{\vec{p}_{i}\right\}$ is unique and complete and that the eigenvalues $\lambda_{i}$ represent the projection of the mean energy of the system onto the axis $\vec{p}_{i}$. Accordingly, the mean energy of the entire system $\langle E\rangle$ corresponds to

$$
\langle E\rangle=\sum_{i} \lambda_{i} .
$$

Thus the truncated approximation by the new set $\left\{\vec{p}_{i}\right\}$ can be regarded as an optimised representation of the flow field with respect to the energy proportion of the modes. For a further discussion of the interpretation of the modes and the solvability of Eq. 3.7 it should be referred to [112].

Concerning discontinuous, i.e. discrete PIV vector fields $\left\{\vec{v}_{n}\right\}$ of $N$ images of resolution $M$, separated by discrete time steps $i \cdot \Delta t$, the correlation tensor reduces to

$$
C\left(\vec{x}, \vec{x}^{\prime}\right)=\frac{1}{n} \sum_{i=1}^{n} \vec{v}_{i}(\vec{x}) \vec{v}_{i}\left(\overrightarrow{x^{\prime}}\right)
$$

where the degenerated core has eigenfunctions of the form

$$
\vec{p}=\sum_{i=1}^{n} \zeta_{i} \vec{v}_{i}
$$

Combining Eq. 3.4, 3.9. and Eq. 3.10 yields

$$
C \vec{\zeta}=\lambda \vec{\zeta}
$$




\subsection{Methods of Investigation}

with

$$
\vec{\zeta}=\left(\zeta_{1}, \ldots, \zeta_{n}\right) \quad \text { and } \quad C_{i, j}=\frac{1}{n}\left(\vec{v}_{i}, \vec{v}_{j}\right) \quad .
$$

Note that $C$ is of the order $n$, i.e. the precision of the approximation depends on the number of fields acquired.

It is obvious that such a decomposition aims at the identification of a desirably small number of modes contributing to the field dynamics. With a view to convection, the particularly simple morphology of the flow field results in very few modes of considerable energy content. However, as it will be described in further detail below, the separation of the flow field in independent structures can be regarded as a filter which will be employed for an improved $\mu$ PIV data evaluation scheme (see Sec. 4.1).

\section{Accuracy of the $\mu$ PIV Measurements}

Estimating the systematic errors of the $\mu$ PIV measurements, at first the particles' fidelity in following the flow has to be accounted for. The tracers exhibit a density mismatch of approximately $30 \%$. Assuming Stokes flow conditions, a conservative estimate of the gravitational effects yields a settling motion of the order of $\mathrm{O}\left(10^{-2} \mu \mathrm{m} / \mathrm{s}\right)$. At the same time, the particles are sufficiently large not to be subjected to noticeable displacements due to Brownian motion 92 . A further source of systematic velocity deviations is an inclination (of the angle $\theta$ ) of the convection cell itself leading a to large-scale flow with an vertical profile of the $u$-component of 99

$$
u(z)=\frac{g \alpha \Delta T d^{2}}{12 \nu}(\sin \theta) 2 z^{2}(z-1 / 2), \quad(-1 / 2 \leq z \leq 1 / 2) .
$$

Thus, given $\Delta T \simeq 1 \mathrm{~K}$ an inclination angle of $\Theta \leq 3^{\circ}$ would yield $u(z) \leq \mathrm{O}(0.1 \mu \mathrm{m} / \mathrm{s})$. Therefore, these deviations can be neglected by carefully levelling the cell.

Furthermore, the statistical deviations of the velocity determination can be derived from the optical properties of the measurement system with respect to particle image diameter and correlation resolution, i.e. the interrogation window size. Resolving the particle images by 3 to 4 pixel, the correlation peak can be detected within $1 / 10 d_{p}$ leading to peak location uncertainties of $\delta x \sim d_{p} / 10 M$, where $\bar{d}_{p}$ and $M$ are the mean particle diameter and the magnification of the microscope respectively (92 and references therein). Therefore, the resolution of the velocity signal is found to be limited to $u \pm 0.4 \mu \mathrm{m} / \mathrm{s}$.

However, the local tracer density in the image and out-of-plane motion of the tracers contribute to the background noise, too. In summary theses effects lead to an absolute minimum uncertainty of $\delta u=0.5$ to $0.7 \mu \mathrm{m} / \mathrm{s}$ which can be determined by reference measurements at zero flow $(\Delta T=0)$. Finally, the global error of the velocity and the resulting velocity average, in the case of 
steady flow, is derived from the local probability density function of the fluctuating part $v^{\prime}$ of $v=\langle v\rangle+v^{\prime}$ with $v=\left(u^{2}+w^{2}\right)^{1 / 2}$ and the brackets denoting time average [58].

With a view to ensemble correlation 2 , the deviations are estimated based on the broadening of the cumulative correlation peak width as a function of particle image diameter [58]. The random errors are found to fall below $4 \%$ of the measured value not incorporating effects of tracer density in the image.

\subsubsection{Shadowgraph Visualisation}

Shadowgraph techniques evaluate the deflection of light passing through a field of non-homogeneous index of refraction $n$ where the deflection is proportional to the second spatial derivative of $n$ perpendicular to the line of sight. A comprehensive description of shadowgraph visualisation can be found in SetTles [110.

Figure 3.1 sketches a common shadowgraph typically used in connection with Rayleigh-Bénard convection in thin fluid layers. The cell is illuminated by a high-intensity LED whose light was coupled into the shadowgraph by means of a semi-transparent mirror and parallelised by the collimator lense $L_{1}$. The light is reflected by the surface of the bottom plate, thus, passing through the fluid layer twice. Finally, the light is projected onto the recording CCD by the imaging lense $L_{2}$.

The lateral density variation within the fluid layer of hight $d$ induces a perturbation in the refractive index field of the form $\delta n(x, y, z)$ (which might be thought of as sinusoidal in $x$ and $y$ ). That leads to an intensity distribution at a distance $l$ from the fluid layer:

$$
I(x, y, z=l)=\frac{I_{0}}{1-l / F},
$$

where $I_{0}$ denotes the incipient intensity and $F$ is the focalisation length of the lense-like effects emerging from the local curvature of the refractive field of the fluid pattern given as

$$
F^{-1}=\left(\frac{\partial^{2}}{\partial x^{2}}+\frac{\partial^{2}}{\partial y^{2}}\right) \int_{0}^{d} \frac{\delta n(x, y, z)}{n_{0}} .
$$

Thereof it follows, that the refractive index distortion field might be measured quantitatively if $l$ is kept small enough to allow for the linerasition of the equation for the optical path. Hence, the refractive field, which is related to the temperature field via the fluid density, can be measured quantitatively and the convective amplitude can be determined [89].

However, as density variation in the Soret regime are very weak, especially close to the onset of convection, the shadowgraph is adjusted to maximise the sensitivity. The plane of maximum brightness of the shadowgraph image meets the first caustic at $l \simeq F$.

\footnotetext{
${ }^{2}$ Averaging image pair correlations is commonly referred to as ensemble correlation [103.
} 


\subsection{Methods of Investigation}
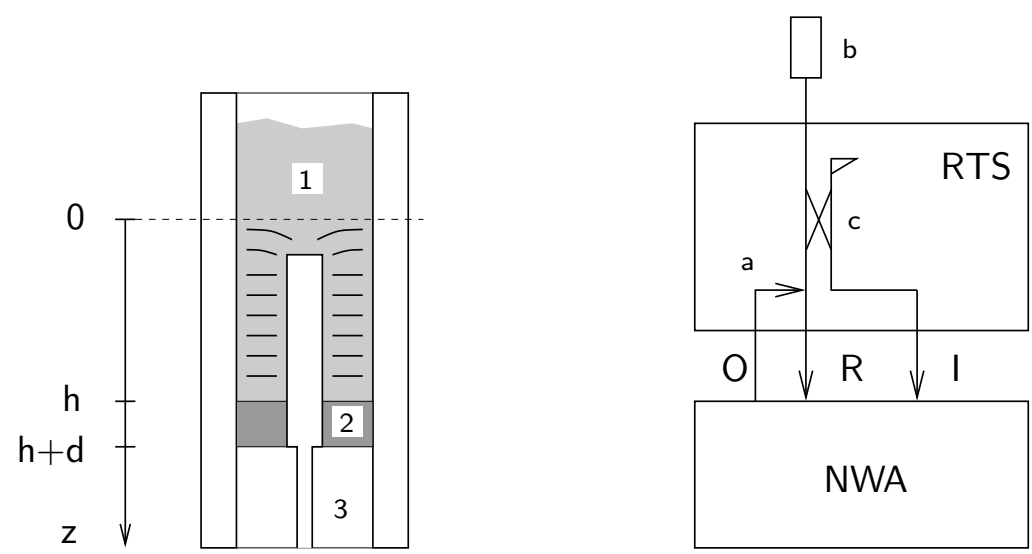

Figure 3.6: Sketch of the coaxial reflexion cell (left) and principal representation of the reflexion factor measurement set-up (right), according to 108 .

\subsubsection{Dielectric Relaxation Spectroscopy}

Dielectric relaxation spectroscopy measures the complex permittivity $\varepsilon$ of a fluid as a function of the frequency of excitation $f$. Physically, it might be thought of the time lag of the dielectric particles (atoms, molecules, macro molecules) present in a fluid in building up an equilibrium polarisation in response to an external electric field. The rotational motion of polar molecules and molecular groups for example leads to orientation polarisation associated with dispersion of the permittivity, i.e., dielectric relaxation. More rigorously, the normalised autocorrelation function $C(t)$ of the macroscopic polarisation $P$ is considered [57]:

$$
C(t)=\langle P(t) P(0)\rangle /\langle P(0) P(0)\rangle \quad,
$$

which is related to the permittivity via a Laplace transformation $\mathscr{L}$

$$
\frac{\varepsilon(f)-1}{\varepsilon(0)-1}=\mathscr{L}\left(\frac{d C(t)}{d t}\right) .
$$

The measurements were conducted by means of reflexion factor determination using an open coaxial cell with its centre conductor abruptly terminated (left hand side of Fig. 3.6). The set-up consisted of a network analyser (NWA, HP 8753), the reflexion test set (RTS, HP 85044A), and the reflexion cell itself (cp. Fig. 3.6). For the measurements, a reference signal is split off the test signal, generated by the NWA $(\mathrm{O})$, at the power divider of the RTS (a). The test signal is reflected in the cell (b), decoupled from the original line by the directional coupler of the RTS (c) and lead to the input of the NWA (I). 
The complex reflexion factor is then determined by comparing the amplitude and phase difference of the test and reference signal. An experimental run comprises measurements at fifty-one frequencies after a calibration procedure based on the reflexion factor measurement for three specific line terminations (short, open, and adapted termination) to compensate for the coaxial lines connecting NWA and RTS. Furthermore, the position of the termination resistance $(R(z=0)=\infty \Omega$, right hand side of Fig. [3.6) which is not known a priori, has to be transformed into the calibration plane $(z=h+d)$ which is the lower surface of the PTFE window of the cell (2). This procedure necessitates two steps, a transformation to $z=h$, the interface of the window and the fluid specimen (1), and a second one to $z=h+d$ introducing two parameters $h$ and $d$ which are determined by a reference measurement in purified water. The permittivity of water is known to be accurately described by a Debye function which is fitted to the data in order to evaluate $h$ and $d$. Thus, an experimental run involved the reflexion cell calibration, the measurement of the reflexion factors of water, cyclohexane, and two specimen of WDA. In between emptying and refilling, the cell was repeatedly rinsed with acetone and dry nitrogen. The reflexion data was evaluated relative to cyclohexane which has a similarly low permittivity as decane. The measurement uncertainties for the spectral range relevant to this work is $\varepsilon^{\prime} \pm 5 \%$ and $\varepsilon^{\prime \prime} \pm 2 \%$ [108]. 


\section{Results and Discussion}

This chapter is subdivided into four parts. Prior to presenting the results for convection in binary and pseudo-binary mixtures the enhancements of the velocity measurement technique will be described. The second part covers a detailed investigation of both stationary and travelling wave convection in a binary mixture where the thermodynamic parameters are known. Subsequently, velocity measurements in three different compositions of WDA will be discussed. The final part will deal with convection close to the consolute point of WDA, studied by means of pattern formation visualisation.

\subsection{The Adaption of the $\mu$ PIV Technique}

The particularities of $\mu \mathrm{PIV}$ utilising non-fluorescent tracer particles on mediumscale length scales $(\mathrm{O}(\mathrm{mm}))$ primarily require an adaption of the data evaluation as compared to standard $\mu \mathrm{PIV}$ methods. The advancements regarding the measurement technique affiliated with this work will be specified below, including a measurement noise reduction scheme based on flow field decomposition and an investigation of the impact of tracer particles on $w / o$ microemulsions. The global quality of the experimental design is inspected by a reference measurement of convection in pure $\mathrm{C}_{10} \mathrm{H}_{22}$.

\subsection{1 $\mu$ PIV Data Evaluation}

The combination of microscopic imaging and non-fluorescent tracers might be regarded as a hybrid of macroscopic and $\mu \mathrm{PIV}$ which is more related to PIV methods using infrared wave lengths than to conventional $\mu$ PIV. Generally, the application of microscopic imaging leads to an increased noise level compared to macroscopic PIV, mainly due to the depth of focus which is smaller than the depth of illumination. Particle image blurring by out-of-focus particles is unavoidable for the required tracer concentrations, decreasing and smearing out the correlation signal.

Background as well as reflective noise at the boundaries of the flow field cannot be filtered selectively. Commonly, the inferior quality of $\mu$ PIV raw data is compensated for by averaging image series either with regard to intensity, velocity or correlation provided that the flow can be presumed to be stationary [92. Preferably, the pairwise correlation can be averaged, i.e. ensemble correlation should be applied, as the statistical noise reduction is simply a function of the series length. However, it turns out that ensemble correlation is not fully sufficient for the evaluation of convective structures incorporating areas of very low speed. Especially, the vertical boundaries of the cell are 


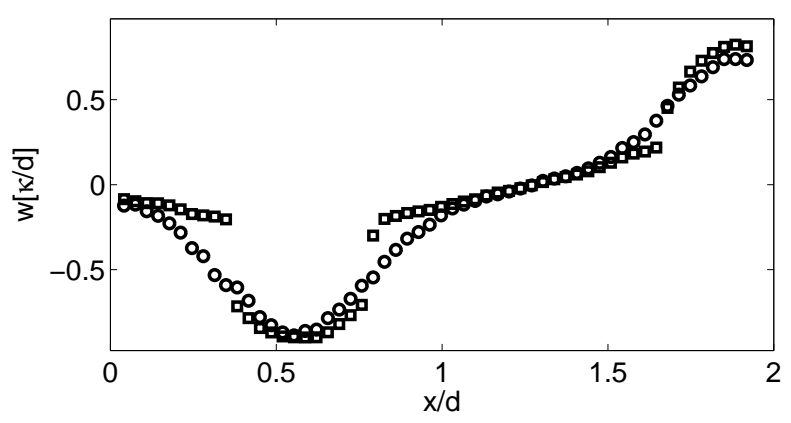

Figure 4.1: An example of the lateral velocity profile at $z / d=0$ : $\square$ denote ensemble correlation evaluation and $\bigcirc$ represent POD filtered evaluation.

found to impose a severe disturbance onto the interrogation in their closer proximity. Surface reflexions and sticking particles lead to erroneous correlation distributions which are obviously non-Gaussian, i.e. they cannot be compensated for by correlation averaging. A similar argument holds for the core region of the convective roll structures. There, the image density is locally reduced and the velocity signal approaches the noise level, leading to virtually prolated cores in the velocity fields. This is of particular importance for the analysis of the lateral profiles because a decoupling of the cores induces an overestimation of the nonlinearity of the profiles (Fig. 4.1). In order to reduce the influence of localised, non-Gaussian noise, an evaluation scheme based on a single POD-mode approximation is introduced as an alternative to ensemble correlation.

The idea of the noise reduction scheme for stationary flows based on POD is to decompose vector fields which are not correlated in time as it is usually required. In this case, the constant component of the field will collapse onto the first mode, provided the input fields are sufficiently homogeneous and dense. Statistical noise does not contribute to the correlations and is distributed over all the calculated modes. On the other hand, localised minor coherent modulations of the basic state are covered by higher-order modes. This point shall be emphasised a little further, since the term minor is crucial. Primarily, the energy content of the modulation has to be small with respect to the basic state, i.e. $\lambda_{b s} \gg \lambda_{m}$, where the subscripts $b s$ and $m$ refer to basic state and modulation. Secondly, the tracer density in the image must allow for the distinct identification of the basic mode over the whole field of view. The latter is not necessarily true for $\mu \mathrm{PIV}$ data and it must be assured by sufficiently dense tracer distributions in the flow. In order to illustrate the effect of low image density, Fig. 4.2 shows a representative interrogation window for the case of an ethanol-water binary mixture and $n$-decane at equal nominal tracer concentrations. It is striking that in hydrocarbons the tracer 


\subsection{The Adaption of the $\mu$ PIV Technique}
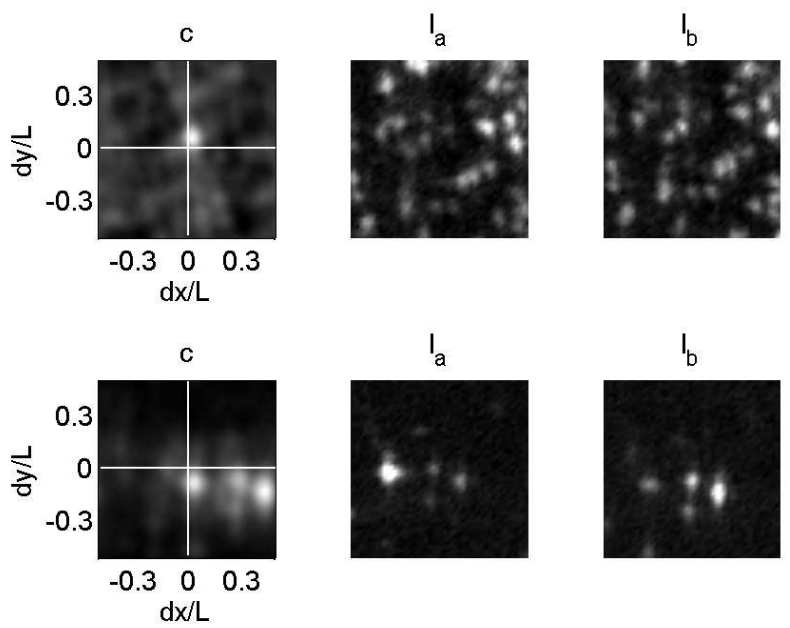

Figure 4.2: Two examples of the image density of a $48 \times 48$ pixel $\mu \mathrm{PIV}$ interrogation window pair $I_{a}$ and $I_{b}$ and the corresponding correlation plane $C$. The upper row is an ethanol-water mixture containing polystyrene spheres; the lower row depicts $n$-decane with crosslinked carboxyl tracers.

density is reduced which is directly associated with an increased aggregation and surface-particle interaction. Furthermore, the lower row of Fig. 4.2 accidentally captures an effect of the very small depth of field which is intrinsic to $\mu \mathrm{PIV}$. The left and right particle of the rhomboid shaped formation seem to exchange intensities due to out-of-plane motion. These virtual velocity fluctuations alter the correlation of the field and impose a strong bias on the average velocity and consequently on the first POD mode. Intensity averaging might partly circumvent this problem.

However, if both conditions are fulfilled (i.e. a sufficient tracer density is provided and the modulation is small with respect to the field) a single mode approximation can be exploited as a velocity field average with an additional filtering of localised fluctuations. The same argumentation holds for a twomode representation of travelling waves.

Finally, it should be noted that for velocity domain operations, a validation criterion for the initial correlations is indispensable. Here, a correlation and a signal-to-noise ratio threshold was defined partly discarding spurious vectors prior to the POD. Additionally, the raw images might be subjected to a highpass filter to remove large-scale background effects.

In order to check the consistency of the evaluation methods with respect to the undisturbed bulk of the fields, the maximum velocities obtained directly from the ensemble averaged data can be compared to the POD-filtered data. The maximum velocities are derived from the supremum profiles of the 


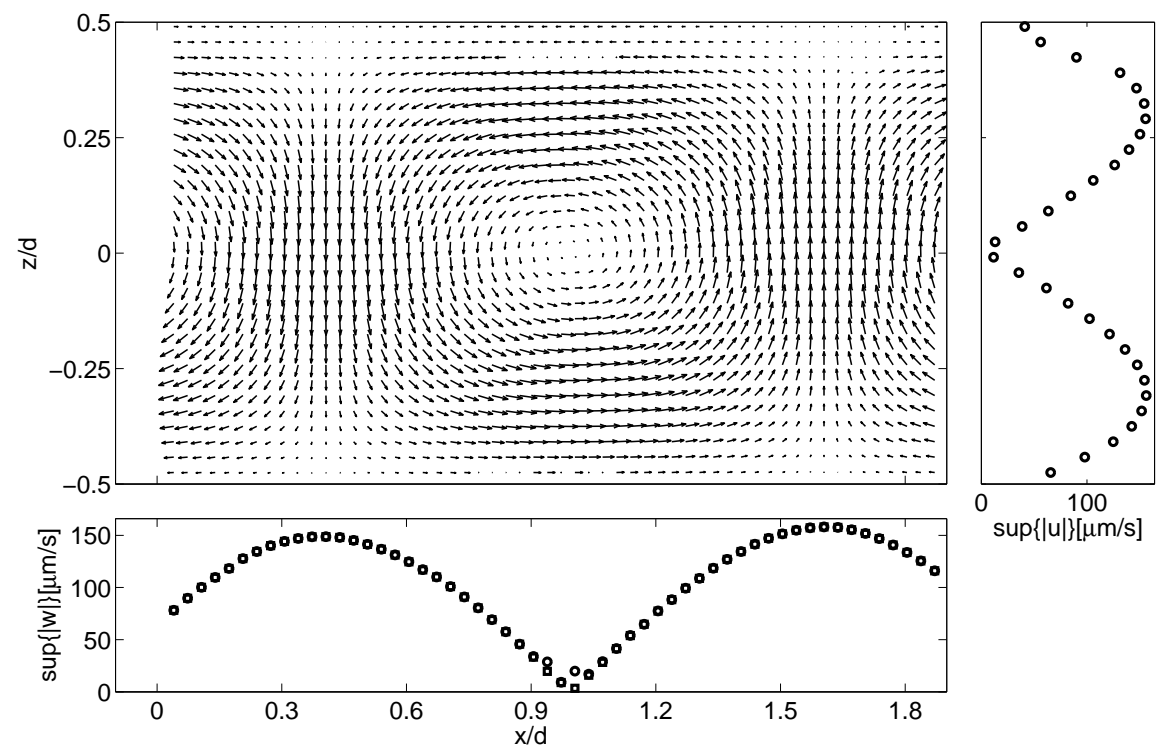

Figure 4.3: An example of the extraction of the maximum velocities in $x$ and $z$-direction. $\sup \{|w|\}$ denotes the supremum of $w$ along the vertical direction at fixed $x ; u$ is treated accordingly. The lower graph presents both $\sup \{|w|\}(\circ)$ and $w(x / d, 0)(\square)$ which nearly perfectly collapse onto the same curve.

absolute values of $u$ and $w$

$$
u(z / d)=\sup \{|u(\forall x / d, z / d)|\}, \quad w(x / d)=\sup \{|w(x / d, \forall z / d)|\}
$$

which are extracted from the fields along the horizontal and vertical direction respectively (see Fig. 4.3). The maximum values of the $u$ and $w$ velocity are identified with the peak amplitudes of the flow. If not stated otherwise, the lateral profiles and the Fourier mode approximation of those is based on POD filtered data.

\subsubsection{The Impact of Tracer Particles}

In order to evaluate the effect of tracer particles on the microemulsion, dielectric relaxation spectroscopy (DRS) is applied to the various specimen of WDA with and without additional tracers. The underlying idea is basically twofold: primarily, DRS allows for the identification of the different relaxation processes, i.e. relaxation times and thereby characteristic meso-structures length scales present in the system. Secondly, the amplitude of the permittivity will be shown to be sensitive to the global water content of the microemulsion specimen. 


\subsection{The Adaption of the $\mu$ PIV Technique}

Adding tracer particles to a microemulsion results in introducing a very large interface with the fluid and, possibly, contamination. Hence, one might expect two major effects to impact on the fluid behaviour. Chemical impurities incorporated in the tracers or entering the systems with them can both affect the micellar concentration (the volume fraction $\eta$ ) and the micellar size proportional to the molar ratio $\omega$. Surfactant molecules might occupy the particles' surface essentially affecting $\omega$. The first primarily changes the amplitude of the dielectric relaxation, whereas the latter alters the micellar size which is proportional to the characteristic relaxation time.

It was chosen to investigate the dielectric relaxation of the critical WDA composition ( $\omega=40.8, \eta=0.073)$ which is expected to be most sensitive to alterations of its constitution. The dielectric relaxation of monodisperse micelles corresponds to the Debye model of re-orientation polarisation

$$
\varepsilon=\varepsilon_{h}+\frac{\Delta \varepsilon}{1-i 2 \pi f \tau_{\varepsilon}}
$$

where $\varepsilon_{h}$ and $\varepsilon_{l}$ are the high and low-frequency limit of the permittivity, respectively. $\Delta \varepsilon=\varepsilon_{l}-\varepsilon_{h}$ is the relaxation amplitude, and $\tau_{\varepsilon}$ is the relaxation time. This model applies for nearly dehydrated micelles $(\omega \simeq 0)$ where the dielectric relaxation has been shown to result from rotational re-orientation of the whole micell and the diffusion of hydrated AOT ion pairs [28, 5].

At larger molar ratio and volume fraction of the critical composition the ploydispersity of the micelles leads to a distribution of micellar radii giving rise to a distribution of relaxation times. Apart from the critical phase transition region the distribution is approximately symmetrid 11 which is formalised by the Cole-Cole model according to [46]

$$
\varepsilon=\varepsilon_{h}+\frac{\Delta \varepsilon}{1+\left(i 2 \pi f \tau_{\varepsilon}\right)^{1-\alpha}} \quad(0<\alpha<1) .
$$

The real and imaginary part separately read [6]:

$$
\begin{aligned}
\varepsilon^{\prime} & =\varepsilon_{h}+\frac{\Delta \varepsilon\left(1+\left(2 \pi f \tau_{\varepsilon}\right)^{1-\alpha} \cos (\pi(1-\alpha) / 2)\right)}{1+2\left(2 \pi f \tau_{\varepsilon}\right)^{1-\alpha} \cos (\pi(1-\alpha) / 2)+\left(2 \pi f \tau_{\varepsilon}\right)^{1-\alpha}} \\
\varepsilon^{\prime \prime} & =\frac{\Delta \varepsilon\left(\omega \tau_{\varepsilon}\right)^{1-\alpha} \sin (\pi(1-\alpha) / 2)}{1+2\left(2 \pi f \tau_{\varepsilon}\right)^{1-\alpha} \cos (\pi(1-\alpha) / 2)+\left(2 \pi f \tau_{\varepsilon}\right)^{1-\alpha}} .
\end{aligned}
$$

The dielectric relaxation mechanism differs from that of smaller dehydrated micelles as the larger micellar radius inhibits rotational re-orientation. According to D'ANGELO et al. 29, the microscopic origin of the polarisation of micelles at $10<\omega<25$ is a fluctuating dipole moment induced by the free diffusion of AOT ion pairs at the water-surfactant interface. This picture also

\footnotetext{
${ }^{1}$ The symmetric distribution of relaxations in WDA has been shown to cross-over to an asymmetric one in approaching the consolute point [78].
} 
holds for the critical composition. Furthermore, an average aggregation consists of several micelles leading to anion exchanges between adjacent micelles which also contributs to the relaxation.

In addition to the considerations of Sec. 2.2.1, it should be noted that the water core radius is large enough for the water to behave as a "free" pseudophase. The additional molecular relaxation of the free water present in the system exceeds the frequency range of this measurement $\left(10^{8}<f<10^{10} \mathrm{~Hz}\right)$. Water is known to exhibit a single Debye type relaxation due to molecular re-orientation, which causes an offset in the DRS data which is related to the water content of the specimen.

Figure 4.4 depicts the real and the imaginary part of the complex permittivity of three representative WDA specimen with and without additional tracers at $T=293 \mathrm{~K}$. The experimental data is approximated by the Cole-Cole model by simultaneous minimisation of both terms of Eq. 4.4 using a nonlinear leastsquare algorithm (full lines in Fig. 4.4). The relaxation is found in the $100 \mathrm{MHz}$ region which is in agreement with previous investigations 29, 78. Firstly, it is noteworthy that the different specimens without additional tracers (open symbols in Fig. 4.4) exhibit significant differences, although the nominal composition and the preparation procedure are identical for all the microemulsions. Secondly, the results for tracer loaded WDA do not show a consistent trend in $\varepsilon^{\prime}$ (this has been verified by additional results not included here). The characteristic frequency $\tau_{\epsilon}$ the peak location of $\varepsilon^{\prime \prime}$, is slightly or even moderately shifted. The variation of $\tau_{\epsilon}$ indicates the distribution of micellar sizes to differ between the specimen which, according to Eq. 2.21, can be translated into differences of $\omega$.

Furthermore, the variation of the relaxation amplitude in $\varepsilon^{\prime}$ is accompanied by an offset of the whole curve which points at a varying water content of the specimen. The relaxation amplitude of the molecular water at $f>1 \mathrm{GHz}$ is accountable for the offset while a change of average micellar size alters the amplitude of the $100 \mathrm{MHz}$ relaxation. The former is not included in the fit which is the reason for the deviation of the Cole-Cole model at higher frequencies close to $f=1 \mathrm{GHz}$. As the variation of $\omega$ is obviously equally strong for specimens based on the same as well as on different stock solutions, no systematic tracer-micell interaction can be deduced from the DRS measurements.

It should be mentioned that a contamination with water alters the criticality of the specimen. The temperature dependence of the mean aggregation depends on the criticality which would be also visible in a shift of the characteristic frequency. An effect which might apply close to the critical point, but which is considered not important at approximately $15 \mathrm{~K}$ below $T_{c}$.

In summary, comparing the different WDA specimen, the influence of tracers turns out to be indistinguishable from constitution variations or effects of contamination. Moreover, the varying water content of the specimen independent of the addition of particles covers possible effects related to the presence of tracers. However, there is no indication of carboxyl latex trac- 

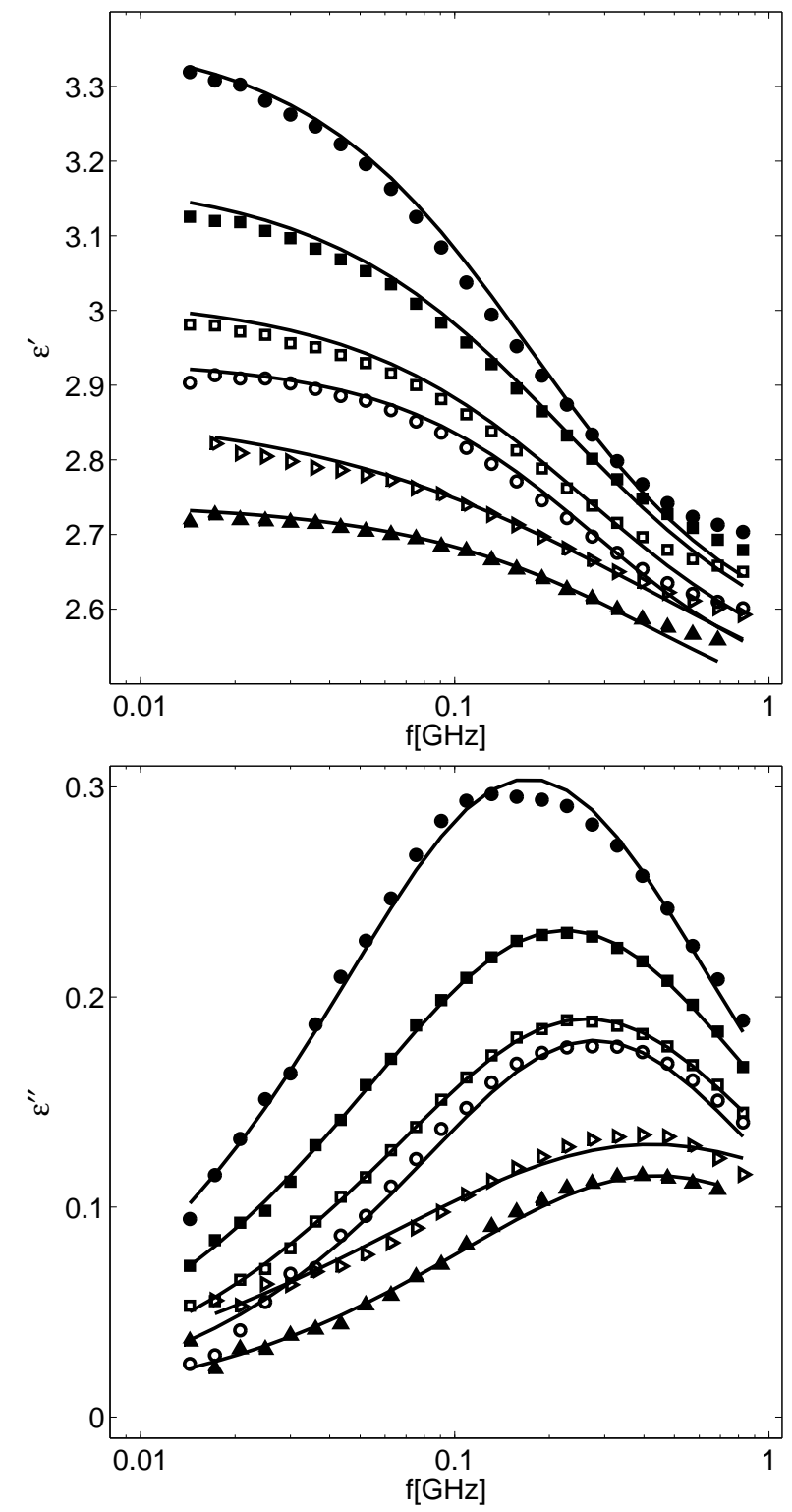

Figure 4.4: The real $\varepsilon^{\prime}$ and imaginary part $\varepsilon^{\prime \prime}$ of the complex permittivity of three different specimens of WDA ( $\omega=40.9, \eta=0.098, T=293 \mathrm{~K})$. The filled symbols indicate additional tracers; corresponding symbols refer to WDA specimens based on the same batch; the full lines represent the best fit to Eq. 4.4.

ers to strongly interact with inverted micelles of WDA. However, the DRS measurements emphasise the sensitivity or instability of the WDA system. During the preparation and the insertion of tracers, exposure to ambient air is 


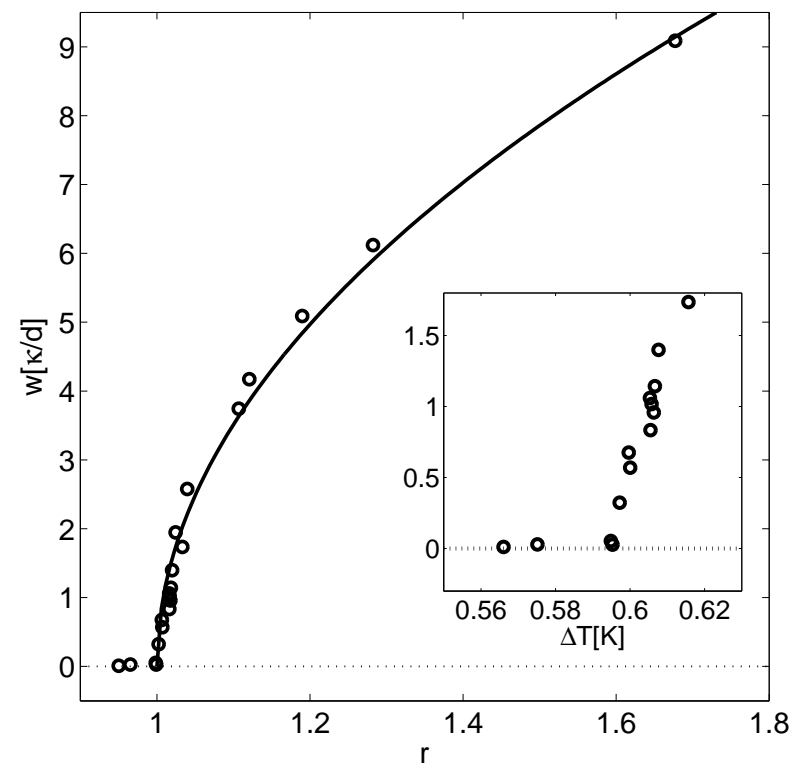

Figure 4.5: The maximum vertical velocity $w$ versus reduced Rayleigh number $r$ for decane and the theoretical curve according to Eq. 4.5 (full line) at $T_{t o p}=298 \mathrm{~K}$. The inset shows a magnification of the data close to $w=0$ where the abscissa depicts the temperature difference. The critical value is $\Delta T_{c}=0.596(5)$.

inevitable and this is considered to be the source of the varying water content of the specimen.

\subsubsection{Convection in Pure $n$-Decane}

Convection in pure decane is considered a reference case for the evaluation of the quality of the experimental design. Figure 4.5 depicts the maximum vertical velocity as a function of reduced Rayleigh number $r$ thereby summarising the results. The critical temperature difference is $\Delta T_{c}=0.596(5)$ corresponding to a critical Rayleigh number of $R_{c}=1741(39)$ which is very close to the literature value of $R_{c}=1719$ at an aspect ratio of the smaller side of the cell of $\mathbb{E}=4$ [99]). Furthermore, the characteristic of the maximum $w$ versus $r$ is found to closely obey the theoretical prediction [79]

$$
w[\kappa / d]=0.87 \sqrt{2} k_{c}^{2}(r-1)^{1 / 2}
$$

and maximum values of both velocity components $u$ and $w$ coincide within the experimental uncertainty. The critical wave number $k_{c}=2.93$ is slightly reduced with respect to the literature value of $k_{c}=3.005$ [99], which is an effect of the containment of the fluid layer by the cell geometry. 
These results clearly demonstrate the suitability of the experimental design. The comparatively low quality of the thermal boundary conditions, i.e. the small Biot number does not impose substantial disturbances. The side-wall forcing from the lateral boundaries has a minor effect, and sufficiently dense tracer distributions are assured. 


\subsection{Binary Fluid Convection}

In this section direct velocity amplitude measurements of molecular, binary ethanol-water mixtures in a narrow convection cell with rectangular shape will be presented and discussed. The specific geometry of the convection cell with an aspect ratio of $\mathbb{E}=1 \times 4 \times 30$ forces convective roll structures to align perpendicular to the longer side, enabling velocity measurements perpendicular to the roll axis. Both positive and negative Soret coupling will be considered giving rise to stationary as well as oscillatory travelling wave convection.

\subsubsection{Stationary Convection}

For the detailed investigation of a positive Soret coupling in a molecular mixture, a $40 \%$ wt ethanol-water binary mixture with a moderate separation ratio of $\psi=0.23$ has been chosen. In the temperature interval of the experiments, the mixture is characterised by a Prandtl number of $\sigma \simeq 27$ and a Lewis number of $L \simeq 0.0045$. The experimental procedure is to initially set the Rayleigh number to $r>1$, starting from a fully developed roll mode in the Rayleigh regime, and then to subsequently lower the Rayleigh number quasi-statically. After each step, the fluid is allowed to relax approximately $20 \tau_{v}$ with $\tau_{v}=d^{2} / \kappa \simeq 103 \mathrm{~s}$. The experimental path is primarily a requirement of the $\mu \mathrm{PIV}$ technique, since tracer settling is minimised retaining a homogeneous distribution. Additionally, the two-dimensional roll state of the Rayleigh regime is stabilised to as low as possible Rayleigh numbers.

Figure 4.6 depicts the maximum velocities $u$ and $w$ versus Rayleigh number $r$ together with the theoretical amplitude for a pure fluid. Beginning at $r \simeq 1.25$ the Rayleigh regime is marked by a single roll mode at a slightly smaller wave number $k=2.7(1)$ as compared to the pure fluid case. Decreasing $r$, the velocity amplitude decreases closely following the theoretical curve. Approximately at $r=1$, the curve has an inflexion point with a decreasing slope thereafter. Subsequently, at $r=0.91(1)$, the convective amplitude suddenly drops as the two-dimensional roll mode loses stability to cross-rolls. The term cross-rolls will be used in its most general sense for structures of two perpendicular roll modes albeit that the accurate nature of the structures (square-like, ordered or disordered) cannot be differentiated based on the two-dimensional measurements. The cross-roll regime is preserved to $r=0.56(2)$, where the vertical motion vanishes over the whole length of the cell, except for the very vicinity of the lateral boundaries, indicating a single large-scale structure to fill the geometry. Extracting the critical temperature gradient for the onset of the Rayleigh mode $\Delta T_{c}^{p}$ by a linear fit to the square of the amplitude in the Rayleigh regime $\left(A^{2} \gg \psi L\right)$, the critical Rayleigh number is $R_{c}=1658(39)$ which matches the literature value within $3 \%$ [99]. This value is used to scale the Rayleigh numbers in the remainder. 


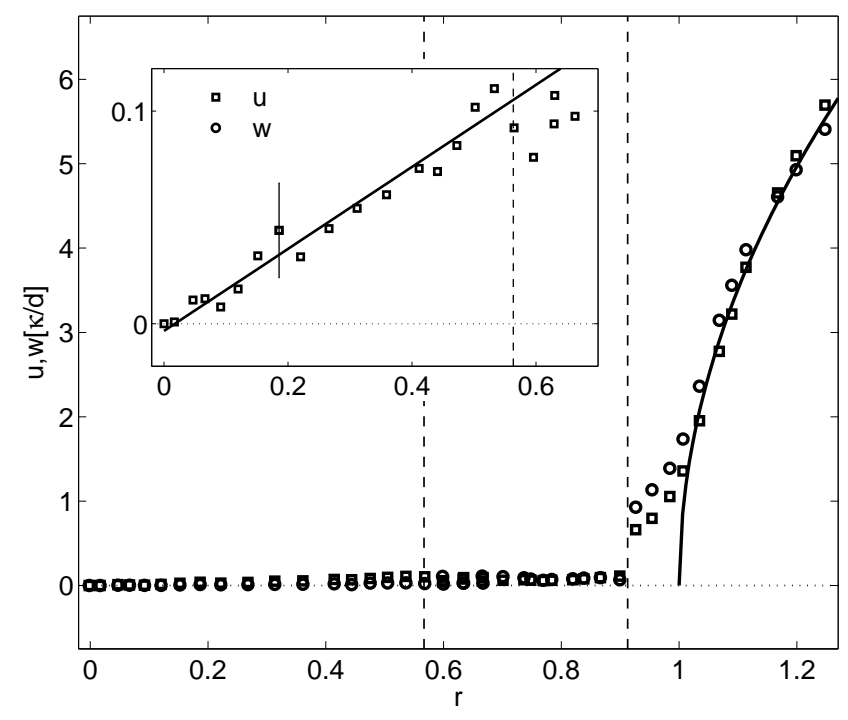

Figure 4.6: The maximum vertical and horizontal velocities $w(\circ), u(\square)$ versus reduced Rayleigh number $r$ for ethanol - water mixture with $\psi=$ $0.23, L \simeq 0.0045$ at $T_{t}=298 \mathrm{~K}$. The inset is a magnification of the $u$-component close to $r=0$. The dashed lines indicate structural transitions from rolls to cross-rolls at $r=0.91(1)$ and cross-rolls to the large-scale structure at $r=0.56(2)$.

\section{The Soret Regime}

In the intermediate regime of cross-rolls between the Rayleigh mode and the large-scale structure, the velocity measurements are not reliable since the structure might have any orientation with respect to the cell centre, i.e. the field of view does not necessarily intersect the region of maximum velocity amplitude. The velocities in this region cannot be considered representative and therefore the data is excluded from the quantitative analysis.

The observation of the large-scale structure is in agreement with the linear stability analysis (cp. Sec. 2.1.2) since the critical wave number is predicted to vanish for $\psi$ exceeding $\psi_{\infty}=131 L /(34-131 L) \simeq 0.02$ 49. In this regime, $u$ is found to linearly decrease with decreasing $r$ (Fig. 4.6). A linear fit to the velocity yields $r_{c}=0.013(7)$ which is in reasonable agreement with Eq. 2.12 leading to $r_{c}=0.0085$. Note that the time scale for the establishment of the large-scale structure is obviously the vertical diffusion relaxation time $\tau_{v, D} \simeq$ $6 \mathrm{~h}$. The horizontal diffusion relaxation time $\tau_{h, D}$ would be approximately one day. Consequently, the experimental relaxation was extended to $\tau_{v, D}$ in this region. 
An important parameter for the characterisation of small amplitude convection is the initial slope of the heat transport, the Nusselt number $N=h d / \lambda \Delta T$ which can be estimated based on the slope of the velocity amplitude. In the large-scale structure regime, the slope $S_{u}=\partial u / \partial r$ is found to be $0.108(7)$. Comparing this value to that of the Rayleigh mode $S_{u}^{p}=20.193(5)$, $S_{u} / S_{u}^{p}=0.0053$ is an order of magnitude smaller than the velocity amplitude measured by Laser Doppler Velocimetry (LDA) in a comparable system with $\psi=0.35$ 100. Taking into account that the Nusselt number for pure fluid convection between low thermal conductivity boundaries is known to be proportional to the square of the wave number, a crude estimation yields $\partial N / \partial r=\mathrm{O}\left(10^{-3}\right)$. This value is an order of magnitude larger than the prediction for an infinite fluid layer, $S=0.625 \psi L=6 \cdot 10^{-4}$ which is an effect of the containment 53. In the same spirit, the ratio of the slopes in $N$ can be estimated as $S_{N} / S_{N}^{p}=\mathrm{O}\left(10^{-5}\right)$ highlighting the extreme inefficiency of the heat transport in the Soret regime.

\section{The Transition to the Rayleigh Mode}

In order to quantify the development of the flow field in the transition regime at $r \simeq 1$, the lateral velocity profiles are considered. The $w$-profiles are extracted at the mid-plane of the cell $(z / d=0)$ whereas $u$ is taken at the vertical position of maximum amplitude at $z / d= \pm 0.28$. The profiles are described by a Fourier series according to Eq. 2.17 retaining two modes only 9. Due to the spatial symmetry condition for stationary rolls $w_{n}(z)=(-1)^{n+1} w_{n}(-z)$ around the mid-plane of the cell, the even modes of $w$ vanish and the characteristic of the profile is given in terms of $w_{1}$ and $w_{3}$. The velocity data is approximated using a non-linear least-square algorithm and the practicability of the two-mode approach has been verified by the vanishing of higher order modes within the uncertainty of the fit.

In Fig. 4.7 the Fourier mode amplitudes are presented versus $r$ together with the theoretical curves for a pure fluid. Strikingly, $\left|u_{1}\right|$ and $\left|u_{2}\right|$ are in satisfactory agreement with the pure fluid predictions in the Rayleigh regime $r>1$. In the closer vicinity of the transition to the Soret regime $r \simeq 1,\left|u_{2}\right|$ rises towards $\left|u_{1}\right|$ for decreasing $r$. The first mode of the vertical velocity component $\left|w_{1}\right|$ is smaller than the theoretical line in the region $r \geq 1.15$. $\left|w_{3}\right|$ is considerably larger than the prediction and increases monotonically for decreasing $r>1$. The growth of $\left|w_{3}\right|$ indicates the growing non-linearity of the $w$-profile, i.e. the basal mode flattens $\left(\left|w_{1}\right|\right)$ while the rise of the second mode reflects the steepening of the maximum in the transition to the Soret regime, see Fig. 4.8 As the thermal driving weakens, the core region of the rolls slows down, while the convective fluid motion is increasingly restricted to the interface between adjacent rolls. Additionally, the disagreement of $w_{1}$ with the theoretical value results from the wave number which decrease from $k=2.7(1)$ at $r=1.16(1)$ to $k=2.3(1)$ at $r=0.92(1)$. Both velocity components are related 


\subsection{Binary Fluid Convection}
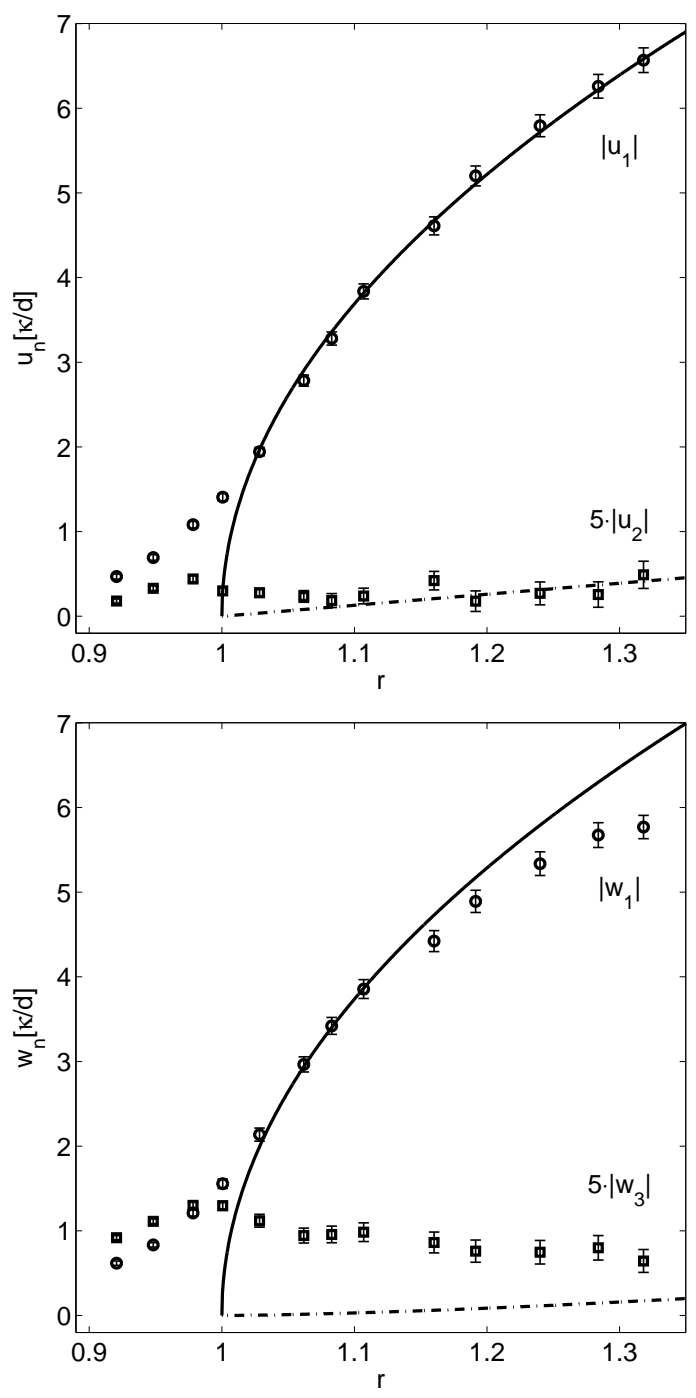

Figure 4.7: The lateral Fourier mode amplitudes in the transition region between the Rayleigh and Soret regime; the error bars indicate the uncertainty of the approximation.

by the continuity equation which, for a single roll, can be written as

$$
\int_{0}^{d / 2} u(0, z) d z=\int_{0}^{\Gamma / 2} w(y, 0) d y
$$

with the wave length $\Gamma$, where the origin $(0,0)$ is located at the centre of the roll [94. A first order approximation of Eq. 4.6 yields $u^{\max } \simeq \frac{\pi}{k_{c}} w^{\max }$ which shows $w^{\max }$ to be smaller than $u^{\max }$ for not to small deviations from $k_{c}^{p}$. Identifying the maximum values with the first modes, the deviation of $\left|w_{1}\right|$ 

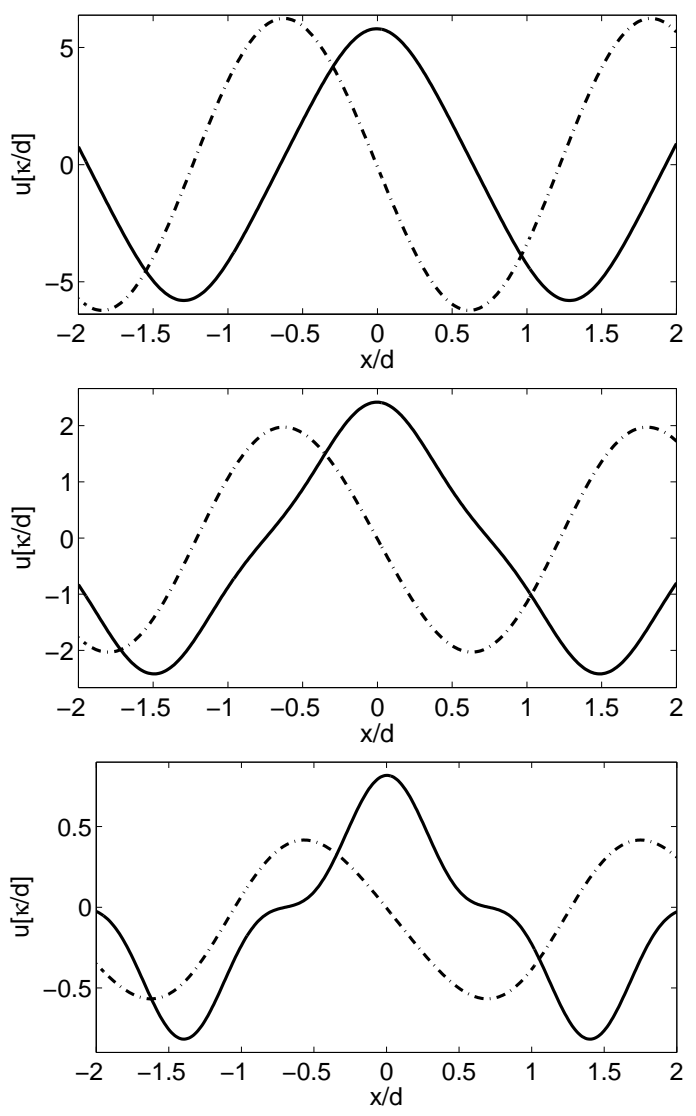

Figure 4.8: The lateral $u$ (dash-dotted) $w$-profiles (full line) approximated by the first two Fourier modes $r=1.11(1)$ (top), $r=1.00(1)$ (centre), and $r=0.92(1)$ (bottom).

from the theoretical curve is due to the mismatch of the wave number with the model value, $k=k_{c}^{p}$.

The intersection of the maximum values of $u$ and $w$ at $r \simeq 1$ (visible in Fig. 4.6) is a result of the combination of decreasing the wave number and the steepening of the $w$-profile increasing the maximum with respect to $u$.

In order to identify oscillations or possible unsteadiness of the structures in the transition to the Soret regime, POD is employed which covers time series of approximately $\tau_{v}$. Figure 4.9 depicts the eigenvalues of the first three modes in the Rayleigh number interval of the transition. The basal roll structure of the rolls is covered by the first mode which at $r>1$ represents more than $90 \%$ of the energy of the flow while the modes two and three are found to be unstructured with an energy content of the order of a one percent, still considerably above the remaining modes (Fig. 4.10). The eigenvalue of the first mode decreases considerably at $r<1$ to approximately $65 \%$ but the time 


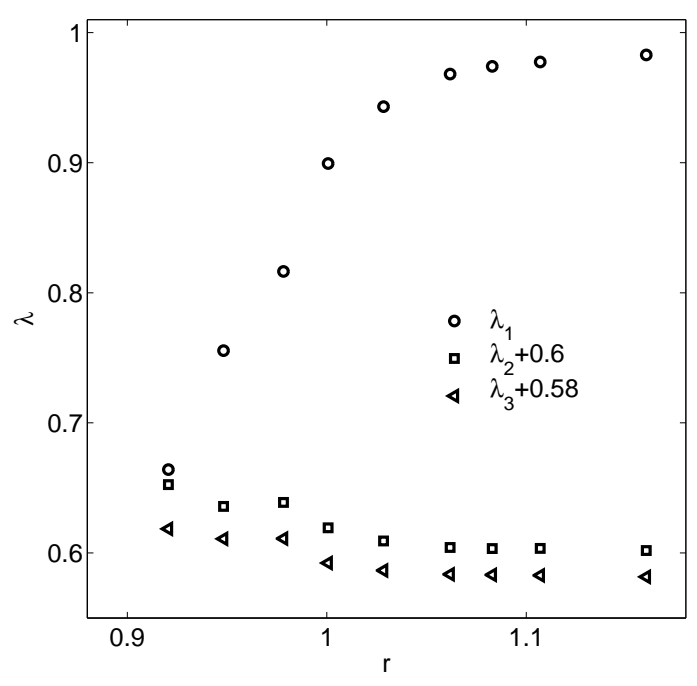

Figure 4.9: The eigenvalues $\lambda$ of the first three POD mode versus $r$ in the transition regime.

evolution of the mode (given by the time evolution coefficient $\zeta_{1}(r=0.92)$ ) does not indicate intermittent or oscillatory behaviour. Simultaneously, the energy gain of the second and third mode is moderate and most of the energy is obviously transfered into an accession of all modes.

The modes two and three are not identified with a flow structure but essentially represent a fraction of non-random measurement noise. The random fluctuations can be anticipated to be distributed equally over all the modes. Note that the second and third mode is apparently not divergence free. POD modes in general are not restricted to be divergence free but the two-dimensionality of the flow field is an additional argument in advance of the measurement noise interpretation.

In summary, the experiments indicate neither oscillations nor intermittent effects in the transition between the Rayleigh and the Soret mode. The transition is stationary, characterised by transfer of the roll structures to plume-like structures and the two dimensional roll mode breaks off when the internal re-mixing effect becomes insufficient to stabilise the core region.

\section{Discussion}

The pattern formation in the transition regime depends on the experimental path, i.e. increasing or decreasing $r$. Therefore, the present experiments should be discussed by comparison to visualisation experiments by Moses \& STEINBERG [87, 89] who have investigated an identical mixture in a rectangular cell of comparable dimensions to the presently used $(1 \times 4 \times 12$ with $d=2.98 \mathrm{~mm}$ ) advancing $r=1$ from below. These authors observed the cross- 

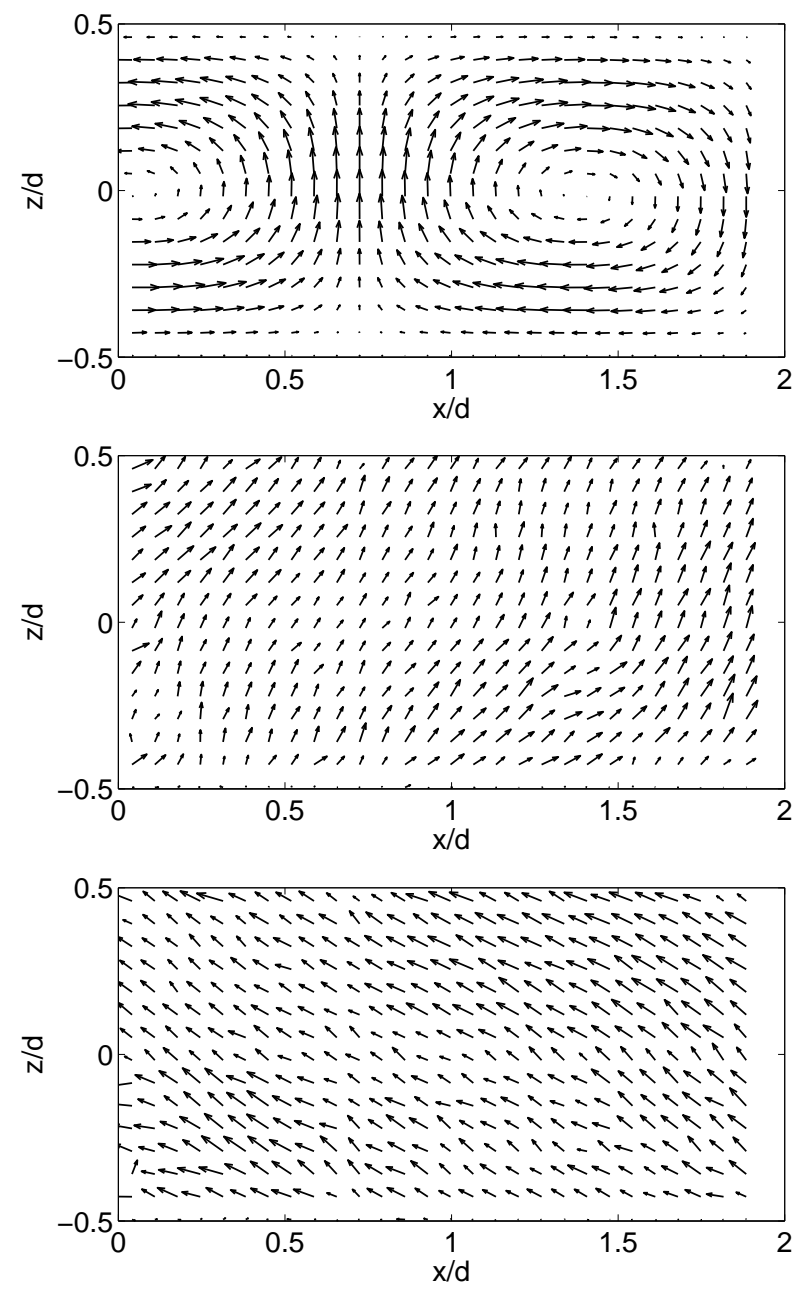

Figure 4.10: The velocity field representation of the eigenmodes one to three of the decomposition at $r=1.16(1)$.

roll or square-like patterns in a very narrow $r$ interval beginning at $r=0.95$. The transition to rolls has been indicated at $r \simeq 1$ and stable rolls (i.e. the Rayleigh mode) have been found at $r \geq 1.07$. According to the present results it is obvious that a fully developed Rayleigh mode has a stabilising effect in the transition region where square structures are also stable. In the absence of coherent two-dimensional rolls, the reduced re-mixing preserves the threedimensional pattern in combination with concentration injections from the boundaries of the convection cell. Additionally, the narrow annular cell of the experiments discussed here has to be regarded as an auxiliary stabilisation of the Rayleigh mode as oscillatory states are suppressed by the quasi-onedimensional geometry. The cell geometry effectively prevents the evolution of 


\subsection{Binary Fluid Convection}

modes parallel to the long side and, therefore, preserves the two-dimensional roll state which is also confirmed by [89. In summary, the pattern transition at $r \simeq 1$ is hysteretic and the systems' behaviour can be regarded as an imperfect bifurcation, where the concentration enhancement of the thermal destabilisation represents the imperfection imposed onto the Rayleigh-Bénard instability. However, the extent of the hysteresis appears to be rather moderate $(0.92 \leq r \leq 1.07)$.

It is instructive to compare the experimental findings to the semi-analytical model given by HOLLinger [50] as this particular model implies two-dimensional rolls only. The description is based on an extension of the Lorentz model for convection in one-component fluids to binary mixtures based on a truncated Galerkin expansions of the fields. The velocity field is approximated by one mode at constant wave number $(k=\pi)$ and no-slip boundary conditions. Although the model has been initiated to capture the bifurcation behaviour and convective states of binary fluids with $\psi<0$, the description of stationary states also holds for a positive Soret coupling. Assuming stationary convection the following relation for the order parameter and the convection amplitude have been given.

$$
r\left(X^{2}\right)=\frac{1+X^{2}}{1+\frac{11}{5} a \frac{\psi}{L} \frac{\left(1+X^{2}\right)\left[1+\frac{25}{99} a\left(\frac{X}{L}\right)^{2}\right]}{1+\frac{185}{36} a\left(\frac{X}{L}\right)^{2}+\frac{625}{648} a^{2}\left(\frac{X}{L}\right)^{4}}}
$$

with

$$
a=\frac{9 \pi^{2}}{128} \quad \text { and } \quad X=\frac{8}{5 \pi^{2}} w .
$$

The amplitude of $w$ is slightly larger than the one of the model for the onecomponent fluid given by Normand 94. According to the present measurements the latter is in excellent agreement with the values measured in pure decane and in the Rayleigh mode of the binary mixture. Thus, the amplitude in Eq. 4.9 is adjusted to this value. The criterion for stationary stability of the quiescent conducting state of the model is given as:

$$
r_{c}=\frac{1}{1+\frac{11}{5} a \frac{\psi}{L}} .
$$

Inserting $\psi$ and $L$ yields $r_{c}=0.013$ which perfectly matches the experimental result. Figure 4.11 depicts the model of Eq. 4.7 by comparison with the measured $u$ component. The model nicely agrees with the Rayleigh mode amplitudes and the inflexion in the transition regime. The model incorporates the Soret effect in boundary conditions and discards concentration gradients in the bulk. The argument for this simplification is that the small size of the Lewis number ensures transport by diffusion to be small compared to advection in presence of the Rayleigh mode. The agreement of the present experimental data with this simplified description shows the concentration boundary layers 


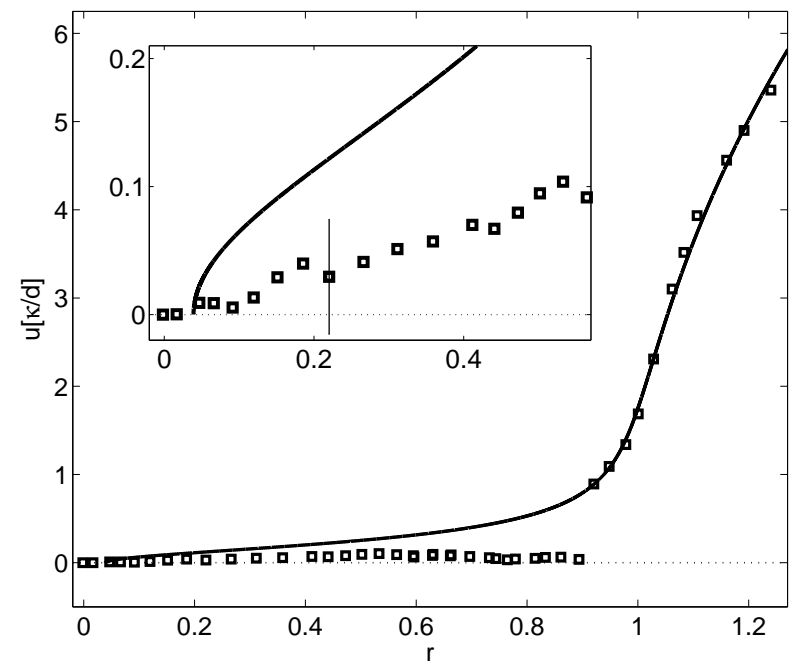

Figure 4.11: Comparison of the velocity amplitude and the model of Eq. 4.7 The inset clarifies the mismatch of the model and the experimental data for the large-scale structure and depicts a representative error bar.

to be thin and not to grow significantly as $r$ decreases, as long as the roll state is stable. However, the concentration supported convective motion is restricted to the relatively thin separatrix between adjacent rolls and transport by diffusion is too small to broaden the area. As a result, the lateral amplitude profiles steepen and the core region of the rolls decouples from the up- and down-flow regions of the separatrix (cp. Fig. 4.8). The internal mixing in the cores decreases until advection becomes comparable to diffusion. Therefore, the core region itself becomes susceptible to the Soret effect destabilising the two-dimensional roll state which leads to the transition to cross-rolls.

In presence of three-dimensional flow structures, the model fails. However, the comparison with the large-scale structures is interesting because it clearly shows the three-dimensional nature of the flow as the measured amplitude is smaller and decreases more weakly than the artificial roll solution for $r \rightarrow 0$.

Another interesting observation in comparison to the shadowgraph visualisation of [89] is that the Soret induced cross-roll structures are extending to Rayleigh number as low as $r=0.56(1)$ (see Fig. 4.6). Moses \& Steinberg 89] have been able to discern a large-scale structure in thinner cells of varying aspect ratio distinctly above the predicted $r_{c}$ (at $r=0.16$ ). As a result of the limited sensitivity of the shadowgraph, the onset has not been observed and $r_{c}$ has been mismatched. However, the development of the smaller-scale pattern 


\subsection{Binary Fluid Convection}

on top of the large-scale one has been reported to be completed at $r=0.96$. Thus, the very low value of $r$ for the transition between the large-scale structure and cross-rolls found in the present experiment has to be attributed to the experimental path. Decreasing $r$, advection promotes the rising of fluid from the concentration boundary layer by means of entrainment. At the same time the concentration boundary layer remains comparatively thin due to the mixing effect of the cross-rolls. This state terminates when concentration gradients equilibrate faster than the time scale of the entrainment motion. Then, the system falls back into its natural time scale $\left(\sim L^{-1}\right)$ and the large-scale structure develops from the concentration expansion over the whole cell. Hence, this stage of pattern formation shows a much larger hysteresis than the transition to the Rayleigh mode. However, it should be noted that measurements along decreasing $r$ provide the opportunity to bypass the diffusion time scale of the system, as long as cross-rolls are stable.

A comment should be added on the initial wave numbers of the Rayleigh mode. It is conspicuous that $k$ is considerably smaller than expected. The homogenisation of the concentration field suggests the convective structure in this regime to be indistinguishable from that in a pure fluid. On the other hand, the relatively small mismatch of $\Delta T_{c}^{p}$ with the theoretical value and the excellent agreement of the amplitude with the pure fluid predictions contradict an explanation in terms of imperfections of the thermal boundary conditions.

Furthermore, if the deviation of the wave number were due to the fairly small Biot number of the cell, the wave number would not be expected to increase in the Rayleigh regime close to $r=1$. Both the amplitude and the critical Rayleigh number should be noticeably altered if the thermal boundaries were the reason for the deviation of $k$ 94. In summary, this point cannot be elucidated satisfactorily because both the convective amplitude and the critical Rayleigh number do not exhibit considerable deviations from the pure fluid reference.

Reviewing the present experimental findings, the bifurcation behaviour is in agreement with the theoretical predictions. The quantitative determination of the critical Rayleigh number $r_{c}$ agrees with the stability analysis prediction. The transitions between the different patterns is stationary and exhibit hysteresis resulting from advective homogenisation of the concentration field.

\subsubsection{Travelling Wave Convection}

For the investigation of travelling wave convection, a $25 \%$ wt mixture of ethanolwater was chosen with a separation ratio of $\psi=-0.087$ [45, 63] at $T=300 \mathrm{~K}$. The Prandtl number is $\sigma \simeq 18$ and the Lewis number is found to be $L \simeq 0.0061$. The experimental procedure was to drive the system into the state of $\mathrm{TW}$ at $r>r_{c o}$ initiating a fully developed travelling wave state and lowering the Rayleigh number $r$ in small steps thereafter until the transition to conduction at $r_{s n}$ was reached. Subsequently, the critical Rayleigh number, $r_{c o}$, for the 


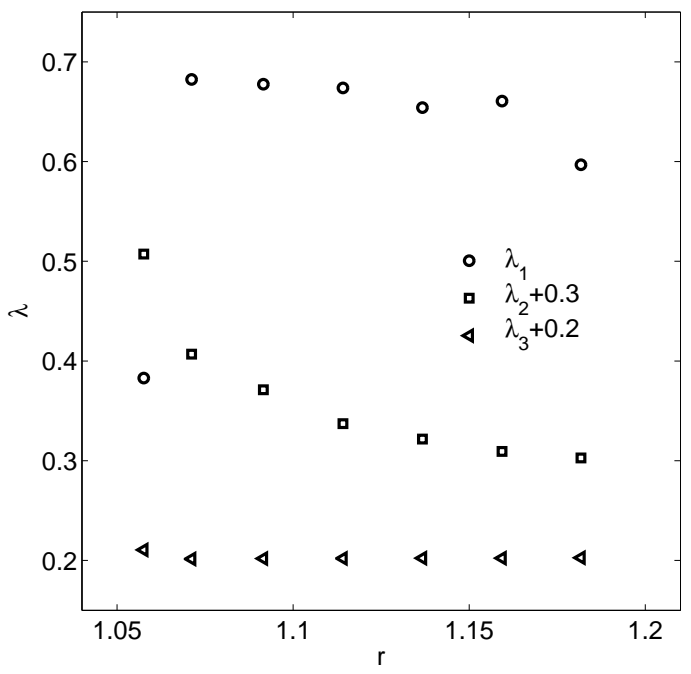

Figure 4.12: The eigenvalues $\lambda_{i}$ of the first three POD mode versus $r$ of TW convection in a $25 \%$ wt ethanol-water mixture with $\psi=-0.087$.

onset of TW convection was determined. In approaching $r_{c o}$ from below, the fluid was allowed to relax approximately $50 \tau_{v}$ while the relaxation time in between travelling wave states was $30 \tau_{v}$ with the vertical thermal relaxation time $\tau_{v} \simeq 83 \mathrm{~s}$.

This experiment has to be regarded as a borderline case for the application of $\mu \mathrm{PIV}$ because the moving roll structure does not allow for an effective averaging. Note that the ensemble correlation evaluation could be realised in a co-moving frame but this would narrow the resulting velocity field proportional to the phase velocity of the TW. Therefore, the data is evaluated by means of a two-mode reconstruction based on the POD (Fig. 4.13). However, the noise level is found to be substantially larger compared to stationary convection due to the separation of the basal convection structure into two POD modes. The POD evaluation requires "dense" tracer images. In the present case approximately half the measurement data had to be discarded which is the explanation for the comparatively few data points of the results.

Figure 4.12 presents the eigenvalues of the first three POD modes. Mode one and two cover the structure information whereas the third represents the most energy rich mode of the background. Modes three and four are comparable to modes two and three of the $40 \% w t$ mixture and might be regarded as the nonrandom measurement noise according to the argumentation of the preceding section. The energy content of the first mode varies between 60 and $70 \%$ while the second one contains up to $12 \%$ of the total energy, depending on the frequency $\omega$ of the TW. The first and second mode are of similar morphology, shifted by half a wavelength against each other. In the limit of large enough 

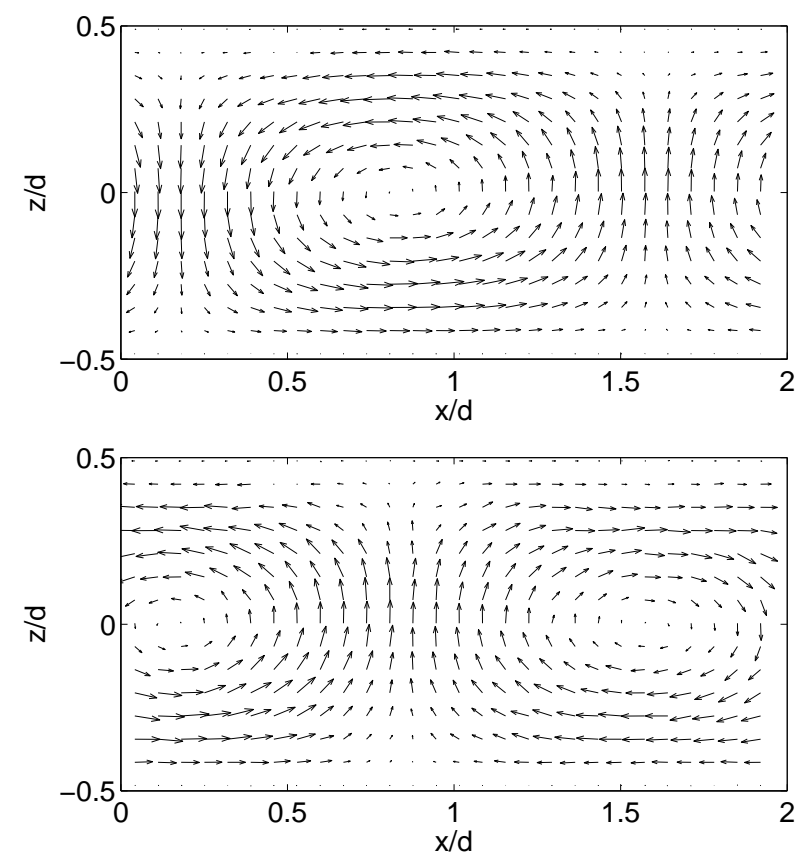

Figure 4.13: The velocity field representation of the first and the second POD eigenmode of the flow at $r=1.08(2)$.

image time series the eigenvalues of both should become equal, forming a mode pair. Otherwise, the increasing TW frequency results in an increasing modulation of the flow structure, due to the limited recording time. Therefore, the energy content of the second mode rises with increasing $\omega$. The noise level, represented by the third and higher order modes, remains constant as long the TW state persists.

Increasing $r$, the conductive state loses stability at $r_{c o}=1.13(5)$ (dotted line in Fig. 4.14), which agrees with Eq. 2.13, yielding 1.094 within 4\%. Subsequently, lowering $r$ leads to a TW state at decreasing amplitude and increasing frequency. The transition to conduction is found at $r_{s n}=1.03(4)$ via a state of confined TW which will be addressed separately below. Figure 4.14 depicts the first two modes of the lateral Fourier decomposition of the TW state for both velocity components. The vertical component $w$ is taken at mid-height and $u$ is extracted at its maximum amplitude position which is the same as for the stationary case $(z / d= \pm 0.28)$. Note that the inversion symmetry of the $w$-profile is broken, leading to $n=2$ being the second mode in $w .\left|u_{1}\right|$ and $\left|w_{1}\right|$ are found to be closely below the pure fluid reference in agreement with twodimensional numerical simulations $\left[9\right.$. The slightly larger deviation of $\left|w_{1}\right|$ has to be attributed to the wave number which, within experimental uncertainty, is constant for the Rayleigh numbers measured, $k=2.5(2)$. The small value 

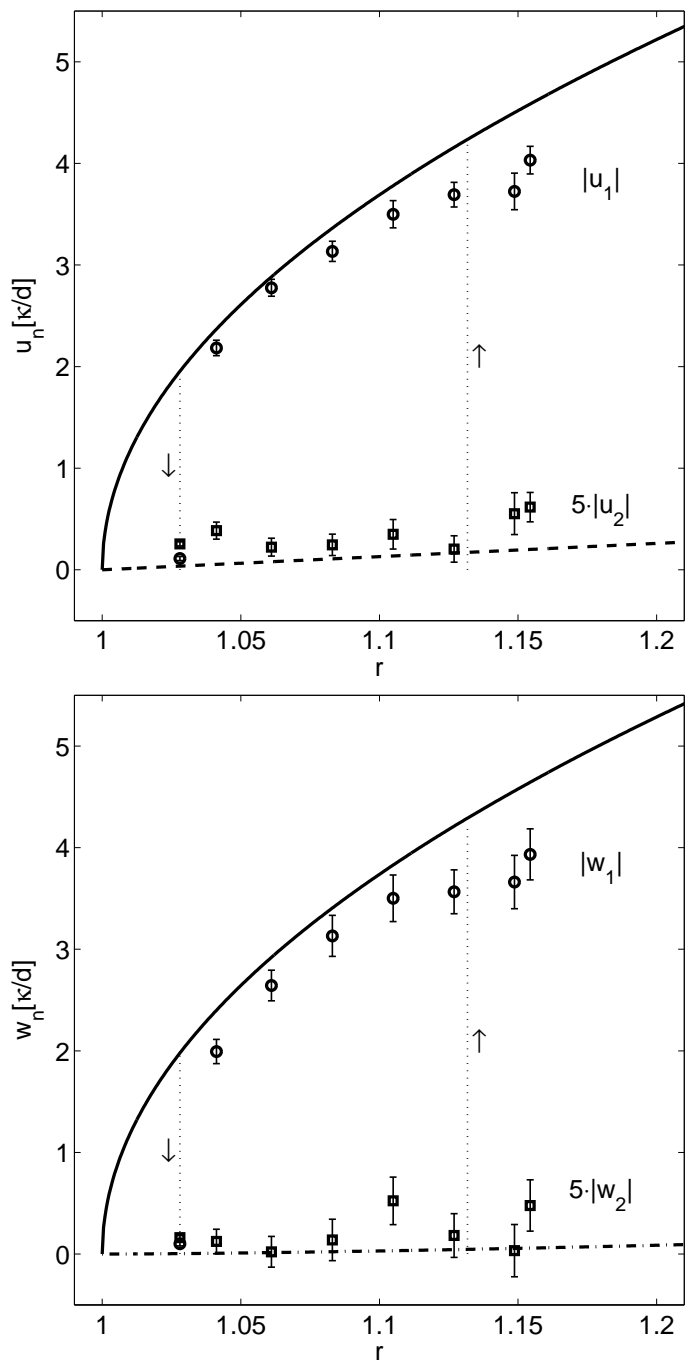

Figure 4.14: The first and second Fourier mode amplitude of $u$ and $w$ in the TW state of the $25 \% w t$ ethanol-water mixture with $\psi=-0.087$, $\sigma \simeq 18$, and $L \simeq 0.0061$; the error bars denote the uncertainty of the approximation.

of the wave number is unexpected as the TW state characteristics are known to closely accord the pure fluid reference suggesting $k_{c}^{p}=3.005$ 99. However, the source of this deviation, similar to that of the stationary Rayleigh mode, has not been resolved.

The second mode exhibits considerable scatter in both velocity components as a result of the increased noise level of the measurements.

The frequency of the TW is determined directly from the zero-crossing of 


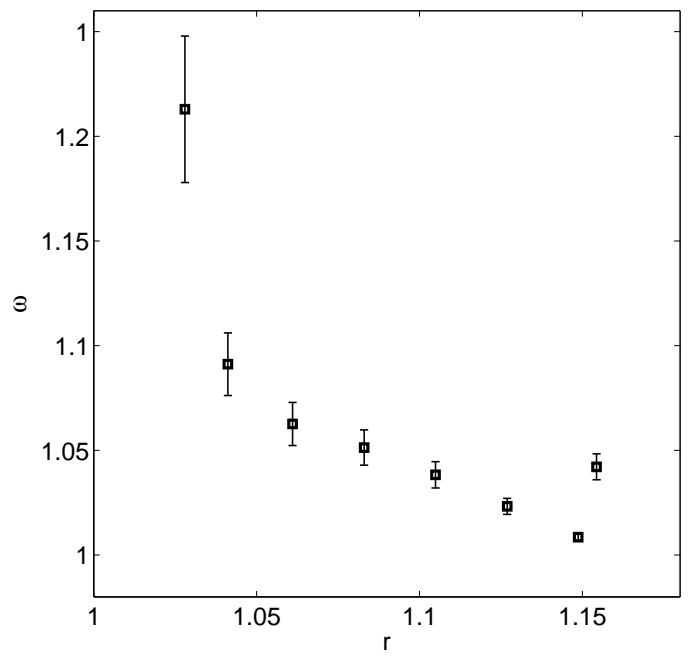

Figure 4.15: The frequency of the TW $\omega$ versus $r$.

the lateral velocity profiles at mid-height. Normalised with the predicted Hopf frequency $\omega_{H}=6.349$ according to Eq. 2.14, the measured frequency of the TW at onset $r_{c o}$ is approximately $0.05(1) \omega_{H}$ which increases to about $0.34(2) \omega_{H}$ at $r=1.03(5)$. Both values nicely agree with the results of numerical simulations 9 .

In summary, the current measurements do not indicate considerable differences from the theoretical predictions for the stationary Rayleigh mode. The underlying explanation is that the concentration field is confined to the very thin boundary layers and the efficiency of mixing in the bulk of the Rayleigh mode masks the binary nature of the fluid. These findings, however, confirm the results of numerical simulations 9 .

\subsubsection{Confined Travelling Wave Convection}

At the smallest measured Rayleigh number the homogeneous TW state vanishes in approximately half of the cell giving rise to a confined travelling wave which is commonly called a blinking state [55, 56]. It should be noted that $r=1.03$ (4) is identified with $r_{s n}$ since it is the lowest $r$ for which TW have been observed in the cell centre.

This confined state is characterised by the alternating emergence and disappearance of confined TW on both sides of the convection cell. The term "blinking" may be deceptive because the dynamics of the structure can be very slow. It is well known that spatially confined blinking TW can be stable in binary mixture convection in narrow finite geometries of rectangular shape [86. The stability of a TW mode only partly extending into the fluid layer due a competition, i.e. destructive interaction of two counter-propagating 

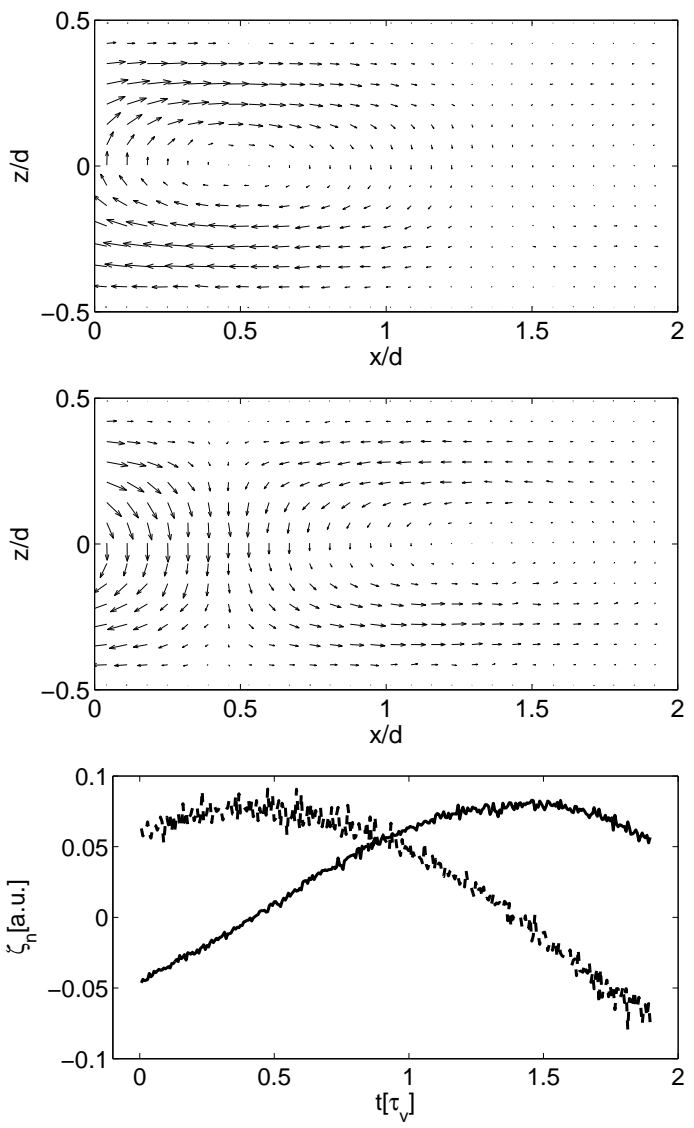

Figure 4.16: The flow field representation of first and second POD mode of the confined TW state at $r=1.03(4)$ (top and centre) and the time evolution coefficients $\zeta_{i}$ for the two modes (bottom).

TW modes, reflexions at the lateral boundaries and back-flow in finite geometries [88. In response to propagating $\mathrm{TW}$ in finite geometries fluid has to be transferred in the opposite direction due to mass conservation which is referred to as back-flow. A theoretical description of the confined TW has been given by CROss 25], considering two left and right travelling waves in the weakly nonlinear regime by means of two coupled amplitude equations of the form of Eq. 2.15. The stability of confined states is identified with a threshold of the non-dimensional group velocity of the TW modes $\bar{s}>2$ in a two-dimensional finite geometry with reflexions at the lateral boundaries. The group velocity is proportional to $\bar{s} \sim(r-1)^{-1 / 2}$, i.e. $\bar{s}$ grows for decreasing Rayleigh numbers $(r \rightarrow 1)$ leading to confined states.

The confined TW state was observed at its border to the neighbouring fluid which is particularly interesting focusing on the back-flow and the convec- 

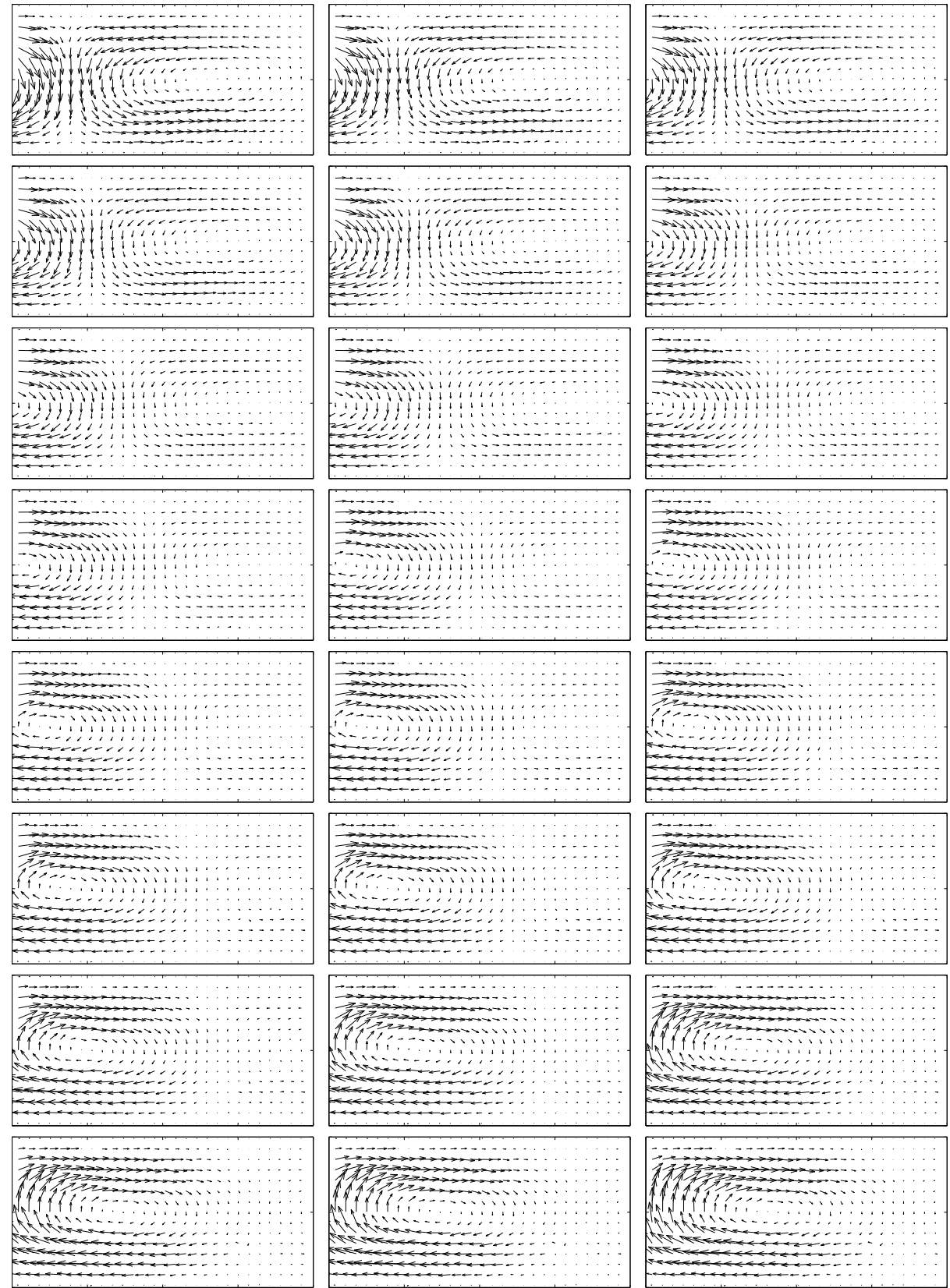

Figure 4.17: Time series of the two-mode approximation of the velocity field comprising the boundary of a confined TW state at $r=1.03(4)$ (from left to right and top to bottom); the time separation of the fields is $0.24 \tau_{v}$. The velocity fields cover the layer depth $d$ and the lateral extent is $2 d$. 


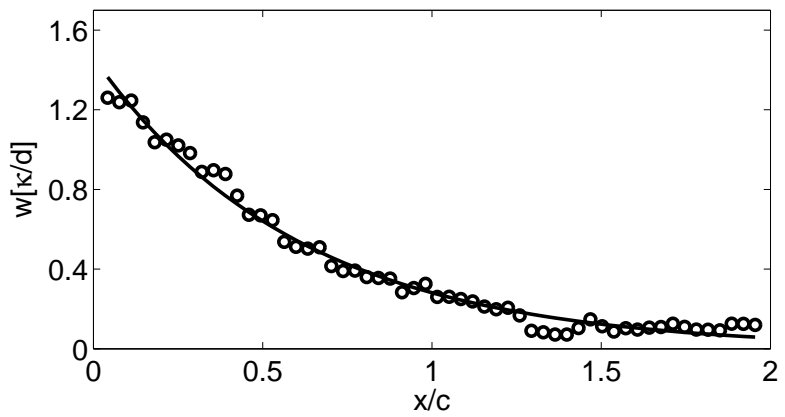

Figure 4.18: The maximum vertical velocity, $w$, versus lateral position, $x$, extracted at $z / d=0$ from the two-mode approximation depicted in Fig. 4.16, The solid line represents exponential decay of the form $w(0, x) \sim e^{-1.6 x}$.

tion amplitude decay at the front to the conductive state. The confined TW state was observed to be stable over several vertical relaxation times $\tau_{v}$ and the front location remained approximately constant during the measurement. Figure 4.16 depicts the time evolution coefficients of the first and second POD mode in addition to the flow field representation of the modes. Both modes represent the same structure at different times . In order to clarify the evolution of the front area Fig. 4.17 shows a sequence of the two-mode approximation of the flow field. The roll structure enters the field of view from the left and moves inwards. Initially, there is a region of down-flow with long ranging branches of lateral motion bordering the confined TW to the right. This down-flow weakens, broadens and ultimately vanishes as the TW advance (this process is not fully completed at the end of the time series). The modes exhibit considerable divergence which indicates the flow to be perpendicular to the field of view in the front region.

The structure evolution at the front can be understood as increasing suppression of the advancing roll structure as an effect of interference with counterpropagating, reflected modes. The horizontal branches close to top and bottom are found to be comparably pronounced which leads to the conclusion that the flow in the immediate front region is perpendicular to plane of the measurements leading to the divergence in the fields of Fig. 4.17. This observation implies back-flow at cell boundaries in $y$-direction, i.e. at the boundaries perpendicular to the roll axis and not close to top and bottom of the cell. Shadowgraph visualisation of confined TW in a comparable geometry have shown that the TW axis is slightly bowed towards the direction of advance [86] which agrees with the picture of the back-flow derived from the present measurements.

As the vertical motion decreases, fluid from the conduction region enters 


\subsection{Binary Fluid Convection}

the roll structure altering the concentration distribution inside the decaying roll. The internal mixing is reduced and thereby the adverse concentration gradient is transfered into the structure damping convective motion. The time series shows the amplitude $w$ of the down-flow region to rapidly decrease while travelling trough the field of view. Adopting the two-dimensional model of [25] the decrease of the vertical velocity amplitude within the front region can be assumed to be exponential which is verified by the experimental data based on the two-mode approximation. The maximum vertical convection amplitude is approximated by $w(0, x) \sim e^{-1.6 x}$ (the solid line in Fig. 4.18).

It should be noted that the confined TW state is remarkably different from localised travelling waves which preserve the individual rolls while travelling through the cell. These patterns have been shown to be stable due to a selfsustaining mixing effect at their front that drives the fluid from conduction to convection and vice versa at the tail [55]. These structures do not imply reflexions to be stable.

Below the confined TW state, small amplitude localised TW usually consisting of two roll structures are observed at the left and right lateral boundaries of the cell which persist for $r<r_{s n}$. Additionally, fluctuations were observed in the bulk in between the localised structures. Due to the relatively large sidewall forcing already indicated in Fig. 3.3, it is assumed that these structures mainly result from the temperature inhomogeneity at the walls. The vertical temperature gradient is more weakly directly at the wall which reduces the adverse concentration gradient leading to wall-sustained TW.

Finally, it should be noted that an attempt to quantify fluctuations below the onset of convection remained without success. The loss of correlation of successive fields rules out evaluation at the necessary, low sampling frequencies $(0.1 \mathrm{~Hz})$. The de-correlation of the particle images for larger inter-framing times due to random motion with respect to the depth of field imposes the dominant intrinsic measurement limitation. 


\subsection{Velocity Measurements of Convection in a Microemulsion}

In this section several compositions of WDA will be considered by means of direct velocity amplitude measurements in the centre of the narrow annular cell also used for the ethanol-water mixtures of the preceding section. The results will be discussed in comparison to the molecular binary mixtures and the colloidal suspension analogy introduced in Sec. 2.3.2.

\subsubsection{Convection in Microemulsions}

The experimental path is similar to that of the preceding sections 4.2 .1 and 4.2.2 The system was initially set to a distinct roll mode, subsequently lowering $\Delta T$ in small steps and acquiring $\mu \mathrm{PIV}$ data after relaxation for $\tau_{r} \simeq 3600 \mathrm{~s}$.

It was chosen to investigate three different mixtures of the compositions given in Tab. 4.1 incorporating meso-structures of different size. Compositions 1 and 2, the water-less micellar solution and the system containing a tenth of the water fraction of the critical mixture, are directly comparable since the differences of volume fraction $\eta$ and molar ratio $\omega$ is approximately $20 \%$ and both systems are known to be dispersions of individual micelles without further aggregation at $T=298 \mathrm{~K}[29$. The third specimen is of critical composition (see App. 1.3). Estimating the concentration relaxation based on the Stokes-Einstein relation (Eq. 2.26) the diffusion coefficients are found to be two orders of magnitude below typical values for molecular mixtures yielding relaxation time scales of the order of days. Clearly, such extreme relaxation times cannot be met within the experimental realisation. Furthermore, the size of the micellar structures of the microemulsion is rather comparable to nano-particle dispersions than molecular binary mixtures.

\begin{tabular}{c|ccccc} 
no & $\omega$ & $\eta$ & $R_{w}[\AA]$ & $R_{h}[\AA]$ & $D\left[\mathrm{~m}^{2} / \mathrm{s}\right]$ \\
1 & 0 & 0.027 & 0 & 19 & $1.498 \cdot 10^{-12}$ \\
2 & 4.08 & 0.032 & 8 & 25 & $1.195 \cdot 10^{-12}$ \\
3 & 40.8 & 0.073 & 23 & 67 & $0.539 \cdot 10^{-12}$
\end{tabular}

Table 4.1: Composition and meso-structure parameters of the different WDA specimens; $D$ is estimated based on $R_{h}$ according to Eq. 2.26

The onset of convection is oscillation-free and the initial roll states of all three specimens is found to be stationary which is identified with the Rayleigh regime. The onset of the Rayleigh mode in the different specimens is observed at larger $\Delta T_{c}$ for an increasing water content at constant molar concentration of AOT of $c_{M}=0.15$. At the same time, the amplitude of the Rayleigh mode decreases which reflects the differences of the Prandtl number for an increasing water content. 


\begin{tabular}{c|ccccc} 
no & $\Delta T_{c}^{p}[\mathrm{~K}]$ & $\kappa\left[\mathrm{m}^{2} / \mathrm{s}\right]$ & $\nu\left[\mathrm{m}^{2} / \mathrm{s}\right]$ & $L$ & $\sigma$ \\
1 & $0.890(5)$ & $8.91 \cdot 10^{-8}$ & $1.29 \cdot 10^{-6}$ & $1.68 \cdot 10^{-5}$ & 14 \\
2 & $0.951(7)$ & $8.52 \cdot 10^{-8}$ & $1.36 \cdot 10^{-6}$ & $1.19 \cdot 10^{-5}$ & 16 \\
3 & $1.339(7)$ & $7.04 \cdot 10^{-8}$ & $1.52 \cdot 10^{-6}$ & $0.54 \cdot 10^{-5}$ & 22
\end{tabular}

Table 4.2: Thermodynamic parameter estimations based on the convective amplitude in the Soret regime for the three different WDA specimen.

Decreasing $\Delta T$, the two-dimensional roll mode becomes unstable to stationary cross-rolls analogous to the transition to the Soret regime in the molecular binary mixture with $\psi>0$. None of the mixtures exhibits a large-scale structure $(k=0)$ and the velocity fields are found to loose structure to random velocity fluctuations with maximum amplitudes of approximately $1 \mu \mathrm{m} / \mathrm{s}$ at the lowest measured $r$. The primary consequence of the stationarity of the convective modes and the cross-roll state below the Rayleigh regime is that the microemulsion specimens are identified with a positive Soret coupling.

Assigning the results of Sec. 4.2.1 to the microemulsion system, the slope of the wave number independent velocity component $u$ versus $r=R / R_{c}=\Delta T / \Delta T_{c}^{p}$ allows for the estimation of $\kappa$. Therefore $u^{2}(r)$ is approximated by a linear fit yielding an amplitude in units of $[\mu \mathrm{m} / \mathrm{s}]$ which is compared with the theoretical value of pure fluid convection in units of $\kappa / d$ 94. Table 4.2 summarises the estimations of $\kappa$ and the deduced values of the Prandt 2 and Lewis number. The ratio of the thermal diffusivities of specimen 1 and pure $n$-decane is found to be consistent to within $10 \%$ with measurements of a microemulsion consisting of water, $n$-heptane and AOT [40] which further justifies the procedure.

In the water-less system (specimen 1) the amplitude closely resembles the characteristic of a molecular binary mixture (see Fig. 4.19). The transition to the Soret regime is particularly steep, slightly below the pure fluid threshold $(r=0.98(2))$. As for the case of the molecular mixture, the wave number $k=2.7(1)$ of the Rayleigh mode decreases in approaching $r=1$ to $k=2.5(1)$. The inset of Fig. 4.19 depicts the velocities in the Soret regime. It is noticeable that both components exhibit considerable scatter which is primarily resulting from the three-dimensionality of the structures. Secondly, as pointed out above, the convective state is not relaxed in this regime. However, $u$ visibly follows a linear trend comparable to the binary mixture case pointing to $r_{c}$

\footnotetext{
${ }^{2}$ The dynamic viscosity of the dehydrated micells (specimen 1) can be calculated using Einstein's formula, $\mu_{s}=1+2.4 \eta+14.1 \eta^{2}$ for non-interacting spheres 73 . With a view to specimen $2, \nu$ is estimated based on experimental data on an water/n-heptane/AOT system [40] and the value for the critical composition of 3 can be found in literature [11.
} 


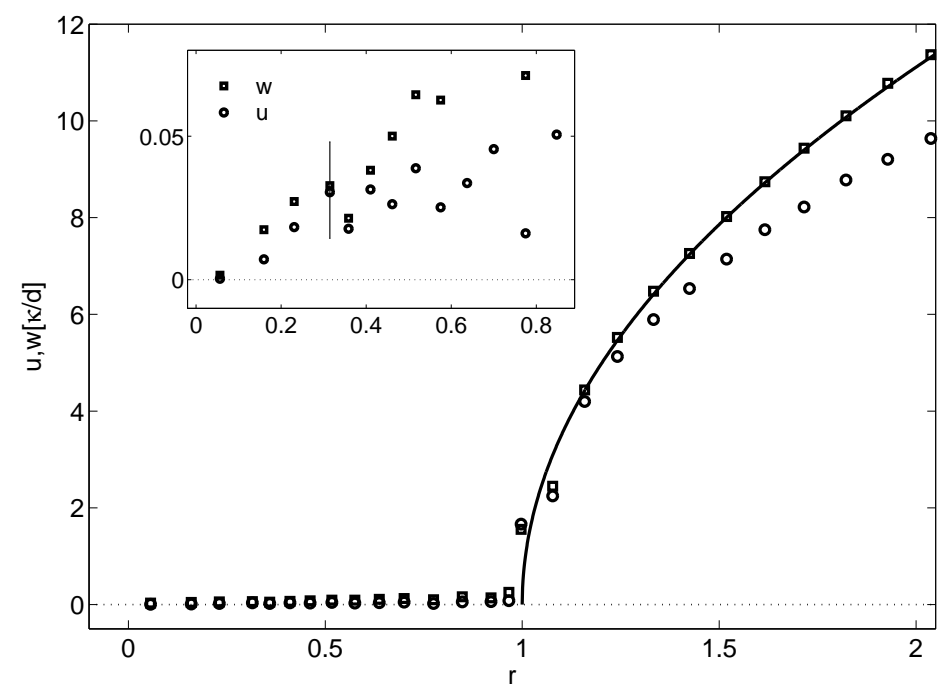

Figure 4.19: $u$ and $w$ versus $r$ measured in the water-less micellar solution (specimen 1); the solid line denotes the pure fluid amplitude.

very close to $r=0$. In comparison to 1 , the transition steepens in the case of specimen 2 leading to very small amplitudes of the Soret mode, cp. Fig. 4.20. Moreover, the amplitude is found to vanish rapidly in the measurement noise background (of approximately $0.018 \kappa / d$ ) accompanied by the dissolution of coherent structures at $r=0.85(2)$.

The transition from the Rayleigh mode to the Soret regime is particularly sharp for a weak influence of concentration effects, i.e. small $L \psi$ [53]. The amplitude in the Soret regime is $L / \psi$-times smaller than that of the Rayleigh mode and a substantial deviation from the pure fluid amplitude in the transition regime requires a large contribution of concentration effects to the buoyancy, i.e. a large separation ratio or fast diffusion corresponding to a large Lewis number. With a view to specimen 1 and 2 the estimation of the Lewis number is two orders of magnitude smaller than typical values for molecular mixture. The concentration boundary layer is extremely thin and roll mode is not sustained or stabilised below $r \simeq 1$ as in the case for the binary mixture. Furthermore, as the volume fraction $\eta$ is very small for both mixtures, the colloid suspension analogy suggests $\psi$ to be small, too [32]. The difference of specimen 1 and 2 have to be attributed to the decrease of $L$ associated with the larger micellar diameter which is not compensated by a difference in $\psi$ although the larger meso-structure size and the slight increase in $\eta$ would also suggest a rise of $\psi$.

The vanishing of convective motion without the transition to a large-scale 


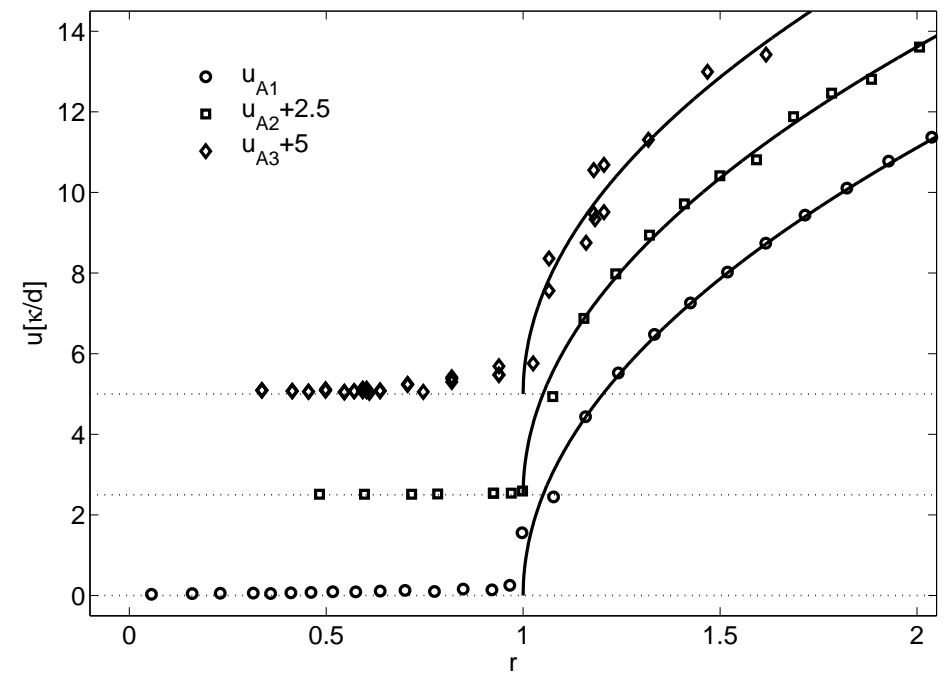

Figure 4.20: The maximum of the $u$-component representing the convection amplitude versus $r$ of the three different WDA specimens in comparison to the theoretical pure fluid solution.

structure is also a direct consequence of the Lewis number value $L$. The threedimensional flow structures of the Soret regime (which will be referred to as cross-rolls in the most general sense) are transfered into a single large-scale structure if concentration diffusion becomes comparable to advection. With a view to the microemulsion specimen the transition would require velocities of the order $\mathrm{O}\left(10^{-3}\right) \mu \mathrm{m} / \mathrm{s}$ and additional relaxation of the concentration boundary of the order of weeks. Both is experimentally unattainable. It should be remarked that the very onset of Soret convection in WDA in either case must be regarded beyond experimental prospects because the critical Rayleigh number is practically zero. Assuming $k_{c}=0$, the critical value is according to $R_{c}=720 L / \psi$ of the order $\mathrm{O}\left(10^{-2}\right)$ for $\psi=\mathrm{O}(10)$. The latter denotes the upper limit for $\psi$ available in fluid mixtures, found only for ferro-fluids, i.e. dispersions of metallic nano-particles.

The investigation of the critical mixture (specimen 3) was significantly hampered by experimental difficulties. The mixture exhibited non-negligible turbidity after injection into the cell which led to a reduced observability of the tracer particles. Therefore, the evaluation process had to be extended by an intensity average 3 prior to ensemble correlation evaluation. However, the inferior image density could not be fully compensated and the resulting amplitudes are rather noisy. Nevertheless, the slope of $u$ in Fig. 4.20 clearly demonstrates the relatively large amplitudes of the cross-roll state below the Rayleigh mode

\footnotetext{
${ }^{3}$ Intensity averaging in $\mu \mathrm{PIV}$ evaluation means that the tracer density in the image is artificially increase by the summation of a series of images.
} 
of specimen 3. In other words, the transition to the Soret mode is stretched with respect to 1 and 2. Taking into account the comparatively larger volume fraction $\eta=0.073$ and the large hydrodynamic radius of the micellar aggregates, the decrease of $L$ is obviously over-compensated by an increase of the separation ratio $\psi$. This observation suggests the separation ratio to be increased for larger aggregates which can be associated with stronger attractive interactions for larger contact areas of the micelles. As in the case of specimen 2 the amplitude signal is found to vanish in the measurement noise at approximately $r=0.49(3)$ in agreement with the above given argumentation.

In consistency with the argumentation in Sec. 2.3 the WDA specimens do exhibit a positive Soret coupling $\psi>0$ clearly demonstrated by stationary cross-roll structures below $r=1$. Accordingly, the Soret regime behaviour can be understood in analogy to colloidal suspensions with comparable Lewis numbers and separation ratios. A crude estimation of the separation ratio of the critical composition by comparison with colloid suspensions yields $\psi=$ $\mathrm{O}(1)$. The critical mixture, specimen 3, obviously exhibits a very pronounced Soret effect.

\subsubsection{Effects of Impurities}

The most pronounced effect of the critical mixtures' instability is a noticeable increase of turbidity as mentioned earlier. At the same instant, the criticality of the WDA specimen is noticeably altered after filling the convection cell. The phase separation for example can be found to set in inhomogeneously, i.e. not the whole layer undergoes transition at same temperature, pointing at considerable composition differences and local impurities. As a result of the extreme relaxation time of diffusion homogenisation, impurities are "frozen in". It is noted that the loss of criticality was observed also in WDA specimen not containing tracers and that several specimen were subjected to considerable aging albeit the fluid was thoroughly sealed.

A possible source of impurity is the cleaning procedure of the cell and the PTFE tubes connecting the outer reservoirs. An adaption by means of waterless cleansing and extended drying remained without appreciable improvement. The second and more important possible explanation for the instability of the microemulsion are the free surfaces in the reservoirs. It is known that trapped air might alter the criticality of mixtures due to contamination by moisture. Furthermore the free surface might lead to local concentration differences. The reservoirs are necessary since a partition of the tracer particles required for $\mu \mathrm{PIV}$ typically segregate, stick to surfaces, or agglomerate in regions of low shear. Therefore, the ability to mobilise particles is indispensable with a view to $\mu \mathrm{PIV}$ measurements.

In partial conclusion, the difficulties of the preservation of criticality of WDA are understood to result from the fluid storage and handling as the influence of tracers has been shown not to have substantial impact. However, these 
difficulties have not been resolved and led to abandoning further investigations of convection in the critical mixture in approaching the phase transition temperature. 


\subsection{Convective Pattern Formation in a Microemulsion}

In this section the pattern evolution of convection in a microemulsion of critical composition will be presented and discussed based on shadowgraph visualisation. The experiments cover a temperature range from a state far apart the critical region to the immediate vicinity of the critical point.

The experiments refer to a cylindrical Rayleigh-Bénard cell of $d=1.8 \mathrm{~mm}$ in

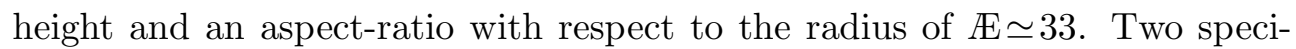
mens of the critical composition of WDA (specimen 3 of Sec. 4.3.1, $\omega=40.8$, $\eta=0.073$ ) were used and the near-criticality was verified directly after filling the cell. The experimental path, in this investigation is reverse with respect to the preceding sections, as $\Delta T$ is increased at a fixed top plate temperature $T_{t}$. Thus the distance to the critical consolute point $\epsilon=\left(\bar{T}-T_{c}\right) / T_{c}$ is decreased for consecutive steps. The system was allowed to relax approximately $2000 \mathrm{~s}$ prior to image acquisition. If phase separation occurred during the measurement, the fluid was cooled down to $T_{b} \ll T_{c}$ at large $\Delta T$ to invoke convective mixing. The re-homogenisation of the microemulsion was directly observable as the fluid turbidity went through a maximum and cleared off rapidly.

Due to the relatively weak image signal of the convective structures in WDA, especially at onset, the shadowgraph was aligned close to meeting the first caustic, i.e. maximum sensitivity. The shadowgraph images have been normalised in order to minimise background effects according to

$$
\tilde{I}(x, y)=\frac{I(x, y)-\langle I(x, y)\rangle}{\langle I(x, y)\rangle}, \quad\langle I(x, y)\rangle=\frac{1}{n} \sum_{i=1}^{n} I\left(x, y, t_{i}\right)
$$

where $I$ denotes the intensity, brackets indicate pixel-wise averaging, and $n$ is the number of images of the series. Furthermore, the grey-level distribution of the images have been inverted and re-adjusted to enhance the visibility of the pattern.

In addition to the shadowgraph visualisation, the heat transfer was recorded in terms of the power input to the bottom plate heater which is proportional to the Nusselt number $N$. The thermodynamic parameters to calculate Nusselt number are not a priori known for WDA. However, the Soret regime is not observable in the data. Nevertheless, the onset of the Rayleigh mode is indicated at $\Delta T_{c}^{p}=5.38(1) \mathrm{K}$ which is not noticeably shifted for the different mean temperatures measured. This value is used to scale the temperature difference $r=\Delta T / \Delta T_{c}^{p}$.

\subsubsection{Off-Critical Convection in WDA}

Far apart from the phase separation temperature, the thermodynamic parameters of WDA can be anticipated to be determined by the composition only and their variation with simultaneously increasing Rayleigh number and mean temperature of the layer $\bar{T}$ is expected to be negligible. The experiments with 

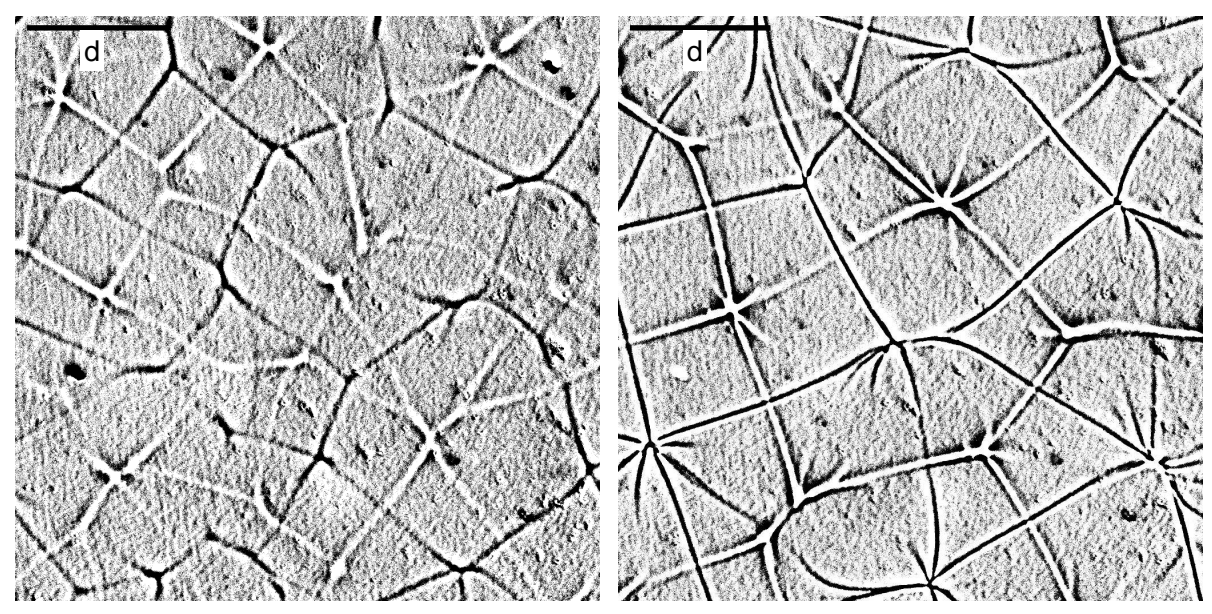

Figure 4.21: The first observable, spoke-like structures in the Soret regime of WDA $r=0.52(1)$ (left) and the evolving square-like pattern at $r=0.89(1)$ (right) in the off-critical region.

the top plate temperature held approximately $15 \mathrm{~K}$ below the critical temperature $T_{c}$, which implies the bottom temperature $T_{b}$ to be several $\mathrm{K}$ below $T_{c}$, are regarded as off-critical.

It should be noted that a large-scale structure cannot be observed using shadowgraph visualisation. However, according to the argumentation of the preceding section it is assumed that such a structure does not develop on the experimental time scale.

The first detectable structure is a stationary spoke-like pattern at approximately $r=0.52(1)$ depicted on the left hand side of Fig. 4.21 This pattern develops into increasingly ordered, square-like structures for further increasing $r$. The term "square-like" refers to the comparative pattern in Soret convection of molecular binary mixtures and does not apply rigorously since several defects, especially of triangular shape, are visible. This observation might be accidental although an increasing influence of thermal effects suggest the system to converge to the molecular binary fluid behaviour. These structures are subjected to limited meandering motion, i.e. they are regarded as quasistationary.

The induction time of convection in the Soret regime is considerably shorter than expected based on the diffusion relaxation of the system. Thus, it is understood that the concept of the separation of time scales applies for the WDA system in agreement with the separation ratio estimation of the preceding section.

The transition to Rayleigh mode at $r \simeq 1$ is initially visible in a progression of the meandering motion, i.e. a pattern re-arrangement, which reflects the competition of different roll modes (Fig. 4.23). The cylindrical geometry of 


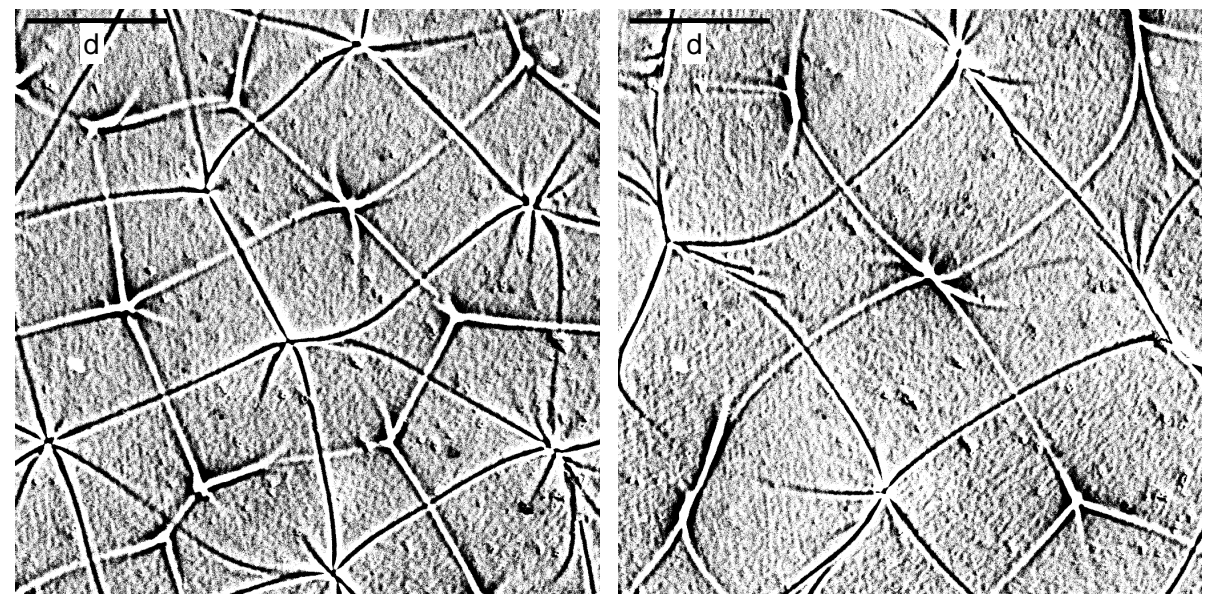

Figure 4.22: Square-like structures in the late stage of the Soret regime $r=0.92(1)$ (left) and $r=0.95(1)$ (right).

the convection cell favours spatial re-orientation in this regime, since none of the roll modes is selected by the lateral boundaries. Therefore, the transition regime gives rise to meander structures dominated by parallel rolls which persist to $r>1$. Remarkably, the size evolution of the pattern with increasing Rayleigh number is reverse to the case of molecular binary mixtures, i.e. the wave number decreases with increasing Rayleigh number.

Returning to the initially observed pattern, similar spoke structures have been reported for molecular binary mixtures and colloid solutions with negative separation ratio $\psi<0$ heated from above $r<0$ [71, 19]. In this case thermal expansion promotes a stable stratification while convection is solely induced by concentration fluxes. In the experiments of PORTA et al. [71 a sudden quench of the system to $r=-2$ has been reported to lead to initially small scale convective structures $\left(k \simeq k_{c}^{p}\right)$ appearing approximately one vertical diffusion relaxation time $\tau_{v, D}=d^{2} / D$ after the quench $\left(L=\mathrm{O}\left(10^{-2}\right), \psi=-0.24\right)$. The structures have been found to coarsen in time reaching a spoke-like state after $4 \tau_{v, D}$ at a wave number of $k \simeq k_{c}^{p} / 2$ which indicates convection to be initiated on small scales, comparable to salt-fingering. Salt-fingers consist of high-concentration and higher temperature fluid which sinks into colder fluid layers due to the large difference of thermal and concentration diffusivity. The heat exchange with the ambient fluid is much faster than concentration diffusion, thus the larger density of the high concentration fluid is preserved during sinking and cooling which drives the dynamics.

The pattern evolution of the spoke structures and especially the wave number decrease is attributed to nonlinear coupling of the convective modes. In close relation, the convection patterns in colloid suspensions with $\psi=\mathrm{O}(-1)$ and $L=\mathrm{O}\left(10^{-4}\right)$ have been observed to be initially disordered developing into 


\subsection{Convective Pattern Formation in a Microemulsion}

a spoke state very swiftly $\left(t \ll \tau_{v, D}\right)$ and saturating in the spoke state thereafter [19].

The spoke pattern observed in the present experiment can be understood in close analogy to the colloid suspension experiment cited above. The small Lewis number of the microemulsion in combination with an apparently large separation ratio leads to a very thin but highly unstable concentration boundary layer. At the same time the temperature gradient in the fluid layer becomes a nearly irrelevant variable to the problem since the heat diffusion is much faster than concentration diffusion leading to an homogeneous temperature gradient even in presence of convection. Therefore, the present experiment is directly comparable to heating from above at a negative separation ratio. The onset of convection is fluctuation induced, i.e. initiated by concentration plumes rising from the lower boundary layer, which gives rise to the development of small-scale roll structures leading to the spoke pattern. The velocity induced by the plumes rapidly alters the local concentration distribution thereby initiating larger scale flow. The initiation is spatially inhomogeneous over the layer, i.e. many differently oriented roll modes are generated. The pattern evolution, i.e. the decreasing wave number with increasing $r$ is mainly attributed to subsequent mode coupling corresponding to coarsening. Due to the extreme diffusion relaxation time of the WDA microemulsion, the initial spoke state is slowly coarsening which results in a small change of the wave number (cp. Fig. 4.22).

Strikingly, spoke-like pattern corresponding to Fig. 4.21] are well-known to occur in pure, large Prandtl number fluids at Rayleigh numbers of the order $R=\mathrm{O}\left(10^{6}\right)$ [116] beyond the instability of the bimodal regime in the transition regime to turbulent convection. The analogy between pure fluid convection and concentration driven convection at largely different Rayleigh numbers can be understood by neglecting the thermal gradient for Soret convection at small Lewis numbers. Doing so, both situations are described by the same equations of the Boussinesq approximation incorporating either the thermal or the solutal expansion. The control parameter for concentration driven convection, the solutal Rayleigh number, reads as

$$
\tilde{R}=\frac{\beta g \Delta T d^{3}}{D \nu}=\frac{\psi}{L} R
$$

Given $\psi / L=\mathrm{O}\left(10^{-4}\right)$ and typical Rayleigh numbers of the Soret regime of $R=\mathrm{O}\left(10^{2}\right)$ the similarity of the two convection states is obvious.

Noticeably, both of the pattern in WDA exhibit additional branches at the knots of the structure which, for increasing $r$, are found to drift or "blink", i.e. appearing and vanishing after finite times. These branches lead to a modulation of the basic state which constitutes a slow shivering motion of the knots. This effect will be treated in greater detail below as the modulation gains importance in approaching the phase transition temperature $(\epsilon \rightarrow 0)$. However, the presence of the branches, superimposing the basal pattern, indicates high 


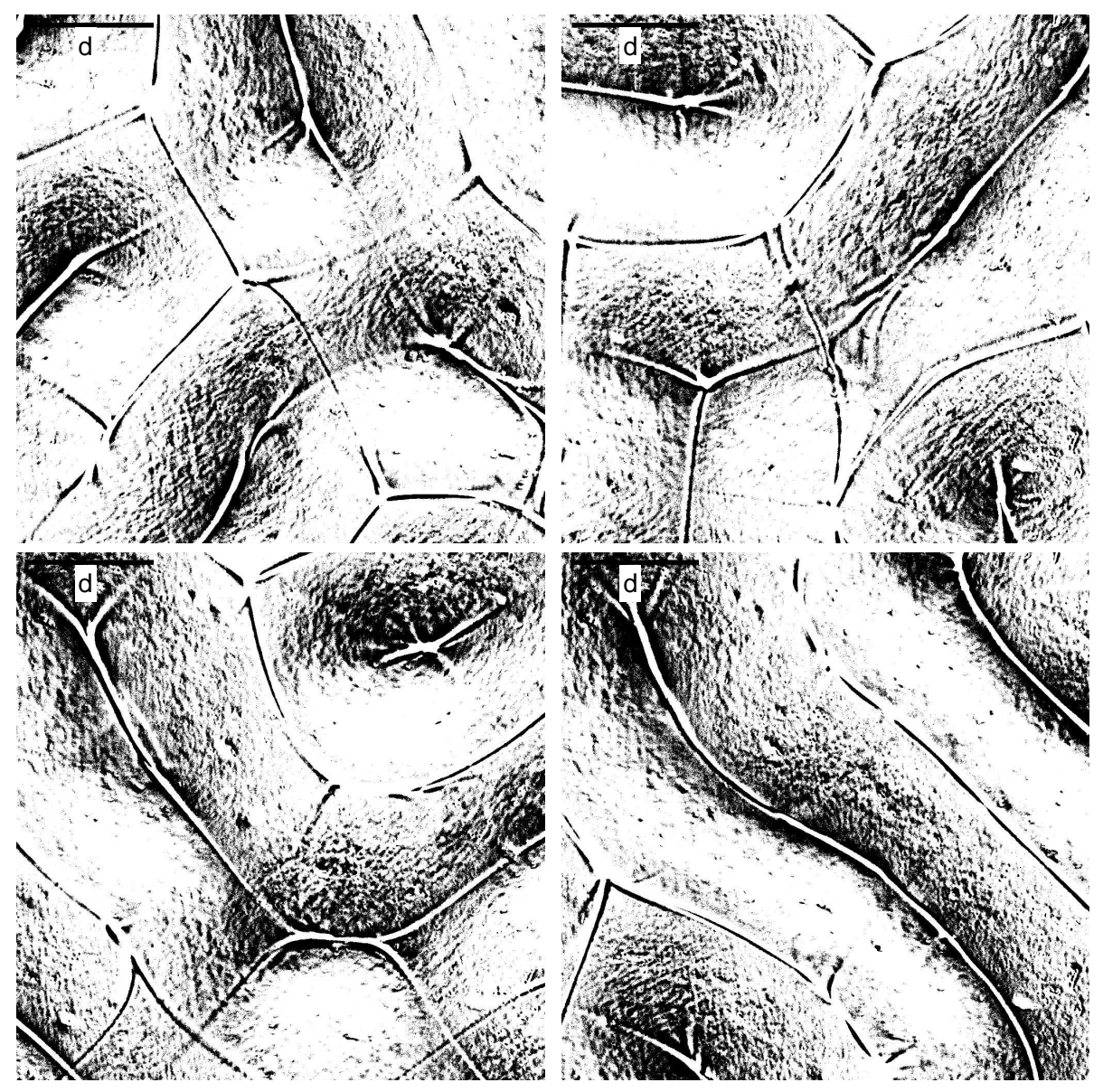

Figure 4.23: An example of competing square and roll structures in the transition to the Rayleigh regime $r=1.02(1)$.

instability and large local stresses which are identified with the concentration boundary layers. The branches are understood to result from the entrainment of lower concentration fluid which invokes a local destabilisation of the flow, forming a branch, due to the large value of $\psi$.

The convective pattern observed in WDA apart from the critical region conform the morphology of purely Soret driven convection in colloid suspensions with large separation ratios. Close to the transition to the Rayleigh regime, the succession of ordered, square-like, cross-rolls prior to a regime of competing cross-roll modes reflects the transition to thermally driven convection. However, at lower $r$ the spoke pattern together with finite branching indicates a local driving mechanism which is identified with the concentration boundary layer. An important argument to support the binary fluid analogy is that the small Lewis number of the critical composition effectively rules out structural 


\subsection{Convective Pattern Formation in a Microemulsion}

relaxation. Using the estimations given in Sec. 4.3.1, the vertical diffusion relaxation in the cylindrical cell is approximately $\tau_{v, D}=17 \mathrm{~h}$ and $\tau_{h, D}$ is of the order of weeks. Therefore, it follows that even if concentration driven convection has set in after a considerably shorter time, the structural development is not completed within the time-frame of the experiment.

\subsubsection{Convection in the Vicinity of Phase Transition}

In the first instance, approaching the critical phase transition temperature $\bar{T} \rightarrow T_{c}$ it is observed that the Rayleigh number at which an initial convection pattern becomes observable is reduced. At distances of $\bar{T}=T_{c}-15 \mathrm{~K}$ the very first structures are visible at approximately $r=0.5$ whereas at $T_{c}-1 \mathrm{~K}$ the initial pattern appear at $r \simeq 0.1$. The decrease of $r$ does not indicate a distinct relation to the distance to the critical point $(\epsilon)$ and repeated measurements at equal distance to $T_{c}$ exhibit considerable deviations of $r$ for different experimental runs.

An interesting pattern can be observed at the onset of the Soret mode approaching the critical phase transition temperature $T_{c}$. As depicted in Fig. 4.24, this pattern consists of initially ordered small-scaled roll structures with a waver number $k_{c}>k_{c}^{p}$. The initial ordered roll structure appears to be stationary albeit bound to a very narrow $r$ interval. This pattern is fairly unexpected especially since it was observed already at $T_{c}-5 \mathrm{~K}$. Increasing $r$, the small-scale rolls are observed to be transfered into small scaled disordered structures by a cascade of knot-bifurcations which drift along the basic pattern. Further increasing $r$, the knot density as well as the unsteadiness of the pattern grows leading to fully disordered structures (left side of the second row of Fig. 4.24). The latter represents the transfer to the spoke structures associated with the intermediate Soret regime (on the right of the second row of Fig. 4.24).

Increasing the mean temperature $\bar{T}$ leads to an earlier onset of the smallscale structures and a more rapid dissolution of those. In the ultimate vicinity of $T_{c}$ the aforementioned transfer of the small-scale pattern to fully disordered structures is observed to directly lead to incipient spinodal decomposition. This is directly visible in the form of small droplets of sharply imaged by the shadowgraph. The effect of phase separation will be subject of scrutiny below (Sec. 4.4.3).

From the dissolution of the small-scale pattern for large enough $r$ and distances to the critical temperature and their subsequent transition to spoke-like larger-scale structures, it is obvious that the pattern has to be attributed to a strong concentration stratification of the microemulsion. The disordered structures of Fig. 4.24 resemble the morphology of transient relaxation oscillations in two-layer convection. These oscillations are understood as capillary waves of a fluid-fluid interface driven by surface-tension effects, i.e. the Marangoni instability [119]. Especially, the late stage morphology of the small-scale struc- 


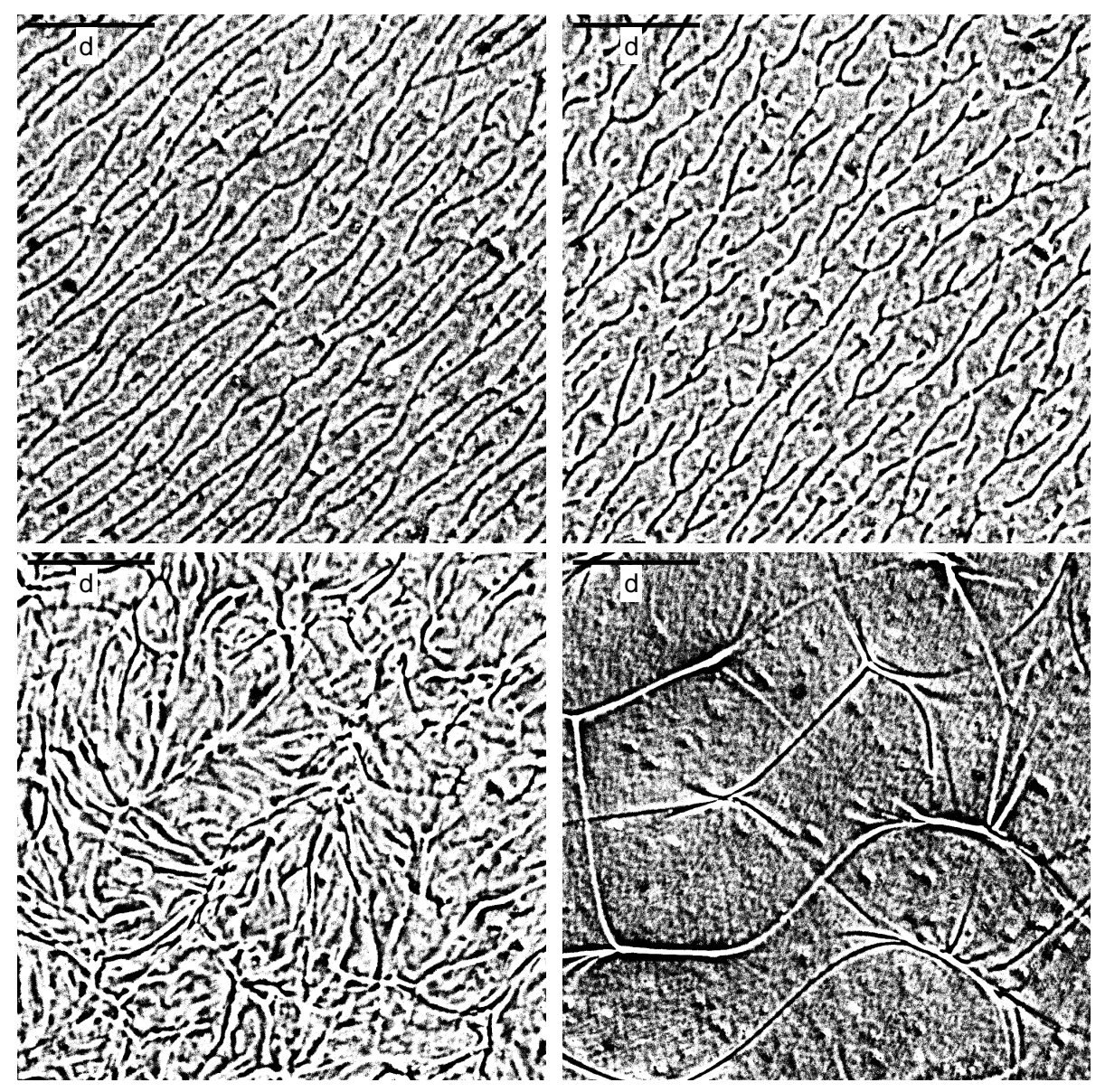

Figure 4.24: Small-scale structures as the initial pattern of the Soret mode and its structural development via knot-bifurcations, disordered small-scale structures and large-scale spoke pattern with increasing $r=0.32(1)$ to $r=0.71(1)$ (from left to right and top to bottom).

tures is equivalent to interfacial turbulence. For miscible phases, interfacial turbulence imposes mixing, leading to the dissolution of the stratification. However, the oscillation regime in two-phase Marangoni convection requires a "sharp" interface.

Combining the observations, the small-scale structures in the present experiment result from phase separation which in the first instance is unexpected at temperatures still of the order of $\mathrm{O}(1 \mathrm{~K})$ below $T_{c}$. On the other hand it has been stressed already that the concentration gradient is particularly steep due to the extremely slow concentration diffusion. Directly at the lower boundary, the concentration gradient attains its stationary value, $\nabla c=k_{T} / T \nabla T$, 


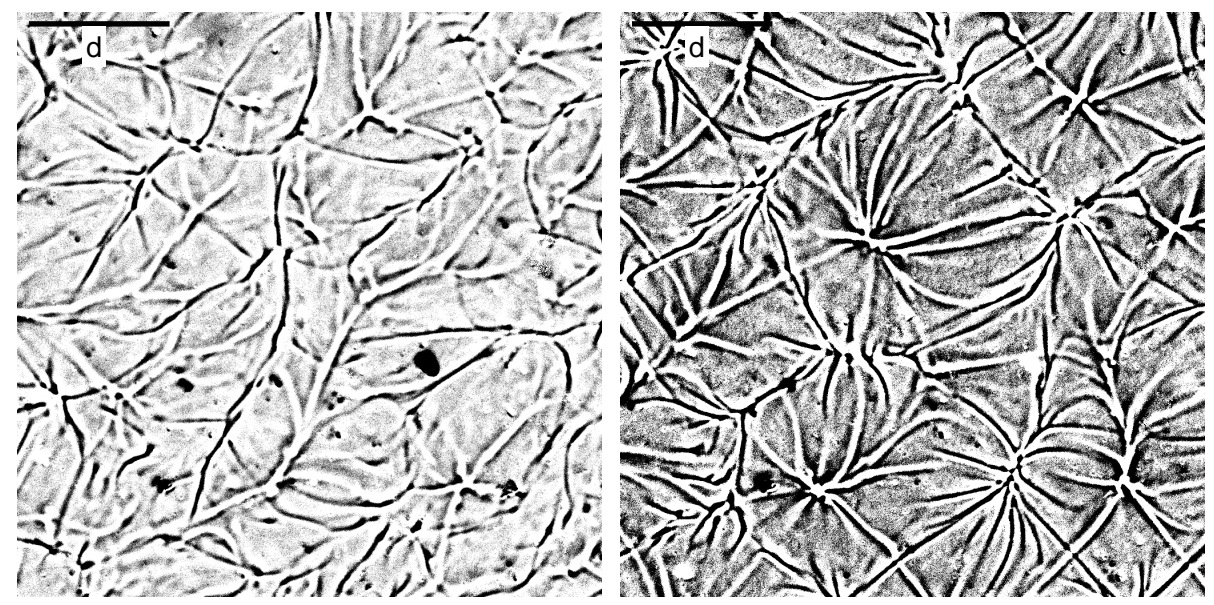

Figure 4.25: Disordered convective pattern in the Soret regime approaching the phase transition $r=0.32$ (1) (left) and $r=0.74$ (1) (right).

rapidly due to the impermeability condition. A concentration boundary layer of thickness $\delta_{c}$ is formed which growth as $\delta_{c} \sim \sqrt{D t}$, i.e. remaining of the order of $\mathrm{O}(10 \mu \mathrm{m})$ for subsequent experimental steps. Therefore, the concentration stratification can excesses the critical concentration threshold leading to localised phase separation, a phenomenon which has been also noticed in experiments on polymer solutions 70 4. The separation in the stratified fluid is non-critical and restricted to very thin layers. Hence, there is no noticeable blurring of the fluid visible in the visualisation.

The sub-layer of separated higher concentration microemulsion becomes unstable as a function of layer thickness and thermal driving leading to Marangoni convection. It should be noted that this state remains confined because both the species diffusion coefficients and the phases' viscosities stabilise the system against larger scale motion

$$
\frac{D^{(+)}}{D^{(-)}}>1 \quad \text { and } \quad \frac{\nu^{(+)}}{\nu^{(-)}}>1
$$

where the superscripts $(+)$ and $(-)$ denote dense and dilute microemulsion phase respectively.

This effect aggravates approaching the phase transition temperature. The closer the system is to $T_{c}$, the smaller the Lewis number and, hence, the earlier the onset of phase separation. Remarkably, numerical simulation of a binary fluid at one thermal boundary exceeding the critical temperature have been reported to exhibit ordered small-scale roll structures [101]. These formation

\footnotetext{
${ }^{4} \mathrm{~A}$ sharp interface could result from non-adequate re-mixing of a completely phase separated state of a preceding run. This cannot be ruled out in general. However, the small scale structures were also observed in freshly prepared WDA specimen.
} 


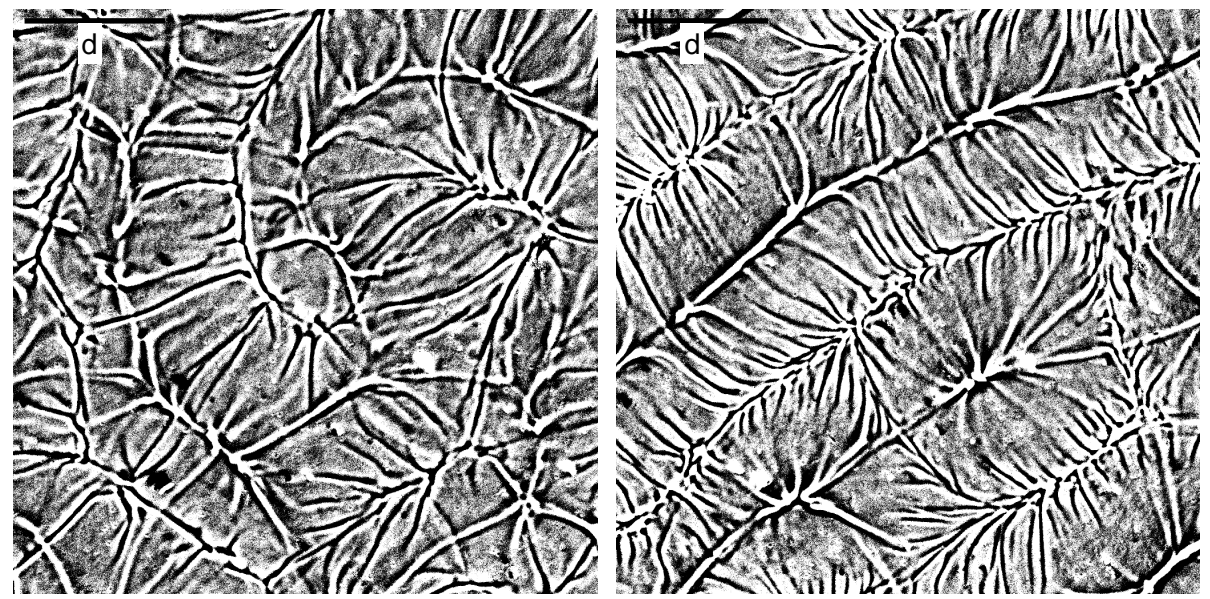

Figure 4.26: Superposition of the roll pattern at $r=1.03(1)$ by additional branches close to $T_{c}$.

of such confined convective structures is also attributed to the Marangoni instability and a balancing effect of large viscosity of the separated fluid further sustaining the argumentation given above.

As a consequence of the phase separation in the boundary layer, the critical region cannot be approached preserving the homogeneity of the mixture in absence of convection. Therefore, critical effects could only be studied if the concentration distribution is largely homogenised, i.e. in the Rayleigh regime. It should be noted that the same argumentation holds for molecular binary mixtures close to the consolute point due to the strong divergence of the concentration diffusion.

In the intermediate Soret regime, the spoke patterns are found to exhibit an increasing number branches as the critical point is approached (cp. Fig. 4.26). As an effect of the branches drifting and their time-dependent nature, the pattern exhibit permanent random re-arrangement below the transition regime to the Rayleigh mode. The time-dependence of the branches should be thought of as new branches emerging, most often at a knot, drifting along the roll and disappearing randomly. Noticeably, in absence of a knot, the branches orient themselves at approximately $90^{\circ}$ with respect to the roll pattern.

According to the description in the preceding section, the branches are identified with low concentration fluid originating from the boundary layer or the separatrix which is entrained by the mean flow. Due to the large buoyancy forces resulting from the large separation ratio, the concentration boundary layer becomes particularly unstable. As $\psi$ increases towards $T_{c}$ and even diverges in the critical region, the instability of the boundary layer grows $(\epsilon \rightarrow 0)$. The closer the system is to the critical temperature, $T_{c}$, the larger the probability of plumes to emerge from the boundary layers and, therefore, the larger 

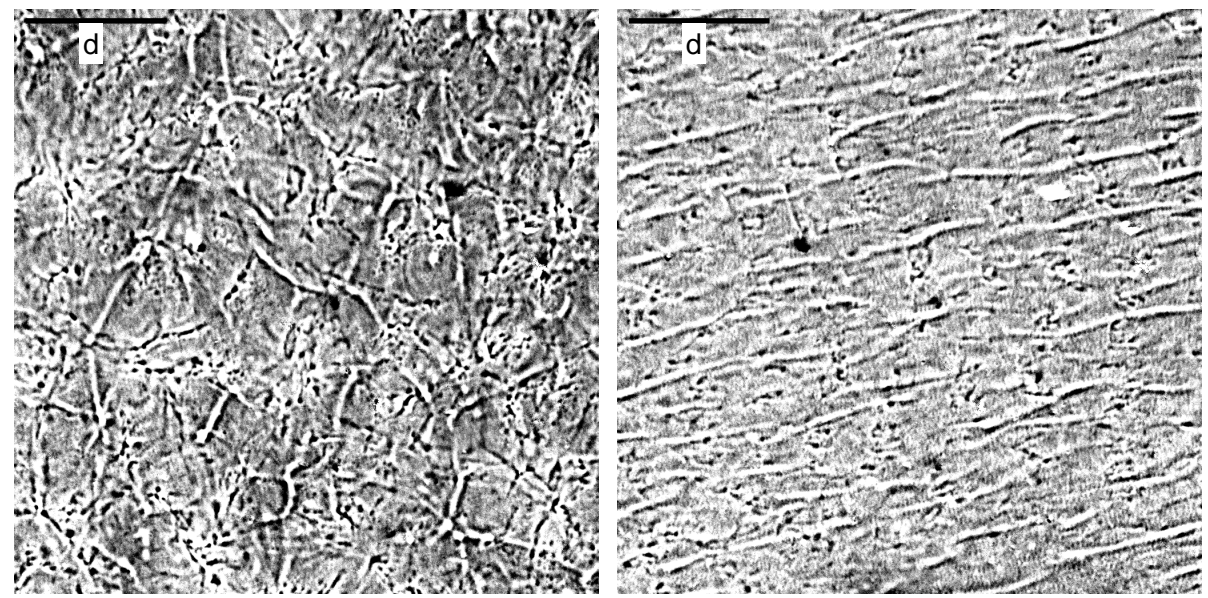

Figure 4.27: Plumes of separated concentration droplets locally rising from the lower boundary layer superimposing the polygonal pattern at $\bar{T} \simeq T_{c}$ and $r=0.83(1)$ (left) and TW structures of aligned droplet streaks close to the onset of spinodal decomposition at $r=0.89(1)$ (right).

the number of additional branches. The convection amplitude is also observed to noticeably increase in approaching $T_{c}$ due to the increasing separation ratio. Thus, the branching is a direct result of the growth of the separation ratio and represents the main impact of approaching $T_{c}$ in presence of a developed convection state. It is interesting to note that the convection pattern is not observed to become completely unstructured or disordered prior to discernible decomposition. In conclusion there is no evidence of compressibility effects in the vicinity of the critical temperature.

Another peculiar effect of approaching $T_{c}$ is that the transition to the Rayleigh mode is increasingly stretched to larger Rayleigh numbers $(r>1)$. The visualisation demonstrates the competition regime of roll and square structures to broaden. This can be definitely identified with the growth of the separation ratio. In agreement with theoretical predictions, the rise of $\psi$ amplifies the Soret effect while the Lewis number has a minor effect in the transition regime to the Rayleigh mode [53. Furthermore, the heat transfer of the Rayleigh mode loses steepness near the critical point. This can be understood based on the alteration of the fluid parameters, e.g. the Prandtl number in this region.

\subsubsection{The Effect of Incipient Decomposition}

Incipient spinodal decomposition becomes firstly visible in the form of small non-dissipating droplets being transported by the mean flow of the basal pat- 

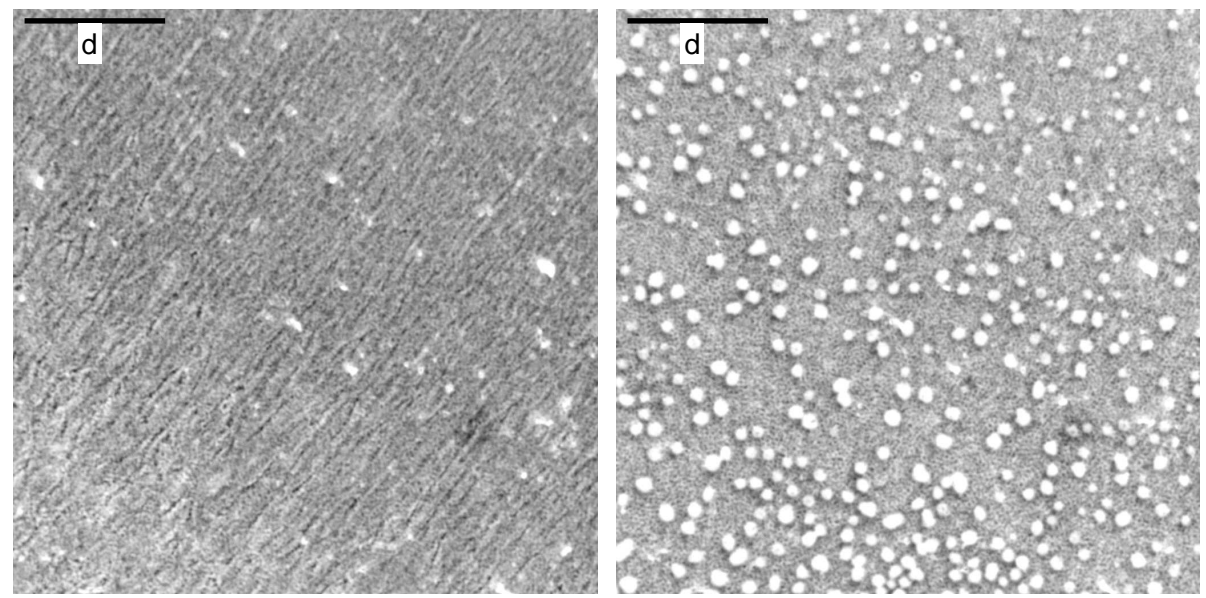

Figure 4.28: An early (left, $r=0.54(1)$ ) and a very late stage pattern of incipient spinodal decomposition (right, $r=0.79(1)$ ) at $\bar{T}>T_{c}$. The ripply substructure on the left image is due to a sub-layer of separated fluid.

tern. Figure 4.27 illustrates this state in limited quality only (the drops are the small black dots visible). The experiments demonstrate the decomposition to set in within the lower boundary layer while the temperature of the bulk fluid subjected to convective motion is still below $T_{c}$. As fluid from the boundary layer is injected into the mean flow by means of entrainment, high concentration beads are transported into the bulk. At this stage the concentrated phase is unable to follow the flow and disrupts the convective pattern leading to a state of arbitrary plume-like emergence. The system is transfered from a homogeneous fluid to an emulsion-like two-phase flow thereby invalidating the Boussinesq approximation. However, due to re-mixing the pattern is stationary and the droplet size is constant during the observation time.

It is interesting to note that at slightly larger $r$ the droplets align in parallel streaks moving as a single mode travelling wave while another aligned substructure travels at an angle of approximately $90^{\circ}$ below it. The streaks are hardly recognised in Fig. 4.27 because the droplets are still very small. The latter pattern can be associated with a sub-layer of separated liquid exhibiting capillary waves corresponding to the small-scale structure described above. This pseudo-TW has been observed crossing the phase separation at largely different $r$. It is concluded that this structure is inherent to phase separation subjected to a temperature gradient.

Phase separation itself is subjected to locally varying quasi-linear shear in presence of stationary convection in between adjacent rolls for example. Shear rates exceeding the thermal decay rate, $\tau_{\xi}^{-1}$ of thermal fluctuations, lead to an elongation of those and highly anisotropic spatial correlations [96. In the 
case of $S \tau_{\xi} \geq 1$ this will mainly result in a slightly delayed phase separation The alteration of $T_{c}$ has been quantified for binary mixtures subjected to uniform shear reading $\delta T_{c}=0.0832\left(16 \mu \xi_{0}^{3} S / k_{B} T\right)^{1 / 3 \nu}$ [96]. Estimating the shear rate for the stationary rolls of the Rayleigh regime assuming $k=k_{c}^{p}, d=3 \mathrm{~mm}$ and a velocity amplitude of $A=\mathrm{O}(0.1 \mathrm{~mm} / \mathrm{s})$ the shear rate is of the order $S=\mathrm{O}(0.1)$ which for $\xi_{0}=\mathrm{O}(10 \AA)$ yields $\delta T_{c}=\mathrm{O}(0.01 \mathrm{~K})$. The droplet structures are observed to occur at distinctly larger values of approximately $1 \mathrm{~K}$ above the initially measured $T_{c}$. In the light of the argumentation concerning localised phase separation the deviations are interpreted as an effect of the resulting inhomogeneity of the fluid specimens.

Further on, increasing $r$ from the state of pseudo-TW, all visible structures disappear except for small droplet-like pattern, the turbidity of the fluid increases remarkably, and macroscopic phase separation is indicated by large drops of one phase sampling out, cp. Fig. 4.28. The size of the separated macro-domains are obviously limited by convective motion which delays further coarsening in agreement with earlier findings [7. 


\section{Conclusions and Future Prospects}

\subsection{Conclusions}

Binary and pseudo-binary fluid convection has been investigated with special emphasise on the Soret regime of concentration dominated convective motion. An adaption of the $\mu \mathrm{PIV}$ technique developed in the framework of the present experiments has been successfully applied for the investigation of molecular binary fluid convection and convection in a pseudo-binary microemulsion.

The direct velocity amplitude measurements of purely Soret driven convection in the binary mixture confirm the existence of a single large-scale coherent flow structure filling the whole geometry of the convection cell in agreement with linear stability analysis. For the first time, this structure has been examined directly at onset and the critical Rayleigh number has been found to reasonably agree with the theoretical prediction. The analysis of the velocity data clearly attributes structural transitions of the convective flow field to the competition of diffusion and advection. Therefore, the stationary transitions from the large-scale structure to squares in the Soret regime and later to the roll state of the Rayleigh regime exhibit hysteresis due to the re-mixing imposed by advection.

The excursion on travelling wave convection at negative separation ratio have confirmed the velocity field to be comparable to the case of pure fluid convection in agreement with results of numerical simulations. Additionally, a confined travelling wave state has been considered at the front to the quiescent neighbouring fluid which revealed remarkable three-dimensionality of the flow due to the finite geometry of the convection cell.

According to the present measurements, the ternary microemulsion is regarded as a pseudo-binary mixture with a positive Soret effect in close analogy to colloid mixtures of comparable Lewis number. The experiments clearly show the dynamics of convection in microemulsions to be determined by the decoupling of thermal and concentration diffusivity. The very small concentration diffusion, i.e. the very small scale of the Lewis number, has been shown to lead to a number of peculiar effects in combination with an apparently large separation ratio. The system does not relax on experimental time scales and the dynamics are dominated by the concentration boundary layer resulting from the non-relaxed concentration gradient. The onset of Soret convection is spontaneously induced and the observed spoke pattern at onset indicates large stresses, i.e. a large separation ratio.

The main experimental interest of this work, the investigation of convection close to a critical consolute point, has led to the conclusion that due to the 
particularly steep concentration gradient in the closest proximity of heated side local phase separation is initiated well below the critical point. As a consequence, the homogeneity and criticality of the fluid is not preserved. This effect is not restricted to microemulsions since the concentration diffusion is strongly divergent in the critical region of consolute points of mixtures. Thus, critical effects on Rayleigh-Bénard convection could be studied in the Rayleigh regime only, where the concentration distribution is homogenised by advection. As it has been noted in the considerations about the transport properties of a mixture in the critical region, the divergence of the parameters not related to concentration is weak. Therefore, the observed Rayleigh mode is increasingly amplified by the increasing separation ratio. However, the roll structures of the Rayleigh regime do not indicate osmotic compressibility to be important.

\subsection{Future prospects}

In continuation of the convection experiments presented here, it would be most interesting to extend the direct velocity amplitude measurements to colloid mixtures in order to study the time evolution of the flow directly at the onset of convection possibly in connection with concentration distribution measurements. As the structure evolution is particularly slow, scanning techniques would allow for a resolution of volumes, capturing the whole characteristic. Additionally, localised or confined travelling wave structures can be promising to study in order to verify recent numerical simulation results.

A more challenging but still fascinating idea is to examine critical effects in the vicinity of the consolute point in an isothermal, planar Couette flow on micro-scales. Provided the critical region can be stabilised critical fluctuations could eventually grow to sizes comparable to the flow geometry which can be expected to lead to peculiar effects.

Proceeding on the investigation of critical effects in microemulsions, the extreme experimental difficulties with a view to the WDA microemulsion suggest to move to alternative, potentially more complex systems which are less susceptible to chemical impurities. 


\section{Bibliography}

[1] G. Ahlers. Effects of departures from the oberbeck-boussinesq approximation on the heat transport of horizontal convecting fluid layers. $J$. Fluid Mech., 98(1):137, 1979.

[2] S. Amokrane and M. Bouaskarne. Shape of the liquid vapor coexistence curve for temperature and density dependent effective interactions. Phys. Rev. E, 65:051501, 2002.

[3] M.A. Anisimov. Critical Phenomena in Liquids and Liquid Crystals. Gordon and Breach Science Publishers, 1991.

[4] M.A. Anisimov and S.B. Kiselev. Transport properties if critical dilute solutions. Int. J. Thermophys., 13(5):873, 1992.

[5] A. Arcoleo, M. Goffredi, and V. Turco Liveri. Electrical conductivity and permittivity of water-AOT-n-heptane microemulsions. J. Sol. Chem., 24(11):1135, 1995.

[6] K. Asami. Characterization of heterogeneous systems by dielectric spectroscopy. Prog. Polym. Sci., 27:1617, 2002.

[7] M. Assenheimer. Rayleigh-Bénard Convection in the Vicinity of the Thermodynamical Critical Point. PhD thesis, Weizmann Institute of Science, Israel, 1994.

[8] M. Assenheimer and Steinberg V. Rayleigh-Bénard convection near the gas-liquid critical point. Phys. Rev. Lett., 70(25):3888, 1993.

[9] W. Barten, M. Lücke, M. Kamps, and R. Schmitz. Convection in binary fluid mixtures. i. extended traveling waves and stationary states. Phys. Rev. E, 51(6):5636, 1995.

[10] R.J. Baxter. Percus-yevik equation for hard spheres with surface adhesion. J. Chem. Phys., 49(6):2770, 1968.

[11] R.F. Berg, M.R. Moldover, and J.S. Huang. Quantitative characterization of the viscosity of a microemulsion. J. Chem. Phys., 87(6):3687, 1987.

[12] P. Bergé and M. Dubois. Convective velocity field in the RayleighBénard instability: Experimental results. Phys. Rev. Lett., 32(19):1041, 1974. 
[13] M. Bestehorn. Numerical results of 3d convection in a binary mixture for a positive separation ratio. Physica D, 61:59, 1996.

[14] E. Bodenschatz, J.R. de Bruyn, G. Ahlers, and D.S. Cannel. Transition between patterns in thermal convection. Phys. Rev. Lett., 67:3078, 1991.

[15] M. Bouaskarne, S. Amokrane, and C. Regnaut. Hard core yukawa fluid with temperature and density dependent interaction: Phase diagram of the aot/water/decane microemulsion. J. Chem. Phys., 111(5):2151, 1999.

[16] F.H. Busse. The stability of finite amplitude cellular convection and its relation to an extremum principle. J. Fluid Mech., 30(4):625, 1967.

[17] A.M. Cazabat, D. Chatenay, and D. Langevin. Percolation and critical points in microemulsions. Faraday Discuss. Chem. Soc., 76:291, 1983.

[18] A.M. Cazabat, D. Langevin, J. Meunier, and A. Pouchelon. Critical behaviour in microemulsions. J. Physique - Lettres, 43:L-89, 1982.

[19] R. Cerbino, A. Vailati, and M. Giglio. Soret driven convection in a colloidal sulotion heated from above at very large solutal rayleigh numbers. Phys. Rev. E, 66:055301-1, 2002.

[20] S. Chandrasekhar. Hydrodynamic and Hydromagnetic Instability. Dover, 1981.

[21] S.H. Chen and J.S. Huang. Dynamic slowing-down and non-exponential decay of density correlation function in dense microemulsions. Phys. Rev. Lett., 55(18):1888, 1985.

[22] S.H. Chen, J. Rouch, F. Sciortino, and P. Tartaglia. Static and dynamic properties of water-in-oil microemulsions near the critical percolation points. J. Condens. Matter, 6:10855, 1994.

[23] T. Clune and E. Knobloch. Square pattern convection in binary fluids with experimental boundary conditions. Phys. Rev. A, 44(12):8084, 1991.

[24] M.C. Cross. Derivation of the amplitude equation at the RayleighBénard instability. Phys. Fluids, 23(9):1727, 1980.

[25] M.C. Cross. Travelling and standing waves in binary fluid convection in finite geometries. Phys. Rev. Lett., 57(23):2935, 1986.

[26] M.C. Cross and P.G. Hohenberg. Pattern formation outside of equilibrium. Rev. Mod. Phys., 65(3):851, 1993. 


\section{Bibliography}

[27] M.C. Cross and K. Kim. Existence of a codimension-2 point at the threshold of binary-fluid convection between rigid, impermeable boundaries. Phys. Rev. A, 38(1):529, 1988.

[28] M. D'Angelo, D. Fioretto, G. Onori, L. Palmieri, and A. Santucci. Study of the dynamics of water/aerosol OT $/ n$-heptane systems by dielectric relaxation measurements. Coll. Polym. Sci., 273:899, 1995.

[29] M. D'Angelo, D. Fioretto, G. Onori, L. Palmieri, and A. Santucci. Dynamics of water-containing sodium bis(2-ethylhexyl)sulfosuccinate (AOT) reverse micells: A high-frequency dielectric study. Phys. Rev. E, 54(1):993, 1996.

[30] K.S. Das and J.K. Bhattacharjee. Onset of convection in a binary mixture near a plait point. Phys. Rev. E, 61(5):5191, 2000.

[31] J.R. de Bruyn, E. Bodenschatz, S.W. Morris, S.P. Trainoff, Y. Hu, D.S. Canell, and G. Ahlers. Apparatus for the study of Rayleigh-Bénard convection in gases under pressure. Phys. Rev. E, 67(6):2043, 1996.

[32] J.K.G. Dhont. Thermodiffusion of interacting colloids. II. A microscopic approach. J. Chem. Phys., 120(3):1642, 2004.

[33] M. Dubois and P. Bergé. Experimental study of the velocity field in Rayleigh-Bénard convection. J. Fluid Mech., 85(4):641, 1977.

[34] H.-F. Eicke, R. Hilfiker, and M. Holz. Percolation phenomena in microemulsions of the one-component macrofluid type. Helv. Chim. Acta, 42(2):361, 1984.

[35] M.E. Fisher. The theory of equilibrium critical phenomena. Rev. Mod. Phys., 30:615, 1967.

[36] M.E. Fisher. Renormalization of critical exponents by hidden variables. Phys. Rev., 176:257, 1968.

[37] W.M. Gelbart and A. Ben-Shaul. The "new" science of complex fluids. J. Phys. Chem., 100:13169, 1996.

[38] M. Giglio and A. Vendramini. Thermal-diffusion measurements near the consolute critical point. Phys. Rev. Lett., 34(10):561, 1975.

[39] M. Gitterman. Hydrodynamics of fluids near a critical point. Rev. Mod. Phys., 50:85, 1978.

[40] F. Goffredi, V.T. Liveri, and G. Vssallo. Heat capacities and enthalpies of dilution of water/AOT/n-heptane microemulsions at $25^{\circ} \mathrm{C} . \mathrm{J}$. Coll. Interf. Sci., 151(2):396, 1991. 
[41] S.C. Greer, T.K. Bose, and J. Thoen. The density near a critical composition of the aot-water-decane microemulsion. J. Chem. Phys., 91(1):620, 1989.

[42] R:B. Griffiths and J.C. Wheeler. Critical points in multicomponent systems. Phys. Rev. A, 2(3):1047, 1970.

[43] D. Gutkowicz-Krusin, M.A. Collins, and J. Ross. Rayleigh-Bénard instability in nonreactive binary fluids. i. theory. Phys. Fluids, 22(8):1443, 1979.

[44] D. Gutkowicz-Krusin, M.A. Collins, and J. Ross. Rayleigh-Bénard instability in nonreactive binary fluids. ii. results. Phys. Fluids, 22(8):1451, 1979.

[45] R. Heinrichs, G. Ahlers, and D.S. Cannell. Traveling waves and spatial variation in the convection of a binary mixture. Phys. Rev. A, 35(6):2761, 1987.

[46] N. Hill, W.E. Vaughan, A.H. Price, and M. Davis. Dielctric properties and molecular behaviour. van Nostrand, London, 1969.

[47] T.P. Hoar and J.H. Schulman. Transparent water-in-oil dispersions: the oleopathic hydro-micell. Nature, 152:102, 1943.

[48] P.C. Hohenberg and B.I. Halperin. Theory of dynamic critical phenomena. Rev. Mod. Phys., 49(3):435, 1977.

[49] St. Hollinger and M. Lücke. Influence of the Dufour effect on convection in binary gas mixtures. Phys. Rev. E, 52(1):642, 1995.

[50] St. Hollinger, M. Lücke, and H.W. Müller. Model for convection in binary liquids. Phys. Rev. E, 57(4):4250, 1998.

[51] J.S. Huang and M.W. Kim. Critical behaviour of a microemulsion. Phys. Rev. Lett., 53(6):592, 1981.

[52] J.S. Huang, S.A. Safran, M.W. Kim, D.S. Grset, M. Kotlarchyk, and N. Quirke. Attractive interactions in micelles and microemulsions. Phys. Rev. Lett., 47(20):1462, 1984.

[53] B. Huke, H. Pleiner, and M. Lücke. Convection patterns in colloid solutions. Phys. Rev. E, 75:036203-1, 2007.

[54] D.R. Jenkins and M.R.E. Proctor. The transition from roll to square-cell solutions in Rayleigh-Bénard convection. J. Fluid Mech., 139:461, 1984.

[55] Ch. Jung and M. Lücke. Localized waves without the existence of extended waves: Oscillatory convection of binary mixtures with Soret effect. Phys. Rev. Lett., 89(5):054502-1, 2002. 


\section{Bibliography}

[56] Ch. Jung and M. Lücke. Travelling wave fronts and localized travelling wave convection in binary fluid mixtures. Phys. Rev. E, 72:026307-1, 2005.

[57] U. Kaatze and K. Giese. Dielectric relaxation spectroscopy of liquids: Frequency domain and time domain experimental methods. J. Phys. E: Sci. Instrum., 13:133, 1980.

[58] C. Kähler and U. Scholz. Transonic jet analysis using long-distance micro PIV. In 12th Int. Symp. on Flow Vis., September 10-14, German Aerospace Centre (DLR), Göttingen, Germany, 2006.

[59] P.K. Khabibullaev and A.A. Saidov. Phase Separation in Soft Matter Physics. Springer, 2003.

[60] M.W. Kim and J.S. Huang. Pressure induced critical phenomena of a microemulsion system. Phys. Rev. Lett., 54(1):46, 1985.

[61] E. Knobloch and D.R. Moore. Linear stability of experimental Soret convection. Phys. Rev. A, 37(3):860, 1988.

[62] P. Kolodner, Passner A., and R.W. Surko, C.M. Walden. Onset of oscillatory convection in a binary fluid mixture. Phys. Rev. Lett., 56(24):2621, 1986.

[63] P. Kolodner, H. Williams, and C. Moe. Optical measurements of the sort coefficient of ethanol/water solutions. J. Chem. Phys., 88(10):6514, 1988.

[64] W. Korneta, M.A. López-Quintela, and A. Fernández-Nóvoa. The convection patterns in microemulsions. In published at the International Center for Theoretical Physics, Miramare - Trieste, 1991.

[65] W. Korneta, M.A. López-Quintela, and A. Fernández-Nóvoa. The nonlinear evolution of spatio-temporal structures in microemulsions. Physica A, 185:116, 1992.

[66] M. Kotlarchyk, S.-H. Chen, and J.S. Huang. Temperature dependence of size and polydispersity in a three-component microemulsion by smallangle neutron scattering. J. Phys. Chem., 86(17):3273, 1982.

[67] M. Kotlarchyk, S.-H. Chen, and J.S. Huang. Critical behaviour of a microemulsion studied by small-angle neutron scattering. Phys. Rev. A, 28(4):508, 1983.

[68] M. Kotlarchyk, S.-H. Chen, J.S. Huang, and M.W. Kim. Structure of three-component microemulsions in the critical region determined by small-angle neutron scattering. Phys. Rev. A, 29(4):2054, 1984. 
[69] C.Y. Ku, S.H. Chen, J. Rouch, and P. Tartaglia. Structure and dynmics of water-in-oil microemulsions near the critical and percolation points. Int. J. Thermophys., 16(5):1119, 1995.

[70] J. Kumaki, T Hashimoto, and S. Garnik. Temperature gradients induced phase separation in a miscible polymer solution. Phys. Rev. Lett., 77(10):1990, 1996.

[71] A. La Porta and C.M. Surko. Convective instability in a fluid mixture heated from above. Phys. Rev. Lett., 80(17):3759, 1995.

[72] A. La Porta and C.M. Surko. Dynamics of two-dimensional travelingwave convection patterns. Phys. Rev. E, 53(6):5916, 1996.

[73] J.R. Lalanne, B. Pouligny, and E. Sein. Transport properties of dilute inverted micelles and microemulsions. J. Phys. Chem., 87:696, 1983.

[74] L.D. Landau and E.M. Lifshitz. Fluid Mechanics. Pergamon, 1959.

[75] M.J. Lawrence and G.D. Rees. Microemulsion-based media as novel drug delivery systems. Adv. in Drug Del. Rev., 45:89, 2000.

[76] T.D. Lee and C.N. Yang. Statistical theory of equations of state and phase transitions, ii. lattice gas and ising model. Phys. Rev., page 410, 1952.

[77] J. Lenglet, A. Bourdon, J.C. Bacri, and G. Demouchy. Thermodiffusion in magnetic colloids evidenced and studied by forced Rayleigh scattering expeiments. Phys. Rev. E, 65(3):031408, 2002.

[78] L. Letamendia, E. Pru-Lestret, P. Panizza, J. Rouch, F. Sciortino, P. Trataglia, C. Hashimoto, H. Ushiki, and D. Risso. Relaxation phenomena in AOT-water-decane critical and dense microemulsions. Physica A, 300:53, 2001.

[79] O. Lhost and J.K. Platten. Experimental study of the transition from nonlinear traveling waves to steady overturning convection in binary mixtures. Phys. Rev. A, 40(8):4552, 1989.

[80] M.A. López Quintela. Observation of spatial dissipative structures in microemulsions. J. Non-Equ. Thermodyn., 14:279, 1989.

[81] M.A. López-Quintela, A. Fernández-Nóvoa, and W. Korneta. Convection in microemulsions. In T. Riste and D. Sherrington, editors, Spontaneous formation of space-time structures and criticality. Kluwer Academic Publishers, 1991. 


\section{Bibliography}

[82] M.A. López-Quintela, C. Tojo, M.C. Blanco, L.G. Rio, and J.R. Leis. Microemulsion dynamics and reactions in microemulsions. Cur. Op. Coll. Interf. Sci., 9:264, 2004.

[83] F. Malamace, N. Micali, and S.H Chen. Spinodal decomposition in systems containing surfactant molecules. Phys. Rev. A, 236(12):149, 1997.

[84] S.V.G. Menon, V.K. Kelkar, and C. Manohar. Application of baxter's model to the cloud point of nonionic surfactant solutions. Phys. Rev. A, 43(2):1130, 1991.

[85] K.L. Mittal. Micellization, Solubilization and Microemulsions. Plenum Press, 1977.

[86] E. Moses, J. Fineberg, and V. Steinberg. Multistability and confined traveling-wave patterns in a convecting binary mixture. Phys. Rev. A, 35(6):2757, 1987.

[87] E. Moses and V. Steinberg. Competing patterns in a convective binary mixture. Phys. Rev. Lett., 57(16):2018, 1986.

[88] E. Moses and V. Steinberg. Flow patterns and nonlinear behavior of traveling waves in a convective binary fluid. Phys. Rev. A, 34(1):693, 1986.

[89] E. Moses and V. Steinberg. Stationary convection in a binary mixture. Phys. Rev. A, 43(2):707, 1991.

[90] R. Nagarajan and E. Ruckenstein. Molecular theory of microemulsions. Langmuir, 16:6400, 2000.

[91] E.W. (ed.) National Research Concil of the United States of America, Washburn. International critical tables of numerical data, physics, chemistry and technology. McGraw-Hill Book Company, Inc., New York, 1928.

[92] N.-T. Nguyen and S.T. Wereley. Fundamentals and applications of microfluidics. Artech House Publishers, 2006.

[93] J. Niederländer, M. Lücke, and M. Kamps. Weakly nonlinear convection: Galerkin model, numerical simulation, and amplitude equation. Zeits. Phys. B: Cond. Matt., 82:135, 1991.

[94] C. Normand, Y. Pomeau, and M.G. Velarde. Convective instability: A physicist's approach. Rev. Mod. Phys., 49:581, 1977.

[95] National Institute of Standards and USA Internet data base Technology. www.nist.gov, 2007. 
[96] A. Onuki. Phase transition of fluids in shear flow. J. Phys.: Cond. Matter, 9:6119, 1997.

[97] C. Pépin, T.K. Bose, and J. Thoen. Refractive index near the critical point and pressure dependence of the critical temperature of a watersodium di-2-sthylhexylsulfsucinat-decane microemulsion. Phys. Rev. A, 39(2):835, 1989.

[98] R. Piazza and A. Guarino. Soret effect in interacting micellar solutions. Phys. Rev. Lett., 88(20):208302-1, 2002.

[99] J.K. Platten and C.J. Legros. Convection in Liquids. Springer, 1984.

[100] J.K. Platten, D. Villers, and O. Lhost. LDV study of some free convection problems at extremely slow velocities: Soret driven convection and Marangoni convection. Instituto Superior Technico, Lisbon, Portugal, 1988.

[101] C.M. Pooley, O. Kukenok, and A.C. Balazs. Convection-driven pattern formation in phase-separating binary fluids. Phys. Rev. E, 71:030501(R), 2005.

[102] L.M. Prince. Microemulsions, Theory and Practice. Academic Press, 1977.

[103] M. Raffel, C. Willert, , S.T. Wereley, and J. Kompenhans. Particle Image Velocimetry, a practical guide. Springer, 2007.

[104] D. Ripple and R.F. Berg. Flow of microemulsions through microscopic pores. J. Chem. Phys., 97(10):7761, 1992.

[105] J. Rouch, A. Safouane, and P. Tartaglia. Static and dynamic light scattering studies of water-in-oil microemulsions in the critical region. evidence of a cross-over effect. J. Chem. Phys., 90(7):3756, 1989.

[106] E. Ruckenstein. Can phoretic motions be treated as interfacial tension gradient driven phenomena? J. Coll. Interf. Sci., 83(1):77, 1981.

[107] A. Ryskin, H.W. Müller, and H. Pleiner. Thermal convection in binary fluid mixtures with a weak concentration diffusivity but strong solutal forces. Phys. Rev. E, 67:046302-1, 2003.

[108] W. Schrader. Fluktuationen in Membranen - Schallgeschwindigkeitsmessungen, Kalorimetrie sowie dielektrische und akustische Spektroskopie an wässrigen Phospholipidsuspensionen (in german). PhD thesis, GeorgAugust-University of Göttingen, Germany, 2001.

[109] J.V. Sengers. Transport coefficients of fluids near critical points. Int. J. Thermophys., 6(3):203, 1985. 


\section{Bibliography}

[110] G.S. Settles. Schlieren and Shadowgraph Techniques. Springer, 2001.

[111] K. Shukla and R. Rjagopalan. Structure and phase transitions in colloidal dispersions from theory and simulation. Int. J. Thermophys., 16(2):327, 1995.

[112] L. Sirovich. The dynamics of coherent structures, I-III. Quart. Appl. Math., XLV(3):561, 1987.

[113] J. Timmermans. The physico-chemical constants of binary systems in concentrated solutions, Vol. 4, Systems with inorganic + organic or inorganic compounds (Excepting metallic derivatives). Interscience Publishers, 1960.

[114] J. Wang and M. Fiebig. Measurement of the thermal diffusivity of aqueous solutions of alcohols by a laser-induced thermal grating technique. Int. J. Thermophys., 16(6):1353, 1995.

[115] H. Watanabe and D.J. Seong. The thermal conductivity and thermal diffusivity of liquid n-alkanes: $C_{n} H_{2 n+2}(n=5$ to 10) and toluene. Int. J. Thermophys., 23(2):337, 2002.

[116] D.B. White. The planeforms and onset of convection with a temperaturedependent viscosity. J. Fluid Mech., 191:247, 1988.

[117] S. Wiegand. Thermal diffusion in liquid mixtures and polymer solutions. J. Phys.: Cond. Matter, 16:375, 2004.

[118] A. Xueqin, F. Jiao, and S. Weiguo. Critical behaviour of a pseudobinary system for a three-component microemulsion. J. Phys. Chem., 100:16674, 1996.

[119] J. Zierep and H. jr. Oertel, editors. Convective Transport an Instability Phenomena. G. Braun, Karlsruhe, 1982. 


\section{Appendix}

\section{.1 Physico-chemical parameters}

\section{$.1 .1 n$-Decane}

$$
\begin{aligned}
\text { density: } & \rho=726.44 \mathrm{Kg} / \mathrm{m}^{3}[95] \\
\text { dynamic viscosity: } & \mu=0.84696 \cdot 10^{-3} \mathrm{~Pa} \cdot \mathrm{s}[95] \\
& \mu(T)=8.65 \cdot 10^{-5} \cdot e^{1370 / T}, \\
& T=[273 \ldots 313][11] ; \\
\text { kinematic viscosity: } & \nu=1.1659 \cdot 10^{-6} \mathrm{~m}^{2} / \mathrm{s}[95] \\
\text { specific heat: } & c_{p}=2.1927 \cdot 10^{3} \mathrm{~J} / \mathrm{KgK}[95] \\
\text { thermal conductivity: } & \lambda=0.12944 \mathrm{~W} / \mathrm{mK}[95] \\
& \lambda(T)=0.13599(2) \\
& +2.568(06) \cdot 10^{4} \cdot(T-273.15), \\
& T=253 \ldots 343 \mathrm{~K}[115] \\
& \kappa=0.81262 \cdot 10^{-7} \mathrm{~m}{ }^{2} / \mathrm{s}[95] \\
& \kappa(T)=8.424(10) \cdot 10^{-8} \\
& +1.519(24) \cdot 10^{2} \cdot(T-273.15), \\
& T=253 \ldots 343 \mathrm{~K}[115] ; \\
\text { thermal diffusivity: } & \alpha=0.0011 / \mathrm{K}[95] ; \\
\text { thermal expansion: } & \operatorname{Pr}=14[95] .
\end{aligned}
$$

Table .1: Substance parameters of pure n-decane at $T=298 \mathrm{~K}$. 


\section{.1.2 Ethanol-Water Mixtures}

$$
\begin{aligned}
\text { density: } & \rho=931.68 \mathrm{Kg} / \mathrm{m}^{3}[91] \\
\text { dynamic viscosity: } & \mu=2.7328 \cdot 10^{-6} \mathrm{~Pa} \cdot \mathrm{s}[113] \\
\text { kinematic viscosity: } & \nu=2.499 \cdot 10^{-6} \mathrm{~m}^{2} / \mathrm{s} \mathrm{[113,} \mathrm{91]} \\
\text { thermal conductivity: } & \lambda=0.338 \mathrm{~W} / \mathrm{mK}[114] \\
\text { thermal diffusivity: } & \kappa=0.877 \cdot 10^{-7} \mathrm{~m}^{2} / \mathrm{s}[114] \\
\text { themal expansion: } & \alpha=0.80 \cdot 10^{-3} 1 / \mathrm{K}[113] \\
\text { concentration expansion: } & \beta=2.2 \cdot 10^{-3} 1 / \mathrm{K}[113] \\
\text { mass diffusion: } & D=3.99 \cdot 10^{-10} \mathrm{~m}^{2} / \mathrm{s}[63] \\
\text { Soret coefficient: } & S_{T}=-2.6 \cdot 10^{-3}[63] \\
\text { separation ratio: } & \psi=0.23[63] \\
\text { Lewis number: } & L e=4.5 \cdot 10^{-3} \\
\text { Prandtl number: } & \operatorname{Pr}=27
\end{aligned}
$$

Table .2: Thermodynamic properties of a $40 \% w t$ ethanol-water binary mixtures at $T=298 \mathrm{~K}$.

$$
\begin{aligned}
\text { density: } & \rho=957.0 \mathrm{Kg} / \mathrm{m}^{3}[91] \\
\text { dynamic viscosity: } & \mu=1.796 \cdot 10^{-6} \mathrm{~Pa} \cdot \mathrm{s}[113] \\
\text { kinematic viscosity: } & \nu=1.8767 \cdot 10^{-6} \mathrm{~m}^{2} / \mathrm{s}[113,91] \\
\text { thermal conductivity: } & \left.\lambda=0.440 \mathrm{~W} / \mathrm{mK}^{114}\right] \\
\text { thermal diffusivity: } & \kappa=1.07 \cdot 10^{-7} \mathrm{~m}^{2} / \mathrm{s}[114] \\
\text { themal expansion: } & \alpha=0.58 \cdot 10^{-3} 1 / \mathrm{K}[113] \\
\text { concentration expansion: } & \beta=1.6 \cdot 10^{-3} 1 / \mathrm{K}[113] \\
\text { mass diffusion: } & D=6.57 \cdot 10^{-10} \mathrm{~m}^{2} / \mathrm{s}[63] \\
\text { Soret coefficient: } & S_{T}=1.3 \cdot 10^{-3}[63] \\
\text { separation ratio: } & \psi=-0.087[63] \\
\text { Lewis number: } & \operatorname{Le}=6.1 \cdot 10^{-3} \\
\text { Prandtl number: } & \operatorname{Pr}=17
\end{aligned}
$$

Table .3: Thermodynamic properties of a $25 \% w t$ ethanol-water binary mixtures at $T=298 \mathrm{~K}$. 


\section{.1.3 The WDA Microemulsion}

$$
\begin{aligned}
\text { molar ratio: } & \omega 40.8 \\
\text { volume fraction: } & \eta_{c}=0.073[\underline{66}, \underline{67}, \\
& \eta_{c}=0.08[\underline{22]} \\
\text { critical temperature: } & T_{c}=36.2 \mathrm{~K} \mathrm{[66]}, \\
& T_{c} \simeq 43 \mathrm{~K}[\underline{68}, \\
& T_{c}=42.7 \mathrm{~K}[22]
\end{aligned}
$$

Table .4: Critical compositions and temperatures for the WDA system.

correlation length: coexistence curve:

$\xi=\xi_{0} \epsilon^{-\nu}, \xi_{0}=12 \AA, \nu=0.63[51]$

refractive index:

$$
\begin{aligned}
& \left(\eta^{(+)}-\eta^{(-)}\right)=1.025(4) \cdot \epsilon^{0.325(2)}-0.41(7) \cdot \epsilon^{0.325(2)+0.5}[118 \\
& n(T)-n\left(T_{c}\right)=\delta n_{c}+N_{2} \cdot \tau+N_{3} \cdot \tau^{1-\alpha} \text { 97] } \\
& \left(\delta n_{c}=O\left(10^{-8}\right), N_{2}=0.161(5), N_{3}=-0.011(2), \alpha=0.11\right) \\
& d T_{c} / d P=-14.3(2) \cdot 10^{-8}[97
\end{aligned}
$$

Table .5: Parameters and critical indices measured for the WDA system.

$$
\begin{aligned}
\text { density: } & \left.\rho(T[\mathrm{~K}]) / \mathrm{m}^{3}\right]=(0.73988(1)+0.0234(1) \cdot \eta) \cdot 10^{6} \text { [41] } \\
& \left(\epsilon=\mathrm{O}(1)-\mathrm{O}\left(10^{-3}\right)\right)
\end{aligned}
$$

dynamic viscosity: $\quad \mu(T)=\mu^{*} \cdot \tau^{-y}, y=0.030(3)$ [11]

Table .6: Temperature dependent properties of the critical WDA system. 
Appendix 


\section{Acknowledgements}

I am very much indebted to my advisor Professor Dr. A. Tilgner for accepting the subject I proposed and valuable discussion especially in the later stage of the work. I am grateful to Professor Dr. Dr. A. Dillmann who allowed me great latitude in working on a subject aside the main focus of DLR as well as I would like to thank the other theses commity members Professor Dr. W. Lauterborn, Professor Dr. U. Parlitz, Professor Dr. W. Glatzel, and Professor Dr. W. Kollatschny.

Since the subject of the work touches upon several different regions of physics I am indebted to quite a few people in many different ways. Particularly, I would like to thank Professor Dr. V. Steinberg for introducing me to the field of critical phenomena initiating this work during a very exiting and inspiring time I spent at the Weizmann Institute. Furthermore, I am grateful for discussion and encouragement by PD Dr. M. Rein and for the support by Dr. U. Kaatze concerning the DRS measurements. Finally, I would like to thank Professor Dr. M. Raffel for his continuous encouragement and support during the course of this work. 


\section{Lebenslauf}

Name:

geboren:

Staatsangehörigkeit:

Familienstand:

$1984-1988$

$1988-1990$

$1990-1997$

Juni 1997

$1997-1998$

$1998-2001$

$2001-2003$

05/2002-09/2003

November 2003

seit $02 / 2004$

08/2004-07/2005
Kolja Kindler

07.August 1977 in Bad Gandersheim

deutsch

ledig

Grundschule Bad Gandersheim

Orientierungsstufe Bad Gandersheim

Roswitha Gymnasium Bad Gandersheim

Abitur

Wehrdienst im Panzerbataillon 383 in Bad Frankenhausen

Physikstudium an der Ruprecht-Karls-Universität Heidelberg

Fortsetzung des Physikstudiums an der GeorgAugust-Universität Göttingen

Diplomarbeit am Institut für Aerodynamik und Strömungstechnik, Deutsches Zentrum für Luft und Raumfahrt, Göttingen unter Anleitung von Prof. Dr. D. Ronneberger. Titel der Arbeit: Kohärente Strukturen in kritischen Tragflügelströmungen

Diplomprüfung

Anstellung am Institut für Aerodynamik und Strömungstechnik, Deutsches Zentrum für Luft und Raumfahrt, Göttingen

Gaststudent in der Gruppe Nonlinear Physics and Fluid Dynamics, Department of Physics of Complex Systems, Weizmann Institute of Science in Rehovot, Israel 